\title{
SOLITON RESOLUTION FOR EQUIVARIANT WAVE MAPS ON A WORMHOLE: I
}

\author{
CASEY RODRIGUEZ
}

\begin{abstract}
Aвstract. In this paper, we initiate the study of finite energy equivariant wave maps from the $(1+3)$-dimensional spacetime $\mathbb{R} \times\left(\mathbb{R} \times \mathbb{S}^{2}\right) \rightarrow \mathbb{S}^{3}$ where the metric on $\mathbb{R} \times\left(\mathbb{R} \times \mathbb{S}^{2}\right)$ is given by

$$
d s^{2}=-d t^{2}+d r^{2}+\left(r^{2}+1\right)\left(d \theta^{2}+\sin ^{2} \theta d \varphi^{2}\right), \quad t, r \in \mathbb{R},(\theta, \varphi) \in \mathbb{S}^{2} .
$$

The constant time slices are each given by the Riemannian manifold $\mathcal{M}:=\mathbb{R} \times \mathbb{S}^{2}$ with metric

$$
d s^{2}=d r^{2}+\left(r^{2}+1\right)\left(d \theta^{2}+\sin ^{2} \theta d \varphi^{2}\right)
$$

The Riemannian manifold $\mathcal{M}$ contains two asymptotically Euclidean ends at $r \rightarrow \pm \infty$ that are connected by a spherical throat of area $4 \pi^{2}$ at $r=0$. The spacetime $\mathbb{R} \times \mathcal{M}$ is a simple example of a wormhole geometry in general relativity. In this work we will consider 1-equivariant or corotational wave maps. Each corotational wave map can be indexed by its topological degree $n$. For each $n$, there exists a unique energy minimizing corotational harmonic map $Q_{n}: \mathcal{M} \rightarrow \mathbb{S}^{3}$ of degree $n$. In this work, we show that modulo a free radiation term, every corotational wave map of degree $n$ converges strongly to $Q_{n}$. This resolves a conjecture made by Bizon and Kahl in [3] in the corotational case.
\end{abstract}

\section{Introduction}

There has been an increased interest in recent years in the study of geometric nonlinear wave equations. One of the fundamental models considered is the following wave map model. Let $(M, g)$ be a $(1+d)-$ dimensional Lorentzian spacetime, and let $(N, h)$ be a Riemannian manifold. A wave map $U: M \rightarrow N$ is a formal critical point of the action functional

$$
\mathcal{S}(U, \partial U)=\frac{1}{2} \int_{M} g^{\mu v}\left\langle\partial_{\mu} U, \partial_{\nu} U\right\rangle_{h} d g .
$$

In local coordinates, the Euler-Lagrange equations associated to $\mathcal{S}$ is the following system of semilinear wave equations

$$
\square_{g} U^{i}+\Gamma_{j k}^{i}(U) \partial_{\mu} U^{j} \partial_{\nu} U^{k} g^{\mu v}=0,
$$

where $\square_{g}:=\frac{1}{\sqrt{-g}} \partial_{\mu}\left(g^{\mu v} \sqrt{-g} \partial_{v}\right)$ is the wave operator associated to the background spacetime $(M, g)$ and $\Gamma_{j k}^{i}$ are the Christoffel symbols associated to the target $(N, h)$. The system is collectively referred to as the wave map system and is also known in the physics literature as the classical nonlinear $\sigma$-model. A particular case that has been intensely studied is the case when $M$ is $(1+d)$-dimensional Minkowski space $\mathbb{R}^{1+d}$ with the flat metric (see the classical reference [25] and the recent review [22]). From a mathematical point of view, a wave map $U: \mathbb{R}^{1+d} \rightarrow N$ can be considered as a geometric generalization of the free wave equation on Minkowski space. Indeed, if we take $N=\mathbb{R}$ with, then the wave map equations (1.2) reduce to the free wave equation on Minkowski space

$$
\partial_{t}^{2} U-\Delta U=0, \quad(t, x) \in \mathbb{R}^{1+d} .
$$

From a physical point of view, wave maps $U: \mathbb{R}^{1+3} \rightarrow \mathbb{S}^{3}$ describe fields which approximate a low energy regime of QCD (see [8] and [9] for nice introductions to this perspective).

The case of a curved spacetime is relatively unexplored. In this work, we study corotational wave maps on a curved background. In particular, we consider wave maps $U: \mathbb{R} \times\left(\mathbb{R} \times \mathbb{S}^{2}\right) \rightarrow \mathbb{S}^{3}$ where the background metric is given by

$$
d s^{2}=-d t^{2}+d r^{2}+\left(r^{2}+1\right)\left(d \theta^{2}+\sin ^{2} \theta d \varphi^{2}\right), \quad t, r \in \mathbb{R},(\theta, \varphi) \in \mathbb{S}^{2} .
$$


The constant time slices correspond to the Riemannian manifold $\mathcal{M}:=\mathbb{R} \times \mathbb{S}^{2}$ with metric

$$
d s^{2}=d r^{2}+\left(r^{2}+1\right)\left(d \theta^{2}+\sin ^{2} \theta d \varphi^{2}\right), \quad r \in \mathbb{R},(\theta, \varphi) \in \mathbb{S}^{2} .
$$

Heuristically, $\mathcal{M}$ has two asymptotically Euclidean ends at $r= \pm \infty$ connected by a spherical throat at $r=0$. In the general relativity literature, the spacetime $\mathbb{R} \times \mathcal{M}$ is a simple example of a 'wormhole geometry.' $\mathrm{A}$ corotational wave map $U: \mathbb{R} \times \mathcal{M} \rightarrow \mathbb{S}^{3}$ is given by the ansatz

$$
U(t, r, \theta, \varphi)=(\psi(t, r), \theta, \varphi) \in \mathbb{S}^{3},
$$

where $\psi$ is the azimuth angle on $\mathbb{S}^{3}$. For $U$ given by (1.5), the action $\mathcal{S}$ given by (1.1) reduces to

$$
\mathcal{S}(\psi, \partial \psi)=\frac{1}{2} \int_{\mathbb{R}} \int_{\mathbb{R}}\left[-\left|\partial_{t} \psi(t, r)\right|^{2}+\left|\partial_{r} \psi(t, r)\right|^{2}+\frac{2 \sin ^{2} \psi(t, r)}{r^{2}+1}\right]\left(r^{2}+1\right) d r d t,
$$

and the wave map equation (1.2) reduces to the single semilinear wave equation

$$
\begin{aligned}
& \partial_{t}^{2} \psi-\partial_{r}^{2} \psi-\frac{2 r}{r^{2}+1} \partial_{r} \psi+\frac{\sin 2 \psi}{r^{2}+1}=0, \quad(t, r) \in \mathbb{R} \times \mathbb{R}, \\
& \vec{\psi}(0)=\left(\psi_{0}, \psi_{1}\right) .
\end{aligned}
$$

Here we use the notation $\vec{\psi}(t)=\left(\psi(t, \cdot), \partial_{t} \psi(t, \cdot)\right)$. In this work, solutions $\psi$ to (1.6) will be referred to as corotational wave maps on a wormhole. The equation (1.6) has the following conserved energy along the flow:

$$
\mathcal{E}(\vec{\psi}(t)):=\frac{1}{2} \int_{\mathbb{R}}\left[\left|\partial_{t} \psi(t, r)\right|^{2}+\left|\partial_{r} \psi(t, r)\right|^{2}+\frac{2 \sin ^{2} \psi(t, r)}{r^{2}+1}\right]\left(r^{2}+1\right) d r=\mathcal{E}(\vec{\psi}(0)) .
$$

In order for the initial data to have finite energy, we must have for some $m, n \in \mathbb{Z}$,

$$
\psi_{0}(-\infty)=m \pi \quad \text { and } \quad \psi_{0}(\infty)=n \pi .
$$

For a finite energy solution $\vec{\psi}(t)$ to $(1.6)$ to depend continuously on $t$, we must have that $\psi(t,-\infty)=m \pi$ and $\psi(t, \infty)=n \pi$ for all $t$. In this work, we will, without loss of generality, fix $m=0$ and assume $n \in \mathbb{N} \cup\{0\}$. Thus, we only consider wave maps which send the left Euclidean end at $r=-\infty$ to the north pole of $\mathbb{S}^{3}$. The integer $n$ is referred to as the topological degree of the map $\psi$ and, heuristically, represents the number of times $\mathcal{M}$ gets wrapped around $\mathbb{S}^{3}$ by $\psi$. For each $n \in \mathbb{N} \cup\{0\}$, we denote the space of finite energy pairs of degree $n$ by

$$
\mathcal{E}_{n}:=\left\{\left(\psi_{0}, \psi_{1}\right): \mathcal{E}\left(\psi_{0}, \psi_{1}\right)<\infty, \quad \psi_{0}(-\infty)=0, \quad \psi_{0}(\infty)=n \pi\right\} .
$$

In this work, we classify the long time dynamics of all finite energy corotational wave maps on a wormhole.

There has been widespread belief in the mathematical physics community that for most globally wellposed dispersive equations, a solution asymptotically decouples into a coherent element and a purely dispersive element. The coherent element is nonlinear in nature and is determined by the static solutions and symmetries of the equation (i.e. solitons). The purely dispersive element is a solution to the underlying linear equation. This heuristic belief goes by the name of the soliton resolution conjecture. There are features that wave maps on a wormhole exhibit that make it an interesting model in which to study this phenomenon. The first feature is that showing global well-posedness, i.e. every solution $\vec{\psi}(t)$ to (1.6) exists for all $t \in \mathbb{R}$, is simple. The geometry of the domain removes the possibility of singularity formation at the origin and renders the equation essentially energy-subcritical. Another feature of this model is the abundance of finite energy static solutions to (1.6) which also go by the name of harmonic maps. In particular, it can be shown that for every $n \in \mathbb{N} \cup\{0\}$, there exists a unique solution $Q_{n}$ to the static equation

$$
\begin{aligned}
& \partial_{r}^{2} F+\frac{2 r}{r^{2}+1} \partial_{r} F-\frac{\sin 2 F}{r^{2}+1}=0, \\
& F(-\infty)=0, \quad F(\infty)=n \pi,
\end{aligned}
$$

and $Q_{n}$ has finite energy (see Section 2). Moreover, each $Q_{n}$ is linear stable (see Section 5). In [3], Bizon and Kahl gave numerical evidence for the following soliton resolution conjecture for this model: for every 
$n \in \mathbb{N} \cup\{0\}$ and for any $\left(\psi_{0}, \psi_{1}\right) \in \mathcal{E}_{n}$, there exist a unique globally defined solution $\psi$ to (1.6) and solutions $\varphi_{L}^{ \pm}$to the underlying linear equation

$$
\partial_{t}^{2} \varphi-\partial_{r}^{2} \varphi-\frac{2 r}{r^{2}+1} \partial_{r} \varphi+\frac{2}{r^{2}+1} \varphi=0,
$$

such that

$$
\vec{\psi}(t)=\left(Q_{n}, 0\right)+\vec{\varphi}_{L}^{ \pm}(t)+o(1),
$$

as $t \rightarrow \pm \infty$. In this work we verify this conjecture. As alluded to in the initial description of the background $\mathbb{R} \times \mathcal{M}$, the spacetime $\mathbb{R} \times \mathcal{M}$ and Riemannian manifold $\mathcal{M}$ have appeared in contexts outside of this work. For example, $\mathbb{R} \times \mathcal{M}$ has been considered in the general relativity as a prototype geometry representing a wormhole since it was first introduced by Ellis in the 1970's and later popularized by Morris and Thorne in the 1980's (cf. [20] and [7] and the references therein). Also, the two dimensional version of $\mathcal{M}$ given by $\mathcal{M}^{2}=\mathbb{R} \times \mathbb{S}^{1}$ with metric

$$
d s^{2}=d r^{2}+\left(r^{2}+1\right) d \varphi^{2},
$$

is simply an intrinsic description of the classical catenoid surface.

We now turn to stating our main result. In what follows we use the following notation. If $r_{0} \geq-\infty$ and $w(r)$ is a positive continuous function on $\left[r_{0}, \infty\right)$, then we define

$$
\left\|\left(\psi_{0}, \psi_{1}\right)\right\|_{\mathcal{H}\left(\left[r_{0}, \infty\right) ; w(r) d r\right)}^{2}:=\int_{r_{0}}^{\infty}\left[\left|\psi_{0}(r)\right|^{2}+\left|\psi_{1}(r)\right|^{2} d r\right] w(r) d r
$$

The Hilbert space $\mathcal{H}\left(\left[r_{0}, \infty\right) ; w(r) d r\right)$ is then defined to be the completion of pairs of smooth functions with compact support in $\left(r_{0}, \infty\right)$ under the norm previously defined. Let $n \in \mathbb{N} \cup\{0\}$ be a fixed topological degree. In the $n=0$ case, the natural space to place the solution $\vec{\psi}(t)$ to (1.6) in is the energy space $\mathcal{H}_{0}:=\mathcal{H}\left((-\infty, \infty) ;\left(r^{2}+1\right) d r\right)$. Indeed, it is easy to show that $\|\vec{\psi}\|_{\mathcal{E}_{0}} \simeq\|\vec{\psi}\|_{\mathcal{H}_{0}}$. For $n \geq 1$, we measure distance relative to $\left(Q_{n}, 0\right)$ and define $\mathcal{H}_{n}:=\mathcal{E}_{n}-\left(Q_{n}, 0\right)$ with 'norm'

$$
\|\vec{\psi}\|_{\mathcal{H}_{n}}:=\left\|\vec{\psi}-\left(Q_{n}, 0\right)\right\|_{\mathcal{H}_{0}} \text {. }
$$

Note that $\psi(r)-Q_{n}(r) \rightarrow 0$ as $r \rightarrow \pm \infty$. The main result of this work is the following.

Theorem 1.1. For any energy data $\left(\psi_{0}, \psi_{1}\right) \in \mathcal{E}_{n}$, there exists a unique globally defined solution $\vec{\psi}(t) \in C\left(\mathbb{R} ; \mathcal{H}_{n}\right)$ which scatters forwards and backwards in time to the harmonic map $\left(Q_{n}, 0\right)$, i.e. there exist solutions $\varphi_{L}^{ \pm}$to the linear equation (1.8) such that

$$
\vec{\psi}(t)=\left(Q_{n}, 0\right)+\vec{\varphi}_{L}^{ \pm}(t)+o_{\mathcal{H}_{0}}(1)
$$

as $t \rightarrow \pm \infty$.

We remark that in [3] Bizon and Kahl gave numerical evidence that soliton resolution holds in the more general $\ell$-equivariant setting (here corotational corresponds to $\ell=1$ ). In the companion work [21] we prove this and completely resolve the soliton resolution conjecture for all equivariant wave maps on a wormhole.

We point out that an equation with properties similar to the model considered in this paper was studied in [18], [14], and [16] and served as a road map for the work carried out here. In these works, the authors studied $\ell$-equivariant wave maps $U: \mathbb{R} \times(\mathbb{R} \backslash B(0,1)) \rightarrow \mathbb{S}^{3}$ such that $U(\partial B(0,1))=\{(0,0,0,1)\}$. An $\ell$-equivariant wave map $U$ is determined by the associated azimuth angle $\psi(t, r)$ which satisfies the equation

$$
\begin{aligned}
& \partial_{t}^{2} \psi-\partial_{r}^{2} \psi-\frac{2}{r} \partial_{r} \psi+\frac{\ell(\ell+1)}{2\left(r^{2}+1\right)} \sin 2 \psi=0, \quad t \in \mathbb{R}, r \geq 1, \\
& \psi(t, 1)=0, \quad \psi(t, \infty)=n \pi, \quad \forall t .
\end{aligned}
$$

Such wave maps were called $\ell$-equivariant exterior wave maps. Similar to wave maps on a wormhole, global well-posedness and an abundance of harmonic maps hold for the exterior wave map equation (1.9). In the works [18], [14], and [16], the authors proved the soliton resolution conjecture for $\ell$-equivariant exterior wave maps for arbitrary $\ell \geq 1$. However, the geometry of the background $\mathbb{R} \times(\mathbb{R} \backslash B(0,1))$ is still flat and could be considered artificial. On the other hand, the wormhole geometry considered in this work contains curvature which make wave maps on a wormhole more geometric in nature while still retaining 
the properties that make them ideal for studying the soliton resolution conjecture. We remark here that, to the author's knowledge, Theorem 1.1 is the first result that establishes the soliton resolution conjecture for arbitrary corotational finite energy wave maps on a curved background. See [17] for soliton resolution for corotational wave maps from $\mathbb{R} \times \mathbb{H}^{2} \rightarrow \mathbb{H}^{2}$ with a restriction on the behavior at infinity.

The method of proof used in the works [18], [14], and [16] to establish the soliton resolution conjecture for (1.9) was the celebrated concentration-compactness/rigidity theorem method pioneered by Kenig and Merle in [12] and [13]. In [14] and [16], the authors used a 'channels of energy' argument based on exterior energy estimates for free waves on $\mathbb{R}^{1+d}$ with $d$ odd to close the argument (see [5] and [15] for these estimates). The proof of our main result, Theorem 1.1, uses a similar methodology which we now briefly overview. The proof is by contradiction and is split into three main steps. In the first step, we establish a small data theory for (1.6), i.e. if $\|\vec{\psi}(0)\|_{\mathcal{H}_{n}}$ is sufficiently small, then the solution $\psi$ to (1.6) is global and scatters to $\left(Q_{n}, 0\right)$. In the second step, using concentration-compactness arguments and the first step we show that if Theorem 1.1 fails, then there exists a solution $\vec{\psi}_{*} \neq\left(Q_{n}, 0\right)$ to (1.6) such that the trajectory $\left\{\vec{\psi}_{*}(t): t \in \mathbb{R}\right\}$ is precompact in $\mathcal{H}_{n}$. In the third and final step, we establish the following rigidity theorem: if $\psi$ is a solution to (1.6) such that $\{\vec{\psi}(t): t \in \mathbb{R}\}$ is precompact in $\mathcal{H}_{n}$, then $\vec{\psi}=\left(Q_{n}, 0\right)$. This rigidity theorem contradicts the second step which implies that Theorem 1.1 must hold.

We now give an outline of the paper and provide a few more details of the previously sketched steps. Section 2, Section 3, and Section 4 contain preliminaries necessary to carry out the concentration-compactness/ rigidity theorem methodology for wave maps on a wormhole. In Section 2, we establish various properties of the harmonic maps $Q_{n}$ needed throughout the work. In particular, we establish existence, uniqueness, and asymptotics. Establishing these properties in the exterior wave map model is considerably simpler since the static solutions to (1.9) (in the corotational case) are governed by the well-known equation for a damped pendulum

$$
\frac{d^{2} F}{d x^{2}}+\frac{d F}{d x}=\sin 2 F, \quad x=\log r
$$

The properties needed can then be derived from a simple phase plane analysis. However, in our setting there is no such change of variables that renders (1.7) autonomous. We instead use classical ODE arguments inspired by the work on corotational Skyrmions [19] to derive the properties we need. In Section 3 and Section 4, we establish results needed to carry out the first two steps in the concentration-compactness /rigidity theorem methodology. We first reformulate Theorem 1.1 as the statement that all radial solutions to a certain semilinear wave equation of the form

$$
\partial_{t}^{2} u-\Delta_{g} u+V(r) u=N(r, u), \quad(t, r) \in \mathbb{R} \times \mathbb{R} .
$$

scatter to free waves as $t \rightarrow \pm \infty$ (see Theorem 4.1 for the exact statement). Here $u$ is related to $\psi$ by $u=\frac{1}{\left(r^{2}+1\right)^{1 / 2}}\left(\psi-Q_{n}\right), V(r)$ is a smooth potential arising from linearizing about $Q_{n}$, and $-\Delta_{g}$ is the Laplace operator on the $5 d$ wormhole $\mathcal{M}^{5}=\mathbb{R} \times \mathbb{S}^{4}$ with metric

$$
d s^{2}=d r^{2}+\left(r^{2}+1\right) d \Omega_{\mathbb{S}^{4}}^{2}
$$

where $d \Omega_{\mathbb{S}^{4}}^{2}$ is the round metric on the sphere $\mathbb{S}^{4}$. In the remainder of the paper we carry out the concentration-compactness/ rigidity theorem method in the equivalent ' $u$-formulation.' Establishing the first two steps in the $u$-formulation follows from fairly standard arguments once Strichartz estimates for radial solutions to the free wave equation $\partial_{t}^{2} u-\Delta_{g} u=0$ are established. In the exterior wave map model, these estimates follow from previously known results on Strichartz estimates for free waves on Riemannian manifolds. However, Strichartz estimates for free waves on a wormhole fall outside of the literature devoted to free waves on Riemannian manifolds because of the trapping that occurs at the throat $r=0$. In the works [23] and [24], the authors established dispersive estimates in geometries with trapping which are asymptotic to wormholes as $r \rightarrow \pm \infty$ as long as the initial data is localized to a fixed spherical harmonic (i.e. angular momentum). Since we are only interested in radial free waves on a wormhole, in Section 3 we are able to refine the dispersive estimates from [23] and [24] in the zero angular momentum case and obtain the Strichartz estimates we need. In fact, we establish Strichartz estimates for radial free waves on $d$-dimensional wormholes for arbitrary $d \geq 3$. This section is independent of all other sections and may be of interest in its own right. In Section 4, we make the reduction previously described and transfer the 
Strichartz estimates established in Section 3 for $\partial_{t}^{2}-\Delta_{g}$ to the perturbed operator $\partial_{t}^{2}-\Delta_{g}+V$. The fact that the Strichartz estimates for the free wave operator carry over to the perturbed operator hinges on spectral information for the Schrödinger operator $-\Delta_{g}+V$. In Section 5 and Section 6, we use the concentrationcompactness/rigidity theorem method to prove our main result. In Section 5 we carry out the first two steps of the concentration-compactness/rigidity theorem methodology in the $u$-formulation. The main result of this section is that if Theorem 1.1 fails so that not all solutions to (1.10) scatter, then there exists a nonzero solution $u_{*}$ to (1.10) such that $\left\{\vec{u}_{*}(t): t \in \mathbb{R}\right\}$ is precompact in $\mathcal{H}:=\mathcal{H}\left((-\infty, \infty) ;\left(r^{2}+1\right)^{2} d r\right)$. In Section 6, we show that a solution $u$ to (1.10) such that $\{\vec{u}(t): t \in \mathbb{R}\}$ is precompact in $\mathcal{H}$ must be identically 0 which completes the proof. In particular, we show that $u$ is zero by showing it must be a static solution to (1.10) with finite energy. This is achieved using a change of variables valid in the exterior regions $|r| \gtrsim 1$ that transforms (1.10) into an 'exterior wave map equation'. We then use channels of energy arguments similar to those used in [14] and [16] to show that $u$ is a static solution to (1.10). This then implies that $\psi=Q_{n}+\left(r^{2}+1\right)^{1 / 2} u$ satisfies (1.7). By the uniqueness of harmonic maps, we deduce that $u \equiv 0$ and conclude the proof.

Acknowledgments: This work was completed during the authors doctoral studies at the University of Chicago. The author would like to thank his adviser, Carlos Kenig, for his invaluable guidance and careful reading of the original manuscript. The author would also like to thank Wilhelm Schlag, Andrew Lawrie, and Piotr Bizon for helpful discussions and encouragement during the completion of this work.

\section{Harmonic Maps}

For the remainder of the paper, we fix a topological degree $n \in \mathbb{N} \cup\{0\}$. In this section, we study static solutions to (1.6). In particular, we prove the following.

Proposition 2.1. There exists a unique smooth solution $Q_{n}$ to the equation

$$
\begin{aligned}
& F^{\prime \prime}+\frac{2 r}{r^{2}+1} F^{\prime}-\frac{\sin 2 F}{r^{2}+1}=0, \quad r \in \mathbb{R}, \\
& F(-\infty)=0, \quad F(\infty)=n \pi .
\end{aligned}
$$

In the case $n=0, Q_{0}=0$. For $n \in \mathbb{N}, Q_{n}$ is increasing on $\mathbb{R}$, satisfies $Q(r)+Q(-r)=n \pi$ for all $r$ and there exists $\alpha_{n}>0$ such that,

$$
\begin{aligned}
& Q_{n}(r)=n \pi-\alpha_{n} r^{-2}+O\left(r^{-4}\right), \quad \text { as } r \rightarrow \infty, \\
& Q_{n}(r)=\alpha_{n} r^{-2}+O\left(r^{-4}\right), \quad \text { as } r \rightarrow-\infty .
\end{aligned}
$$

The $O(\cdot)$ terms also satisfy the natural derivative bounds.

The proof of existence follows from a simple shooting argument sketched in [3]. The proof of uniqueness and properties needed are inspired by the work on the equivariant Skyrme equation [19]. The proof of Proposition 2.1 will be contained in the following various lemmas.

2.1. Existence of Harmonic Maps. In this section we prove the existence part of Proposition 2.1 In order to achieve this and, in fact, uniqueness of the harmonic map constructed, we will need to study general solutions to

$$
F^{\prime \prime}+\frac{2 r}{r^{2}+1} F^{\prime}-\frac{\sin 2 F}{r^{2}+1}=0, \quad r \in \mathbb{R} .
$$

We begin with the following simple lemma.

Lemma 2.2. If $F$ is a solution to (2.1), then $F$ exists on all of $\mathbb{R}$. Moreover, $F$ has limits at $\pm \infty$ in $\mathbb{Z} \pi \cup\left(\mathbb{Z}+\frac{1}{2}\right) \pi$.

Proof. Suppose that $F$ solves (2.1). Due to the sublinear growth in $F, F^{\prime}$ in (2.1), it follows from standard ODE theory that $F$ is globally defined. Because of the invariance of the equation under the change $r \leftrightarrow-r$, we need only show that $F$ has a limit at $\infty$.

Define the following auxiliary function

$$
Q(r)=\left(r^{2}+1\right) \frac{\left(F^{\prime}\right)^{2}}{2}-\sin ^{2} F
$$


Using that $F$ solves (2.1), we have that

$$
Q^{\prime}(r)=-r\left(F^{\prime}\right)^{2}
$$

Thus, $Q$ is nonincreasing on $r \geq 0$ and by definition is also bounded below. Thus, $Q(r) \rightarrow c \in[-1, \infty)$ as $r \rightarrow \infty$. Moreover, we note that

$$
\left(\left(r^{2}+1\right) Q\right)^{\prime}=-2 r \sin ^{2} F \leq 0,
$$

so that

$$
Q(r) \leq \frac{Q(0)}{r^{2}+1}, \quad r \geq 0
$$

This implies $c \leq 0$.

The previous bound on $Q$ implies that

$$
F^{\prime}(r)^{2}=\frac{Q(r)}{r^{2}+1}+\frac{\sin ^{2} F(r)}{r^{2}+1} \sin ^{2} F(r)=O\left(\frac{1}{r^{2}}\right) .
$$

We now claim that $F^{\prime}$ isn't just $O\left(r^{-1}\right)$ but in fact satisfies

$$
F^{\prime}(r)=o\left(\frac{1}{r}\right)
$$

Suppose towards a contradiction that this is not the case. Then there exist $\delta>0$ and a sequence $r_{n} \rightarrow \infty$ with the property

$$
\frac{\delta}{r_{n}} \leq\left|F^{\prime}\left(r_{n}\right)\right|
$$

Since $F$ solves (2.1), we have that

$$
\left|F^{\prime \prime}(r)\right| \leq \frac{K}{r^{2}}
$$

Thus, for $r_{n} \leq r \leq(1+\delta / 2 K) r_{n}$, we have

$$
\left|F^{\prime}(r)-F^{\prime}\left(r_{n}\right)\right| \leq K \int_{r_{n}}^{r} \rho^{-2} d \rho \leq K\left(\frac{1}{r_{n}}-\frac{1}{r}\right) \leq \frac{\delta}{2 r_{n}},
$$

so that

$$
\left|F^{\prime}(r)\right| \geq \frac{\delta}{2 r_{n}}, \quad r_{n} \leq r \leq(1+\delta / 2 K) r_{n}
$$

Hence

$$
-Q^{\prime}(r)=r F^{\prime}(r)^{2} \geq \frac{\delta^{2}}{4 r_{n}}, \quad r_{n} \leq r \leq(1+\delta / 2 K) r_{n} .
$$

The previous estimate implies that

$$
\left|Q\left(r_{n}\right)-Q\left((1+\delta / 2 K) r_{n}\right)\right|=\int_{r_{n}}^{(1+\delta / 2 K) r_{n}}-Q(r) d r \geq \frac{\delta^{3}}{8 K^{\prime}}
$$

which contradicts the fact that $\lim _{r \rightarrow \infty} Q(r)$ exists in $[-1,0]$. Thus, the claim $F^{\prime}(r)=o\left(r^{-1}\right)$ holds.

We now show that as $r \rightarrow \infty, F(r)$ tends to $k \pi$ or $\left(k+\frac{1}{2}\right) \pi$ for some $k \in \mathbb{Z}$. Since $F^{\prime}(r)=o\left(r^{-1}\right)$ and $Q(r) \rightarrow c \in[-1,0]$, we have that

$$
\sin ^{2} F(r) \rightarrow \tilde{c} \in[0,1], \quad r \rightarrow \infty .
$$

Thus, $F(r)$ tends to some limit $F_{\infty} \in \mathbb{R}$ as $r \rightarrow \infty$. Since $F$ solves (2.1) and satisfies $F^{\prime}(r)=o\left(r^{-1}\right)$, we see that

$$
\left(r^{2}+1\right) F^{\prime \prime}(r) \rightarrow \sin 2 F_{\infty}, \quad \text { as } r \rightarrow \infty .
$$

If $\sin 2 F_{\infty} \neq 0$, then for large $r$ we have

$$
F^{\prime}(r)=\int_{r}^{\infty} F^{\prime \prime}(\rho) d \rho \sim \sin 2 F_{\infty} \int_{r}^{\infty} \frac{1}{\rho^{2}+1} d \rho \sim \sin 2 F_{\infty} \frac{1}{r},
$$

which contradicts $F^{\prime}(r)=o\left(r^{-1}\right)$. Thus, we must have that $\sin 2 F_{\infty}=0$ as desired. 
We remark that we will now be interested solely in solutions to (2.1) which satisfy $F( \pm \infty) \in \mathbb{Z} \pi$. This is because these solutions are the only solutions that have the potential to have finite energy $\mathcal{E}(F, 0)<\infty$. Using Lemma2.2 we can establish the following asymptotics for solutions to 2.1.).

Lemma 2.3. Suppose that $F$ solves (2.1) and there exists $k \in \mathbb{N} \cup\{0\}$ such that $F(\infty)=k \pi$. Then there exists $\alpha \in \mathbb{R}$ such that

$$
F(r)=k \pi+\alpha r^{-2}+O\left(r^{-4}\right),
$$

as $r \rightarrow \infty$ where the $O(\cdot)$ term satisfies the natural derivative bounds. A similar statement holds as $r \rightarrow-\infty$ if $F(-\infty)=k \pi$.

We note that Lemma 2.3 provides the asymptotics stated in Proposition 2.1

Proof. The proof of Lemma2.3 follows in almost exactly the same way as the proof of Case 1 of Theorem 2.3 in [19]. The idea is to make the change of variables $x=\operatorname{arcsinh} r$ and use the fact that $d F / d x=r d F / d r=o(1)$ to write (2.1) as

$$
\frac{d^{2} F}{d x^{2}}+\frac{d F}{d x}-\sin 2 F+O\left(e^{-2 x}\right)=0 .
$$

The ODE (2.4) is asymptotically the autonomous ODE $F^{\prime \prime}+F^{\prime}-\sin 2 F=0$ (the damped pendulum) near $x=\infty$ for which the desired expansion (2.3) holds in the $x$ variable. We omit the details and refer the reader to the proof of Case 1 in Theorem 2.3 in [19] for the full details of the argument.

A fact that will be useful in Section 6 is that one can obtain a solution to (2.1) with prescribed asymptotics as $r \rightarrow \infty$.

Proposition 2.4. Let $k \in \mathbb{N} \cup\{0\}$, and let $\alpha \in \mathbb{R}$. Then there exists a unique solution $F_{\alpha}$ to (2.1) such that

$$
F_{\alpha}(r)=k \pi+\alpha r^{-2}+O\left(r^{-4}\right)
$$

as $r \rightarrow \infty$ where the $O(\cdot)$ term satisfies the natural derivative bounds.

Before giving the proof, we note that the symmetry $r \mapsto-r$ of (2.1) allows us to conclude from Proposition 2.4 that given $k \in \mathbb{N} \cup\{0\}$ and $\beta \in \mathbb{R}$, there exists a solution $F_{\beta}(r)$ to (2.1) such that

$$
F_{\beta}(r)=k \pi+\beta r^{-2}+O\left(r^{-4}\right)
$$

as $r \rightarrow-\infty$.

Proof. We seek a solution $F$ to (2.1) with the stated asymptotics (2.5). We first make the change of variables $x=\operatorname{arcsinh} r$ so that (2.1) becomes

$$
F^{\prime \prime}+\tanh x F^{\prime}-\sin 2 F=0, \quad x \in \mathbb{R},
$$

where $F^{\prime}=\frac{d F}{d x}$. We now rewrite (2.6) as

$$
F^{\prime \prime}+F^{\prime}-2 F=[\sin 2 F-2 F]+(1-\tanh x) F^{\prime} .
$$

Define $G=e^{x / 2}(F-k \pi)$. Then $G$ satisfies

$$
G^{\prime \prime}+\frac{9}{4} G=N\left(x, G, G^{\prime}\right),
$$

where

$$
N\left(x, G, G^{\prime}\right)=e^{x / 2}\left[\sin \left(2 e^{-x / 2} G\right)-2 e^{-x / 2} G\right]+(1-\tanh x)\left[G^{\prime}-\frac{1}{2} G\right] .
$$

A fundamental system to the underlying linear equation $G^{\prime \prime}-\frac{9}{4} G=0$ is given by

$$
G_{1}(x)=e^{-3 x / 2}, \quad G_{2}(x)=e^{3 x / 2} .
$$

The Wronskian $W\left(G_{1}, G_{2}\right)=G_{1}^{\prime} G_{2}-G_{1} G_{2}^{\prime}$ is given by -3 . By the variation of constants formula, we seek a solution $G=G_{\alpha}$ to the integral equation

$$
G=\alpha G_{1}(x)+\frac{1}{3} \int_{x}^{\infty}\left[G_{1}(x) G_{2}(y)-G_{1}(y) G_{2}(x)\right] N\left(y, G, G^{\prime}\right) d y,
$$


for $x \geq R$ for some $R$. For $R>0$, define the Banach space $X_{R}=\left\{G \in C^{1}([R, \infty)):\|G\|_{X_{R}}<\infty\right\}$ where

$$
\|G\|_{X_{R}}:=\sup _{x \geq R} e^{3 x / 2}\left[|G(x)|+\left|G^{\prime}(x)\right|\right] .
$$

Denote the right side of (2.10) by $\Phi(G)$. From (2.9), it is easy to see that

$$
\left|N\left(y, G, G^{\prime}\right)\right| \leq e^{-y}|G|^{3}+e^{-2 y}\left[|G|+\left|G^{\prime}\right|\right] .
$$

Thus,

$$
\|\Phi(G)\|_{X_{R}} \leq|\alpha|+3|\alpha| / 2+C\left[e^{-R}\|G\|_{X_{R}}^{3}+e^{-2 R}\|G\|_{X_{R}}\right]
$$

For $R$ sufficiently large, a fixed point argument yields the existence of a unique solution $G_{\alpha}$ to (2.10). Moreover, $G_{\alpha}$ satisfies

$$
G_{\alpha}(x)=\alpha e^{-3 x / 2}+O\left(e^{-7 x / 2}\right)
$$

as $x \rightarrow \infty$. This means $F_{\alpha}(x)=k \pi+e^{-x / 2} G_{\alpha}(x)$ satisfies (2.6) and

$$
F_{\alpha}(x)=k \pi+\alpha e^{-2 x}+O\left(e^{-4 x}\right)
$$

as $x \rightarrow \infty$. This is the same as (2.5) under the change of variables $r=\sinh x$. This concludes the proof of existence of $F_{\alpha}$. Uniqueness follows from the fixed point argument and Lemma 2.3.

Using Lemma 2.3 and monotonicity of the auxiliary function $Q(r)$, we deduce the following monotonicity result for solutions to (2.1).

Lemma 2.5. Suppose that $F$ solves (2.1) and

$$
F(-\infty)=l \pi, \quad F(\infty)=k \pi .
$$

Then $F$ is monotonic on $\mathbb{R}$. In particular, if $l=k$, then $F$ is the constant solution.

Proof. Recall from the proof of Lemma 2.2 that the function

$$
Q(r)=\left(r^{2}+1\right) \frac{\left(F^{\prime}\right)^{2}}{2}-\sin ^{2} F,
$$

satisfies $Q^{\prime}(r)=-r\left(F^{\prime}\right)^{2}$. In particular the function $Q$ is nondecreasing on $(-\infty, 0)$ and nonincreasing on $(0, \infty)$.

By Lemma 2.3, there exist $\beta_{ \pm} \in \mathbb{R}$ such that as $r \rightarrow-\infty$

$$
Q(r)=\beta^{2} r^{-4}+O\left(r^{-6}\right)
$$

Moreover, the case $\beta_{+}=0$ or $\beta_{-}=0$ corresponds to the constant solution (which is trivially monotonic). We will assume that $\beta_{ \pm} \neq 0$, and therefore, $F$ is not the constant solution. Thus, if $|r|$ large, then $Q(r)$ is positive.

We now conclude that $F$ has no critical points. If not, and there exists $r_{0} \in \mathbb{R}$, a critical point for $F$, then $Q\left(r_{0}\right) \leq 0$. In particular, since $Q(r)$ is nondecreasing on $(-\infty, 0)$ from a positive value near $r=-\infty$, we must have that $r_{0}>0$. However, since $F$ is nonconstant, $Q(r)$ is strictly decreasing on $[0, \infty)$ since $Q^{\prime}(r)=-r\left(F^{\prime}\right)^{2}$. Thus, we have $Q(r)<Q\left(r_{0}\right) \leq 0$ for all $r>r_{0}$. This contradicts the fact that $Q(r)>0$ for large positive $r$. Thus, $F$ has no critical points so that $F$ is monotonic on $\mathbb{R}$.

We now prove the existence part of Proposition 2.1.

Lemma 2.6. For each $n \in \mathbb{N}$, there exists a solution $Q_{n}$ to (2.1) that satisfies

$$
\begin{aligned}
Q_{n}(-\infty)=0, \quad Q_{n}(\infty) & =n \pi, \\
\forall r, \quad Q_{n}(r)+Q_{n}(-r) & =n \pi .
\end{aligned}
$$

The proof of Lemma 2.6 now follows from the previous lemmas and a classical shooting argument sketched in [3]. For every $\alpha \in(0, \infty)$, define $F(r, \alpha)$ to be the solution to (2.1) such that

$$
\begin{aligned}
F(0, \alpha) & =\frac{n \pi}{2}, \\
F^{\prime}(0, \alpha) & =\alpha .
\end{aligned}
$$


The variable $\alpha$ is referred to as the shooting variable. We will show that we can choose $\alpha$ so that $F(\infty, \alpha)=n \pi$. Note that if $F(\infty, \alpha)=n \pi$ for some $\alpha$, then the symmetry $F \mapsto n \pi-F$ of the equation yields $F(-r, \alpha)+F(r, \alpha)=n \pi$ so that $F(-\infty, \alpha)=0$. Thus, to prove Lemma 2.6, it suffices to show there exists $\alpha^{*} \in(0, \infty)$ such that

$$
F\left(\infty, \alpha^{*}\right)=n \pi .
$$

We then set $Q_{n}(r)=F\left(r, \alpha^{*}\right)$.

Define

$$
A:=\left\{\alpha \in(0, \infty): \lim _{r \rightarrow \infty} F(r, \alpha)<n \pi\right\}
$$

The proof of Lemma2.6requires a few claims.

Claim 2.7. There exists $\alpha_{0}>0$ so that $\left(0, \alpha_{0}\right) \subset A$.

Proof. For $\alpha \in(0, \infty)$, we denote

$$
Q(r, \alpha)=\left(r^{2}+1\right) \frac{\left(F^{\prime}(r, \alpha)\right)^{2}}{2}-\sin ^{2} F(r, \alpha)
$$

The proof is split into two cases depending on whether $n$ is odd or even.

Case 1. We first consider the case that $n$ is odd. Then we may take $\alpha_{0}=\sqrt{2}$. Indeed, if $\alpha \in(0, \sqrt{2})$, then

$$
Q(0, \alpha)=\frac{\alpha^{2}}{2}-\sin ^{2}\left(\frac{n \pi}{2}\right)=\frac{\alpha^{2}}{2}-1<0
$$

Since $Q(r, \alpha)$ is decreasing on $(0, \infty)$, we must have $Q(r, \alpha)<0$ for all $r>0$. This implies that $F\left(r_{0}, \alpha\right) \neq n \pi$ for all $r_{0} \in(0, \infty)$. The case that $F(r, \alpha) \rightarrow n \pi$ as $r \rightarrow \infty$ is also impossible since then $Q(r, \alpha)>0$ for $r$ sufficiently large (see the proof of Lemma 2.5). Thus, if $n$ is odd, we have $(0, \sqrt{2}) \subset A$.

Case 2. We now consider the case that $n$ is even. In particular, $\frac{n \pi}{2}=l \pi$ for some $l \in \mathbb{N}$. We first note that for every $\alpha \in(0, \infty), F(\cdot, \alpha)$ is increasing until $F$ leaves the strip $\left(l \pi,\left(l+\frac{1}{2}\right) \pi\right)$. Indeed, if $F$ attains a local maximum for some $r_{0}$ with $F\left(r_{0}, \alpha\right) \in\left(l \pi,\left(l+\frac{1}{2}\right) \pi\right)$, then (2.1) implies

$$
F^{\prime \prime}\left(r_{0}, \alpha\right)=\frac{\sin 2 F\left(r_{0}, \alpha\right)}{r_{0}^{2}+1}>0 \text {. }
$$

Thus, $F(\cdot, a)$ is increasing as long as $F \in\left(l \pi,\left(l+\frac{1}{2}\right) \pi\right)$.

Note that since $\frac{n \pi}{2}=l \pi$ for some integer $l$, we have

$$
Q(0, \alpha)=\frac{\alpha^{2}}{2}
$$

We recall that $\left[\left(r^{2}+1\right) Q(r, \alpha)\right]^{\prime}=-2 r \sin ^{2} F(r, \alpha)$ so that

$$
Q(r, \alpha) \leq \frac{Q(0, \alpha)}{r^{2}+1}=\frac{\alpha^{2}}{2\left(r^{2}+1\right)} .
$$

Thus, for all $\alpha$ sufficiently small, we have

$$
F^{\prime}(r, \alpha)^{2}=\frac{2 Q(r, \alpha)}{\left(r^{2}+1\right)^{2}}+\frac{2}{r^{2}+1} \sin ^{2} F(r, \alpha)<\frac{4}{r^{2}} .
$$

Moreover, by continuity of the initial value problem, for $\alpha$ sufficiently small, we can also ensure that

$$
F(r, \alpha)<\left(l+\frac{1}{6}\right) \pi, \quad r \in[0,1] .
$$

Fix $\alpha \in\left(0, \alpha_{0}\right)$ with $\alpha_{0}$ small to be chosen, and suppose that $F(r, \alpha)$ leaves the strip $\left(l \pi,\left(l+\frac{1}{2}\right) \pi\right)$ (if not then $\alpha \in A$ trivially). Since $F(\cdot, \alpha)$ is increasing until it reaches $\left(l+\frac{1}{2}\right) \pi$, there exist $1<r_{1}<r_{2}$ such that

$$
\begin{aligned}
& F\left(r_{1}, \alpha\right)=\left(l+\frac{1}{6}\right) \pi, \\
& F\left(r_{2}, \alpha\right)=\left(l+\frac{1}{4}\right) \pi .
\end{aligned}
$$


Then the fundamental theorem of calculus and (2.12) imply that

$$
\frac{\pi}{4}-\frac{\pi}{6}=\int_{r_{1}}^{r_{2}} F^{\prime}(r, \alpha) d r<2 \log \left(r_{2} / r_{1}\right),
$$

so that

$$
r_{2}-r_{1}>\left(e^{\pi / 24}-1\right) r_{1}>e^{\pi / 24}-1
$$

By (2.11)

$$
\begin{aligned}
\left(r_{2}^{2}+1\right) Q\left(r_{2}, \alpha\right) & =\left(r_{1}^{2}+1\right) Q\left(r_{1}, \alpha\right)-2 \int_{r_{1}}^{r_{2}} r \sin ^{2} F(r, \alpha) d r \\
& \leq Q(0, \alpha)-\frac{1}{2} \int_{r_{1}}^{r_{2}} r d r \\
& =\frac{\alpha^{2}}{2}-\frac{1}{4}\left(r_{2}^{2}-r_{1}^{2}\right) \\
& <\frac{\alpha_{0}^{2}}{2}-\frac{e^{\pi / 24}-1}{8} .
\end{aligned}
$$

Thus, if we choose $\alpha_{0}$ so that $\alpha_{0}^{2}<\frac{e^{\pi / 24}-1}{8}$, we have, for all $\alpha \in\left(0, \alpha_{0}\right), Q\left(r_{2}, \alpha\right)<0$. Since $Q(r, \alpha)$ is decreasing on $(0, \infty)$, it follows that $Q(r, \alpha)<0$ for all $r>r_{2}$. Thus, we cannot have $F(r, \alpha)=(l+1) \pi$ for any $r \in(0, \infty]$ so that

$$
F(\infty, \alpha)<n \pi
$$

Thus, if $\alpha_{0}$ is sufficiently small, $\alpha \in A$ for all $\alpha \in\left(0, \alpha_{0}\right)$.

Claim 2.8. The set $A$ is open.

We recall that

Proof. Let $\alpha_{0} \in A$. We consider two cases.

Case 1. In this case, we assume that there exists $m<n$ such that

$$
F\left(\infty, \alpha_{0}\right)=\left(m+\frac{1}{2}\right) \pi
$$

We first note that for all $r \geq 0$

$$
F\left(r, \alpha_{0}\right)<(m+1) \pi
$$

Indeed, if this were not the case, then, since $F\left(r, \alpha_{0}\right)$ is not constant and $F\left(\infty, \alpha_{0}\right)<(m+1) \pi$, there exist $r_{1}<r_{2}<r_{3}$ such that

$$
\begin{aligned}
F\left(r_{1}, \alpha_{0}\right)=F\left(r_{3}, \alpha_{0}\right) & =(m+1) \pi, \\
F^{\prime}\left(r_{1}, \alpha_{0}\right) \neq 0, \quad F^{\prime}\left(r_{2}, \alpha_{0}\right) & =0, \quad F^{\prime}\left(r_{3}, \alpha_{0}\right) \neq 0 .
\end{aligned}
$$

In particular, $Q\left(r_{2}, \alpha_{0}\right) \leq 0$. But since $Q(r, \alpha)$ is decreasing on $[0, \infty)$, it follows that $Q\left(r_{3}, \alpha_{0}\right)<0$ which is a contradiction to our choice of $r_{3}$. Thus, for all $r \geq 0$

$$
F\left(r, \alpha_{0}\right)<(m+1) \pi
$$

Since $F\left(\infty, \alpha_{0}\right)=\left(m+\frac{1}{2}\right) \pi$ and $F^{\prime}\left(r, \alpha_{0}\right)=o\left(r^{-1}\right)$ (see Lemma 2.2), there exists $R_{0}=R_{0}\left(\alpha_{0}\right)$ large so that

$$
Q\left(R_{0}, \alpha_{0}\right)<0 \text {. }
$$

By continuous dependence of $F(\cdot, \alpha)$ on $\alpha$, we can ensure that for all $\alpha$ is a small neighborhood of $\alpha_{0}$ we have

$$
\begin{aligned}
F(r, \alpha) & <(m+1) \pi, \quad r \in\left[0, R_{0}\right], \\
Q\left(R_{0}, \alpha\right) & <0 .
\end{aligned}
$$

Since $Q(r, \alpha)$ is decreasing on [0, $\infty)$, (2.15) implies for all $\alpha$ sufficiently close to $\alpha_{0}, Q(r, \alpha)<0$ for all $r \geq R_{0}$. In particular, $F(r, \alpha) \neq l \pi$ for any $l \in \mathbb{N}$ and all $r \in\left[R_{0}, \infty\right]$. This along with (2.14) implies that $F(\infty, \alpha)<(m+1) \pi$. Thus, for all $\alpha$ sufficiently close to $\alpha_{0}$, we have $\alpha \in A$ as desired. 
Case 2. In this case we assume that there exists $m<n$ such that

$$
F\left(\infty, \alpha_{0}\right)=m \pi \text {. }
$$

We first note that in this case, we have $Q\left(r, \alpha_{0}\right)=O\left(r^{-4}\right)$ (see the proof of Lemma 2.5), so that,

$$
\lim _{r \rightarrow \infty}\left(r^{2}+1\right) Q\left(r, \alpha_{0}\right)=0 \text {. }
$$

Let $\epsilon_{0}>0$ to be chosen later. Then by (2.16), there exists $R_{0}=R_{0}\left(\epsilon_{0}\right)>1$ such that

$$
\left(r^{2}+1\right) Q\left(r, \alpha_{0}\right)<\epsilon_{0}, \quad r \geq R_{0} .
$$

For $\alpha$ in a small (depending on $\epsilon_{0}$ ) neighborhood of $\alpha_{0}$, we have

$$
\begin{aligned}
Q(0, \alpha) & <2 Q\left(0, \alpha_{0}\right) \\
\left(R_{0}^{2}+1\right) Q\left(R_{0}, \alpha\right) & <2 \epsilon_{0}, \\
\frac{n \pi}{2} \leq F(r, \alpha) & <m \pi, \quad r \in\left[0, R_{0}\right] .
\end{aligned}
$$

We now claim that for each such $\alpha$, we have

$$
F(\infty, \alpha) \leq\left(m+\frac{1}{2}\right) \pi
$$

Let $\alpha$ be sufficiently close to $\alpha_{0}$ so that (2.17), (2.18), and (2.19) are satisfied, and assume that

$$
F(\infty, \alpha)>m \pi \text {. }
$$

Then by (2.19), there exists $r_{0} \geq R_{0}$ such that $F\left(r_{0}, \alpha\right)=m \pi$. Since $F(\cdot, \alpha)$ is increasing as long as $F(\cdot, \alpha)$ is in the strip $\left(m \pi,\left(m+\frac{1}{2}\right) \pi\right)$ (see the proof of Claim 2.7), there exist $r_{1}, r_{2}>R_{0}$ such that $r_{1}<r_{2}$ and

$$
\begin{aligned}
& F\left(r_{1}, \alpha\right)=\left(m+\frac{1}{6}\right) \pi, \\
& F\left(r_{2}, \alpha\right)=\left(m+\frac{1}{4}\right) \pi .
\end{aligned}
$$

As in the proof of Claim 2.7, by (2.17) we have

$$
F^{\prime}(r, \alpha)^{2} \leq \frac{2 Q(0, \alpha)}{\left(r^{2}+1\right)^{2}}+\frac{2}{r^{2}+1} \sin ^{2} F(r, \alpha) \leq \frac{C^{2}\left(\alpha_{0}\right)}{r^{2}},
$$

for some positive constant $C\left(\alpha_{0}\right)$. By our choice of $r_{1}, r_{2},(2.21)$, and the fundamental theorem of calculus, we deduce that

$$
\frac{\pi}{4}-\frac{\pi}{6}=\int_{r_{1}}^{r_{2}} F^{\prime}(r, \alpha) d r \geq C\left(\alpha_{0}\right) \log \left(r_{2} / r_{1}\right),
$$

whence for some (possibly small) constant $c\left(\alpha_{0}\right)>0$

$$
r_{2}-r_{1} \geq c\left(\alpha_{0}\right) \text {. }
$$

By the relation $\left[\left(r^{2}+1\right) Q(r, \alpha)\right]^{\prime}=-2 r \sin ^{2} F(r, \alpha)$ and $(2.18)$, we have

$$
\begin{aligned}
\left(r_{2}^{2}+1\right) Q\left(r_{2}, \alpha\right) & =\left(r_{1}^{2}+1\right) Q\left(r_{1}, \alpha\right)-2 \int_{r_{1}}^{r_{2}} r \sin ^{2} F(r, \alpha) d r \\
& <2 \epsilon_{0}-\frac{1}{2} \int_{r_{1}}^{r_{2}} r d r \\
& <2 \epsilon_{0}-\frac{1}{2}\left(r_{2}^{2}-r_{1}^{2}\right) \\
& <2 \epsilon_{0}-\frac{1}{2} c\left(\alpha_{0}\right) .
\end{aligned}
$$

By initially choosing $\epsilon_{0}$ sufficiently small (depending only on $\alpha_{0}$ ), we see that if $\alpha$ is sufficiently close to $\alpha_{0}$ so that (2.17), (2.18), and (2.19) are satisfied, we have $Q\left(r_{2}, \alpha\right)<0$. Thus, $Q(r, \alpha)<0$ for all $r \geq r_{2}$. Hence, for 
any $l>m, F(r, \alpha) \neq l \pi$ for all $r \in\left[R_{0}, \infty\right]$. This along with (2.19) proves that $F(r, \alpha) \leq\left(m+\frac{1}{2}\right) \pi$ for all $r \geq 0$ which establishes (2.20). Thus, all $\alpha$ sufficiently close to $\alpha_{0}$ are in $A$ which finishes the proof of Claim 2.8 .

Claim 2.9. There exists $\alpha_{1}>0$ such that $\left(\alpha_{1}, \infty\right) \subseteq A^{c}$.

Proof. We first note that if $\alpha>0$ and if $F(r, \alpha)=n \pi$ for some $r>0$, then $F(\infty, \alpha)>n \pi$. Indeed, suppose $F\left(r_{0}, \alpha\right)=n \pi$ for some $r_{0}>0$ and $F(\infty, \alpha) \leq n \pi$. Since $F(r, \alpha)$ is not the constant function, there exist $r_{0}<r_{1}<r_{2} \leq \infty$ such that $F^{\prime}\left(r_{1}, \alpha\right)=0$ and $F\left(r_{2}, \alpha\right)=n \pi$. We then have that $Q\left(r_{1}, \alpha\right) \leq 0$ and $Q\left(r_{2}, \alpha\right)>0$. This contradicts the fact that $Q(r, \alpha)$ is decreasing on [0, $\infty)$. Thus, if $F(r, \alpha)=n \pi$ for some $r>0$, then $F(\infty, \alpha)>n \pi$. In particular, we have shown that

$$
\left\{a>0: F\left(r_{0}, a\right)=n \pi \text { for some } r_{0}>0\right\} \subset A^{c} .
$$

Thus, the proof of Claim 2.9 is reduced to showing that there exists $\alpha_{1}>0$ such that

$$
\left(\alpha_{1}, \infty\right) \subseteq\left\{a>0: F\left(r_{0}, a\right)=n \pi \text { for some } r_{0}>0\right\} .
$$

The idea of the proof is now simple. If the initial velocity $\alpha$ is large enough, then $F(r, \alpha)=n \pi$ for some $r>0$ so that $\alpha \in A^{c}$. To make this argument precise, we need the precise asymptotics of $F(r, \alpha)$ for $r$ near $r=0$. First we change variables and set $x=\operatorname{arcsinh} r$. Then $F(x, \alpha):=F(r(x), \alpha)$ satisfies $F(0, \alpha)=n \pi / 2$, $F^{\prime}(0, \alpha)=\alpha$ and

$$
F^{\prime \prime}+\tanh x F^{\prime}-\sin 2 F=0 .
$$

We first claim there exists $x_{0}>0$ small such that for all $\alpha>0$

$$
\|F(\cdot, \alpha)\|_{C^{1}\left(\left[0, x_{0}\right]\right)} \leq n \pi+4 \alpha .
$$

Indeed, we solve (2.22) near $x=0$ by a contraction mapping argument. Let $X=C^{1}\left(\left[0, x_{0}\right]\right)$ where $x_{0}$ is to be chosen later. Define $\Phi: X \rightarrow X$ by

$$
\Phi F(x)=\frac{n \pi}{2}+\alpha x+\int_{0}^{x}(x-y)\left[\sin 2 F(y)-\tanh y F^{\prime}(y)\right] d y
$$

If $x_{0}$ is chosen so small so that tanh $y \leq 2 y$ for $y \in\left[0, x_{0}\right]$, then it is easy to verify that for all $F, G \in X$ and for some absolute constant $C>0$

$$
\begin{aligned}
\|\Phi F\|_{X} & \leq \frac{n \pi}{2}+2 \alpha+C x_{0}\|F\|_{X}, \\
\|\Phi F-\Phi G\|_{X} & \leq C x_{0}\|F-G\|_{X} .
\end{aligned}
$$

Now fix $x_{0}$ smaller if necessary so that $x_{0}<1 /(8 C)$. Then, we may contract in the ball $B_{X}(0, n \pi+4 \alpha)$ and find a unique fixed point (namely $F(x, \alpha)$ ) of $\Phi$. This shows that there exists $x_{0}$ small and independent of $\alpha$ such that $\|F(\cdot, \alpha)\|_{C^{1}\left(\left[0, x_{0}\right]\right)} \leq n \pi+4 \alpha$ as desired.

We now conclude that if $\alpha$ is sufficiently large (depending on $x_{0}$ ), then in fact $F\left(x_{0}, \alpha\right) \geq n \pi$ where $x_{0}$ was defined previously. We write for $x \in\left[0, x_{0}\right]$

$$
F(x, \alpha)=\frac{n \pi}{2}+\alpha x+\int_{0}^{x}(x-y)\left[\sin 2 F(y, \alpha)-\tanh y F^{\prime}(y, \alpha)\right] d y .
$$

Then by (2.23), for some constant $C>0$ and by choosing $x_{0}$ smaller if necessary, we have

$$
\begin{aligned}
\left|F\left(x_{0}, \alpha\right)\right| & \geq \frac{n \pi}{2}+\alpha x_{0}-C x_{0}^{2}\|F(\cdot, \alpha)\|_{C^{1}\left(\left[0, x_{0}\right]\right)} \\
& \geq \frac{n \pi}{2}\left(1-C x_{0}^{2}\right)+\alpha x_{0}\left(1-4 x_{0} C\right) \\
& \geq \frac{n \pi}{4}+\frac{\alpha x_{0}}{2} .
\end{aligned}
$$

This shows that for all $\alpha \geq 2 n \pi / x_{0}, F\left(x_{0}, \alpha\right) \geq n \pi$, i.e. $\alpha \in\left\{a>0: F\left(r_{0}, a\right)=n \pi\right.$ for some $\left.r_{0}>0\right\} \subset A^{c}$.

Proof of Lemma 2.6 By Claim 1 and Claim 3,

$$
\alpha^{*}:=\sup A \in(0, \infty)
$$


By Claim 2, $\alpha^{*} \notin A$. Suppose that $\alpha^{*} \in\{\alpha \in(0, \infty): F(\infty, \alpha)>n \pi\}$. Then by continuous dependence of initial data, all $\alpha$ near $\alpha^{*}$ are also in $\{\alpha \in(0, \infty): F(\infty, \alpha)>n \pi\}$. This, however, contradicts the facts that $\alpha^{*}=\sup A$ and that $A$ is open (by Claim 2). Thus, $F\left(\infty, \alpha^{*}\right)=n \pi$, and we are done.

2.2. Uniqueness of the Harmonic Map. In this section we show uniqueness of the harmonic map constructed in the previous section which concludes the proof of Proposition 2.1.

Lemma 2.10. Let $F_{1}$ and $F_{2}$ solve (2.1) and assume that for $j=1,2$

$$
F_{j}(-\infty)=0, \quad F_{j}(\infty)=n \pi .
$$

Then $F_{1}=F_{2}$.

Proof. Since any $F$ that solves (2.1) and connects 0 to $n \pi$ must be increasing, we may make a change of variables and consider $F$ as the dependent variable and $p=\frac{d F}{d x}$ as the dependent variable, where $x=\operatorname{arcsinh} r$. Thus, the equation solved by $p$ is

$$
p \frac{d p}{d F}+(\tanh x) p-\sin 2 F=0 .
$$

Suppose towards a contradiction, that we have two different solutions $F_{1}, F_{2}$. These determine two $C^{\infty}$ diffeomorphisms $x_{1}, x_{2}:(0, n \pi) \rightarrow(-\infty, \infty)$ by the condition $F_{j} \circ x_{j}$ is the identity on $(0, n \pi)$. Then we have

$$
p_{j}(F) \frac{d p_{j}}{d F}+\left(\tanh x_{j}(F)\right) p_{j}(F)-\sin 2 F=0, \quad j=1,2 .
$$

Set $\phi(F)=p_{2}(F)-p_{1}(F)$. Subtracting the equation satisfied by $p_{1}$ from the equation satisfied by $p_{2}$ and rearranging, we have

$$
\begin{aligned}
0 & =p_{2} \frac{d p_{2}}{d F}-p_{1} \frac{d p_{1}}{d F}+\tanh x_{2} p_{2}-\tanh x_{1} p_{1} \\
& =p_{2} \frac{d \phi}{d F}+\left(\frac{d p_{1}}{d F}+\tanh x_{2}\right) \phi-\left(\tanh x_{1}-\tanh x_{2}\right) p_{1} .
\end{aligned}
$$

Define $q=p_{2}^{-1}\left(\frac{d p_{1}}{d F}+\tanh x_{2}\right), f=\left(\tanh x_{2}-\tanh x_{1}\right) p_{1} p_{2}^{-1}$. Then $\phi$ satisfies

$$
\phi^{\prime}+q \phi=-f \Longrightarrow\left(-\phi e^{-Q}\right)^{\prime}=f,
$$

where $Q(F)=\int_{F}^{F_{0}} q(\bar{F}) d \bar{F}$ for any choice of $F_{0} \in(0, n \pi)$. Hence, we have that

$$
\phi(F)=e^{Q(F)} \phi\left(F_{0}\right)+\int_{F}^{F_{0}} e^{Q(F)-Q(\bar{F})} f(\bar{F}) d \bar{F} .
$$

We now make an observation based on (2.26). Note that if $p_{2}\left(F_{0}\right)>p_{1}\left(F_{0}\right)$ and $x_{2}\left(F_{0}\right)>x_{2}(F)$ imply that $p_{2}(F)>p_{1}(F)$ and $x_{2}(F)>x_{1}(F)$ for all $F \leq F_{0}$. Indeed, suppose $F_{1}<F_{0}$ and $p_{2}(F) \geq p_{1}(F)$ for all $F_{1} \leq F \leq F_{0}$. Then from the definition of $p_{j}$, we have for all $F_{1} \leq F \leq F_{0}$

$$
p_{2}(F) \geq p_{1}(F) \Longrightarrow\left(x_{2}(F)-x_{1}(F)\right)^{\prime} \leq 0 .
$$

This implies upon integrating that

$$
0<x_{2}\left(F_{0}\right)-x_{1}\left(F_{0}\right) \leq x_{2}(F)-x_{1}(F), \quad F_{1} \leq F \leq F_{0} .
$$

Since $\tanh x$ is increasing on $(-\infty, \infty)$,

$$
x_{2}(F)>x_{1}(F), \quad F_{1} \leq F \leq F_{0} \Longrightarrow f(F)>0, \quad F_{1} \leq F \leq F_{0} .
$$

Hence by (2.26)

$$
p_{2}(F)>p_{1}(F), \quad F_{1} \leq F \leq F_{0} .
$$

Thus, if $p_{2}(F) \geq p_{1}(F)$ for all $F_{1} \leq F \leq F_{0}$, we must in fact have the strict inequalities $p_{2}(F)>p_{1}(F)$ and $x_{2}(F)>x_{1}(F)$ for $F_{1} \leq F \leq F_{0}$. By continuity, we see that $p_{2}(F)>p_{1}(F)$ and $x_{2}(F)>x_{1}(F)$ for all $F \leq F_{0}$. 
By Lemma 2.3, a solution $F$ to (2.1) such that $F(-\infty)=0, F(\infty)=n \pi$ satisfies for unique $a, b>0$,

$$
\begin{gathered}
F(x)-n \pi \sim-a e^{-2 x}+a \frac{2}{5} e^{-4 x}, \quad \text { as } x \rightarrow \infty, \\
F(x) \sim b e^{2 x}-b \frac{2}{5} e^{4 x}, \quad \text { as } x \rightarrow-\infty .
\end{gathered}
$$

It follows that $p$ satisfies

$$
\begin{aligned}
p & \sim 2 a e^{-2 x}-a \frac{8}{5} e^{-4 x} \\
& \sim 2(n \pi-F)-a \frac{4}{5} e^{-4 x} \\
& \sim 2(n \pi-F)-a^{-1} \frac{4}{5}(n \pi-F)^{2},
\end{aligned}
$$

as $F \rightarrow n \pi^{-}$. Similarly, we have

$$
p \sim 2 F-b^{-1} \frac{4}{5} F^{2}
$$

as $F \rightarrow 0^{+}$. Suppose $F_{2}$ has coefficients $a_{2}, b_{2}>0$ and $F_{1}$ has coefficients $a_{1}, b_{1}>0$ where (without loss of generality) $a_{2}>a_{1}$. Then clearly $x_{2}(F)>x_{1}(F)$ for all $F$ sufficiently close to $n \pi$ since for $x$ large

$$
F_{2}(x) \sim n \pi-a_{2} e^{-2 x}<n \pi-a_{1} e^{-2 x} \sim F_{1}(x) .
$$

Moreover, we have $p_{2}(F)>p_{1}(F)$ for $F$ sufficiently close to $n \pi$ by our previous calculation

$$
\begin{aligned}
p_{2}(F) & \sim 2(n \pi-F)-a_{2}^{-1} \frac{4}{5}(n \pi-F)^{2} \\
& >2(n \pi-F)-a_{1}^{-1} \frac{4}{5}(n \pi-F)^{2} \sim p_{1}(F) .
\end{aligned}
$$

Thus, by our observation following (2.26), we have $p_{2}(F)>p_{1}(F)$ and $x_{2}(F)>x_{1}(F)$ for all $F \in(0, n \pi)$. In particular, the constraint $x_{2}(F)>x_{1}(F)$ for all $F \in(0, n \pi)$ implies that $b_{1}>b_{2}$. But then for $F$ near 0

$$
\begin{aligned}
p_{1}(F) & \sim 2 F-b_{1}^{-1} \frac{4}{5} F^{2} \\
& >2 F-b_{2}^{-1} \frac{4}{5} F^{2} \sim p_{2}(F),
\end{aligned}
$$

which contradicts $p_{2}(F)>p_{1}(F)$ for all $F \in(0, n \pi)$. Thus, no two distinct solutions $F_{1}, F_{2}$ exist. This completes the proof.

\section{Strichartz Estimates for the Free Wave Equation on Wormholes}

In this section we establish Strichartz estimates for radial solutions to the free wave equation on the $(d+1)$-dimensional wormhole $\mathcal{M}^{d+1}=\left\{(r, \omega): r \in \mathbb{R}, \omega \in \mathbb{S}^{d}\right\}$ with metric $g$ satisfying

$$
d s^{2}=d r^{2}+\left(r^{2}+1\right) d \Omega_{\mathbb{S}^{d}}^{2}(\omega) .
$$

Here $d \Omega_{\mathbb{S}^{d}}^{2}$ is the line element on $\mathbb{S}^{d}$ corresponding to the usual round metric. When we say radial functions we mean functions $f: \mathcal{M}^{d+1} \rightarrow \mathbb{R}$ with $f=f(r)$. These Strichartz estimates will be used in Section 4 and Section 5 to establish a small data theory for (1.6). However, the results and methods of this section are independent of all other sections in this work and may be of interest in their own right.

For the remainder of the section, we fix $d \geq 2$ and drop the superscript by writing $\mathcal{M}$ instead of $\mathcal{M}^{d+1}$. We denote $\mathcal{H}\left(\mathbb{R} ;\left(r^{2}+1\right)^{d / 2} d r\right)$ simply by $\mathcal{H}$. For an interval $I$, we denote the spatial norms on $\mathcal{M}$ and spacetime 
norms on $I \times \mathcal{M}$ by

$$
\begin{aligned}
\|f\|_{L^{p}} & :=\left(\int|f(r)|^{p}\left(r^{2}+1\right)^{d / 2} d r\right)^{1 / p}, \\
\|u\|_{L_{t}^{p} L_{x}^{q}(I)} & :=\left(\int_{\mathbb{R}}\left(\int_{\mathbb{R}}|u(t, r)|^{q}\left(r^{2}+1\right)^{d / 2} d r\right)^{p / q} d t\right)^{1 / p} .
\end{aligned}
$$

Since we only consider radial functions on $\mathcal{M}$, we abuse notation slightly and let $\Delta_{g}$ denote the radial part of the Laplace operator on $\mathcal{M}$,

$$
\Delta_{g} f(r)=\partial_{r}^{2} f+\frac{d r}{r^{2}+1} \partial_{r} f
$$

Let $I$ be an interval with $0 \in I$. Let $F: I \times \mathbb{R} \rightarrow \mathbb{R}$, and let $u=u(t, r)$ solve the inhomogeneous wave equation

$$
\begin{aligned}
& \partial_{t}^{2} u-\Delta_{g} u=F, \quad(t, r) \in I \times \mathbb{R} . \\
& \vec{u}(0)=\left(u_{0}, u_{1}\right) \in \mathcal{H} .
\end{aligned}
$$

We say that a triple $(p, q, \gamma)$ is admissible if

$$
p>2, q \geq 2, \quad \frac{1}{p}+\frac{d+1}{q}=\frac{d+1}{2}-\gamma, \quad \frac{1}{p} \leq \frac{d}{2}\left(\frac{1}{2}-\frac{1}{q}\right) .
$$

The main result of this section is the following family of Strichartz estimates for (3.1).

Proposition 3.1. Let $(p, q, \gamma)$ and $(a, b, \rho)$ be admissible triples. Then any solution $u$ to (3.1) satisfies

$$
\left\|\left|\left\|\left.\nabla\right|^{1-\gamma} u\right\|_{L_{t}^{p} L_{x}^{q}(I)}+\left\||\nabla|^{-\gamma} \partial_{t} u\right\|_{L_{t}^{p} L_{x}^{q}(I)} \lesssim\|\vec{u}(0)\|_{\mathcal{H}}+\left\|\left|\left\|\left.\nabla\right|^{\rho} F\right\|_{L_{t}^{a^{\prime}} L_{x}^{b^{\prime}(I)}} .\right.\right.\right.\right.
$$

It is well known (see for example [11] [26] [27]) that by a standard argument using Littlewood-Paley theory (for our wormhole geometry see [28]) and $T T^{*}$ arguments, establishing Proposition 3.1 can be reduced to proving the following frequency localized dispersive estimate: let $E$ denote the spectral measure for $-\Delta_{g}$ (restricted to radial functions). For a standard Littlewood-Paley cutoff $\varphi \in C_{0}^{\infty}(\mathbb{R})$ with support in $(1 / 2,2)$, define (via the functional calculus)

$$
\varphi\left(2^{-j} \sqrt{-\Delta_{g}}\right)=\int_{0}^{\infty} \varphi\left(2^{-j} \sqrt{\lambda}\right) E(d \lambda)
$$

Then for all $f \in C_{0}^{\infty}(\mathbb{R})$,

$$
\left\|e^{ \pm i t} \sqrt{-\Delta_{g}} \varphi\left(2^{-j} \sqrt{-\Delta_{g}}\right) f\right\|_{L^{\infty}} \lesssim 2^{\frac{d+2}{2}}\left(2^{-j}+|t|\right)^{-\frac{d}{2}}\|f\|_{L^{1}} .
$$

The proof of (3.2) draws heavily from the works [23] [24]. In these works, the authors prove dispersive estimates for free waves on a manifold with metric of the form

$$
d s^{2}=d r^{2}+R^{2}(r) d s_{\Omega}^{2}(\omega), \quad r \in \mathbb{R},
$$

where $d s_{\Omega}^{2}(\omega)$ is the metric on a compact embedded Riemannian manifold $\Omega \subset \mathbb{R}^{N}$ with dimension $d \geq 1$. The function $R(r)$ is assumed to be asymptotically conic:

$$
R(r)=|r|\left(1+O\left(r^{-1}\right)\right), \quad \text { as } r \rightarrow \pm \infty .
$$

Note that in the case of the wormhole geometry, $\Omega=\mathbb{S}^{d}$ and $R(r)=\langle r\rangle$. In particular, the authors proved weighted $L^{1} \rightarrow L^{\infty}$ type estimates for data of the form $f(r) Y_{n}(\omega)$ where $Y_{n}$ are eigenfunctions of $-\Delta_{\Omega}$. For the $n=0$ case (i.e. a radial solution), they established the dispersive estimate

$$
\left\|e^{ \pm i t} \sqrt{-\Delta_{g}} f(r)\right\|_{L^{\infty}} \lesssim|t|^{-d / 2}\left(\|f\|_{L^{1}}+\left\|f^{\prime}\right\|_{L^{1}}\right) \text {. }
$$

In our proof of 3.2, we refine their methods for the case of frequency localized data.

In what follows, we use the standard Japanese bracket notation $\langle r\rangle=\left(r^{2}+1\right)^{1 / 2}$. One readily verifies that

$$
-\Delta_{g} f(r)=\left(\langle r\rangle^{-d / 2} H\langle r\rangle^{d / 2}\right) f(r)
$$


where $H$ is the Schrodinger operator on $\mathbb{R}$ given by

$$
H=-\frac{d^{2}}{d r^{2}}+V, \quad V(r)=\frac{d(d-4)}{4} r^{2}\langle r\rangle^{-4}+\frac{d}{2}\langle r\rangle^{-2} .
$$

Note that the potential $V$ satisfies

$$
V(r)=\frac{d(d-2)}{2 r^{2}}+O\left(r^{-3}\right)
$$

as $r \rightarrow \pm \infty$ with natural derivative bounds. We denote the following resolvents $R(z)=\left(-\Delta_{g}-z\right)^{-1}$ and $R_{H}(z)=(H-z)^{-1}$ for $z \notin \sigma\left(-\Delta_{g}\right)=\sigma(H)=[0, \infty)$. We note that the decay of $V$ implies that the spectrum of $H$ in $(0, \infty)$ is purely absolutely continuous (in fact, absolute continuity follows from the following explicit formula for the spectral measure).

Via Stone's theorem, we can write (as an identity of Schwartz kernels)

$$
\begin{aligned}
E\left(d \lambda^{2}\right)(r, \rho) & =\frac{\lambda}{\pi i} \lim _{\epsilon \rightarrow 0^{+}}\left(R\left(\lambda^{2}+i \epsilon\right)-R\left(\lambda^{2}-i \epsilon\right)\right)(r, \rho) d \lambda \\
& =\frac{\lambda}{\pi i} \lim _{\epsilon \rightarrow 0^{+}}\langle r\rangle^{-d / 2}\left(R_{H}\left(\lambda^{2}+i \epsilon\right)-R_{H}\left(\lambda^{2}-i \epsilon\right)\right)(r, \rho)\langle\rho\rangle^{d / 2} d \lambda .
\end{aligned}
$$

The final limit may be evaluated 'explicitly' by using the fact that

$$
\begin{aligned}
\lim _{\epsilon \rightarrow 0^{+}} \frac{1}{2 \pi i}\left(R_{H}\left(\lambda^{2}+i \epsilon\right)-R_{H}\left(\lambda^{2}-i \epsilon\right)\right)(r, \rho)= & \mathfrak{J}\left[\frac{f_{+}(r, \lambda) f_{-}(\rho, \lambda)}{W(\lambda)}\right] \chi_{[r>\rho]} \\
& +\mathfrak{J}\left[\frac{f_{-}(r, \lambda) f_{+}(\rho, \lambda)}{W(\lambda)}\right] \chi_{[r<\rho]},
\end{aligned}
$$

where $f_{ \pm}(\cdot, \lambda)$ are the Jost solutions which satisfy

$$
\begin{array}{r}
H f_{ \pm}(r, \lambda)=\lambda^{2} f_{ \pm}(r, \lambda), \\
f_{ \pm}(r, \lambda) \sim e^{ \pm i r \lambda} \quad \text { as } r \rightarrow \pm \infty,
\end{array}
$$

and

$$
W(\lambda)=W\left(f_{-}(\cdot, \lambda), f_{+}(\cdot, \lambda)\right)=f_{+}^{\prime}(\cdot, \lambda) f_{-}(\cdot, \lambda)-f_{+}(\cdot, \lambda) f_{-}^{\prime}(\cdot, \lambda),
$$

is their Wronskian. It is easy to see via a standard contraction argument that $f_{ \pm}(\cdot, \lambda)$ exist provided $V \in L^{1}(\mathbb{R})$. In summary, we see that the spectral measure for $-\Delta_{g}$ satisfies

$$
E\left(d \lambda^{2}\right)(r, \rho)=2 \lambda\langle r\rangle^{-d / 2}\left\{\mathfrak{J}\left[\frac{f_{+}(r, \lambda) f_{-}(\rho, \lambda)}{W_{v}(\lambda)}\right] \chi_{[r>\rho]}+\mathfrak{J}\left[\frac{f_{-}(r, \lambda) f_{+}(\rho, \lambda)}{W_{v}(\lambda)}\right] \chi_{[r<\rho]}\right\}\langle\rho\rangle^{d / 2} d \lambda .
$$

Therefore, the estimate (3.2) (and thus, Proposition 3.1) reduces to proving the following oscillatory integral estimate uniformly in $r>\rho$ (the case $r<\rho$ is analagous) which we state as a proposition.

Proposition 3.2. For all $\rho<r$ and $t \in \mathbb{R}$ we have the estimate

$$
\left|\int_{0}^{\infty} e^{ \pm i t \lambda} \varphi\left(2^{-j} \lambda\right) \lambda \mathfrak{J}\left[\frac{f_{+}(r, \lambda) f_{-}(\rho, \lambda)}{W(\lambda)}\right] d \lambda\right| \lesssim(\langle r\rangle\langle\rho\rangle)^{d / 2} 2^{j(d+2) / 2}\left(2^{-j}+|t|\right)^{-d / 2} .
$$

The implied constant depends only on $\varphi$ and $d$.

Note that we absorbed the volume form $\left(r^{2}+1\right)^{d / 2} d r$ implicit in the right hand side of (3.2) into the left hand side in order to conclude that proving the estimate 3.2 reduces to proving Proposition 3.2. To prove Proposition 3.2, we will need asymptotics for $f_{ \pm}(\cdot, \lambda)$ and $W(\lambda)$ for $\lambda$ small. The asymptotics that we require are contained in the following subsection. 
3.1. Scattering Theory for Schrodinger Operators. In this section, we briefly summarize the scattering theory developed in Section 3 of [24] for the Schrödinger operator $H=-\frac{d^{2}}{d r^{2}}+V$ on $\mathbb{R}$ where $V \in C^{\infty}(\mathbb{R})$ is real-valued and such that

$$
V(r)=\frac{d(d-2)}{4} r^{-2}+U(r), \quad U \in C^{\infty}(\mathbb{R} \backslash\{0\}),
$$

with

$$
\left|U^{(k)}(r)\right| \leq C_{k}|r|^{-3-k}, \quad|r| \geq 1
$$

In particular, we summarize the asymptotics for $f_{ \pm}(\cdot, \lambda)$ and $W(\lambda)$ as $\lambda \rightarrow 0$ under a condition on the point spectrum of $H$. This condition will be elaborated on below. In what follows, we assume, as before, that $d \geq 2$.

First, solutions to the zero energy equation with slow decay at $\pm \infty$ were constructed.

Lemma 3.3 (Lemma 3.2 [24]). For $j=0,1$, there exist real-valued solutions $u_{j}^{ \pm}(\cdot)$ to the zero energy equation

$$
-u_{j}^{ \pm}(r)^{\prime \prime}+V(r) u_{j}^{ \pm}(r)=0, \quad r \in \mathbb{R},
$$

such that $W\left(u_{0}^{ \pm}(\cdot), u_{1}^{ \pm}(\cdot)\right)=$ constant, and $u_{j}^{ \pm}$have the asymptotics

$$
\begin{aligned}
& u_{0}^{ \pm}(r)=|r|^{d / 2}\left(1+O\left(|r|^{-1}\right)\right), \quad \text { as } r \rightarrow \pm \infty, \\
& u_{1}^{ \pm}(r)=|r|^{-(d-2) / 2}\left(1+O\left(|r|^{-1}\right)\right), \quad \text { as } r \rightarrow \pm \infty .
\end{aligned}
$$

The $O(\cdot)$ terms behave like symbols under differentiation in $r$.

Definition 3.4. We say that the Schrödinger operator $H$ has 0 as a resonance if

$$
W\left(u_{1}^{+}(\cdot), u_{1}^{-}(\cdot)\right)=0
$$

where $u_{1}^{ \pm}(\cdot)$ are the solutions constructed in Lemma 3.3 This condition is equivalent to the existence of a nonzero solution $f$ to $-f^{\prime \prime}+V f=0$ such that $f$ is asymptotic to $|r|^{-(d-2) / 2}$ at $\pm \infty$.

The previously mentioned condition on the point spectrum of $H$ is that 0 is not a resonance. Next, perturbing in small $\lambda$, for $j=0,1$ a basis of real-valued solutions $u_{j}^{ \pm}(\cdot, \lambda)$ to

$$
-u_{j}^{ \pm}(r, \lambda)^{\prime \prime}+V(r) u_{j}^{ \pm}(r, \lambda)=\lambda^{2} u_{j}^{ \pm}(r, \lambda), \quad r \in \mathbb{R},
$$

was constructed which are well approximated by $u_{j}^{ \pm}$when $|r \lambda| \ll 1$.

Lemma 3.5 (Corollary $3.5[24]$ ). Let $u_{j}^{+}(\cdot)$ be as in Lemma 3.3 There exist solutions $u_{j}^{+}(\cdot, \lambda)$ of $H f=\lambda^{2} f$ with

$$
W\left(u_{1}^{+}(\cdot, \lambda), u_{0}^{+}(\cdot, \lambda)\right)=1,
$$

such that for $j=0,1$ and $r_{0} \leq r \ll \lambda^{-1}$, we have

$$
u_{j}^{+}(r, \lambda)=u_{j}^{+}(r)\left(1+a_{j}^{+}(r, \lambda)\right)
$$

The functions $a_{j}^{+}(\cdot, \lambda)$ satisfy the bounds

$$
\left|\partial_{r}^{l} \partial_{\lambda}^{k} a_{j}^{+}(r, \lambda)\right| \lesssim_{k, l} \begin{cases}\lambda^{2-k}\langle r\rangle^{2-l} \log |\lambda r| & \text { if } d=2, \\ \lambda^{2-k}\langle r\rangle^{2-l} & \text { if } d>2 .\end{cases}
$$

A similar statement holds with $u_{0}^{+}(\cdot, \lambda)$ replaced by $u_{0}^{-}(\cdot, \lambda)$ for $r \leq 0$.

In what follows, $\beta_{d}=\sqrt{\frac{\pi}{2}} e^{i d \pi / 4}$. The outgoing Jost solution for

$$
H_{0}=-\frac{d^{2}}{d r^{2}}+\frac{d(d-2)}{2 r^{2}}
$$

is known explicitly. In particular, we have that the solution to $H_{0} f_{0}(\cdot, \lambda)=\lambda^{2} f_{0}(\cdot, \lambda)$ with $f_{0}(r, \lambda) \sim e^{i \lambda r}$ as $r \rightarrow \infty$ is given by

$$
f_{0}(r, \lambda)=\beta_{d} \sqrt{r \lambda} H_{(d-1) / 2}^{+}(r \lambda)
$$


where $H_{(d-1) / 2}^{+}(z)=J_{(d-1) / 2}(z)+i Y_{(d-1) / 2}(z)$ is the Hankel function. Perturbing off of this explicit solution, we obtain the following asymptotic form for the Jost function $f_{+}(\cdot, \lambda)$. Similar asymptotics hold for $f_{-}(\cdot, \lambda)$.

Lemma 3.6 (Corollary 3.10 [24]). For $\lambda \neq 0, \lambda \ll 1$, and in the range $1 \ll r \ll \lambda^{-1}$, we have

$$
\begin{aligned}
f_{+}(r, \lambda) & =\beta_{d} \sqrt{\lambda r}\left[J_{(d-1) / 2}(r \lambda)(1+O(\lambda))\left(1+O\left(r^{-1}\right)\right)+Y_{(d-1) / 2}(r \lambda) O(\lambda)\left(1+O\left(r^{-1}\right)\right)\right] \\
& +i \beta_{d} \sqrt{\lambda r}\left[Y_{(d-1) / 2}(r \lambda)(1+O(\lambda))\left(1+O\left(r^{-1}\right)\right)+J_{(d-1) / 2}(r \lambda) O(\lambda)\left(1+O\left(r^{-1}\right)\right)\right] .
\end{aligned}
$$

In the range $r \lambda \gtrsim 1$, we have

$$
f_{+}(r, \lambda)=e^{i r \lambda} m_{+}(r, \lambda)
$$

where

$$
m_{+}(r, \lambda)=1+O_{\mathbb{C}}\left(r^{-1} \lambda^{-1}\right)
$$

The $O(\cdot)$ terms are real-valued, the $O_{\mathbb{C}}(\cdot)$ term is complex-valued, and all terms obey the natural bounds with respect to differentiation in $\lambda$ and $r$.

Using the previous lemmas, the following expansions were obtained.

Lemma 3.7 (Corollary 3.6 and Proposition 3.12 [24]). We have the expansions

$$
f_{ \pm}(r, \lambda)=a_{ \pm}(\lambda) u_{0}^{ \pm}(r, \lambda)+b_{ \pm}(\lambda) u_{1}^{ \pm}(r, \lambda),
$$

where the coefficients satisfy with some small $\epsilon>0$ depending on $d$ and with some real constants $\alpha_{0}^{ \pm}, \beta_{0}^{ \pm}$,

$$
\begin{aligned}
& a_{ \pm}(\lambda)=\lambda^{d / 2} \beta_{d}\left(\alpha_{0}^{ \pm}+O\left(\lambda^{\epsilon}\right)+i O\left(\lambda^{-(d-2) \epsilon}\right)\right), \\
& b_{ \pm}(\lambda)=i \lambda^{-(d-2) / 2} \beta_{d}\left(\beta_{0}^{ \pm}+O\left(\lambda^{\epsilon}\right)+i O\left(\lambda^{d \epsilon}\right)\right) .
\end{aligned}
$$

The $O(\cdot)$ terms are real-valued and satisfy the natural derivative bounds.

Using the expansions in Lemma 3.7 an asymptotic expansion for $W(\lambda)$ for small $\lambda$ under the nonresonant condition was obtained.

Lemma 3.8 (Corollary 3.13). If 0 is not a resonance for $H$, then for all $0<\epsilon<\epsilon_{0}(d)$, we have

$$
W(\lambda)=i e^{i \pi(d-1) / 2} \lambda^{-(d-2)}\left(W_{0}+O_{\mathbb{C}}\left(\lambda^{\epsilon}\right)\right) .
$$

Here $W_{0}$ is a nonzero real constant and $O_{\mathbb{C}}\left(\lambda^{\epsilon}\right)$ is complex valued, and all terms satisfy the natural derivative bounds. We remark that the nonresonant condition is what guarantees that the constant $W_{0}$ is nonzero.

Finally, the following asymptotic expansion for the spectral measure corresponding to $H$ for small $\lambda$ was obtained.

Lemma 3.9 (Corollary 5.1 [24]). If 0 is not a resonance for $H$, then for $0<\lambda \ll 1$ and any $r, \rho \in \mathbb{R}$,

$$
\begin{aligned}
\mathfrak{J}\left[\frac{f_{+}(r, \lambda) f_{-}(\rho, \lambda)}{W(\lambda)}\right] & =O\left(\lambda^{d-1}\right) u_{0}^{+}(r, \lambda) u_{1}^{-}(\rho, \lambda) \\
+O\left(\lambda^{d-1}\right) u_{1}^{+}(r, \lambda) u_{0}^{-}(\rho, \lambda) & +O\left(\lambda^{d-1}\right) u_{0}^{+}(r, \lambda) u_{0}^{-}(\rho, \lambda) \\
& +O\left(\lambda^{d-1}\right) u_{1}^{+}(r, \lambda) u_{1}^{-}(\rho, \lambda),
\end{aligned}
$$

where the $O(\cdot)$ terms are real-valued and satisfy the natural derivative bounds.

We now turn to proving the oscillatory integral estimate Proposition 3.2 
3.2. Proof of Proposition 3.2. We recall that we wish to prove the oscillatory integral estimate

$$
\left|\int_{0}^{\infty} e^{ \pm i t \lambda} \varphi\left(2^{-j} \lambda\right) \lambda \mathfrak{I}\left[\frac{f_{+}(r, \lambda) f_{-}(\rho, \lambda)}{W(\lambda)}\right] d \lambda\right| \lesssim(\langle r\rangle\langle\rho\rangle)^{d / 2} 2^{j(d+2) / 2}\left(2^{-j}+|t|\right)^{-d / 2},
$$

for all $r>\rho$ and $t \in \mathbb{R}$. Here $H$ is the Schrödinger operator on $\mathbb{R}$

$$
H=-\frac{d^{2}}{d r^{2}}+V, \quad V(r)=\frac{d(d-4)}{4} r^{2}\langle r\rangle^{-4}+\frac{d}{2}\langle r\rangle^{-2},
$$

and $f_{ \pm}(\cdot, \lambda)$ are the Jost functions associated to $H$. We distinguish the cases $j \ll 0$ and $j \gtrsim 0$. The case $j \ll 0$ will rely heavily on the scattering theory summarized in the previous subsection.

We first consider the case $j \ll 0$ so that the integrand in the oscillatory integral is localized to small $\lambda$. We first claim that $H$ is nonresonant so that the results summarized in the previous section apply. Indeed, if 0 is a resonance of $H$, then there exists a nonzero function $f$ such that $H f=0$ and $f(r)=O\left(\langle r\rangle^{-(d-2) / 2}\right)$ as $|r| \rightarrow \infty$. This implies by the relation $-\Delta_{g}\left(\langle r\rangle^{-d / 2} f\right)=\langle r\rangle^{-d / 2} H f$ that there exists a nonzero function $u$ such that $\Delta_{g} u=0$ and $u(r)=O\left(\langle r\rangle^{-(d-1)}\right)$ as $|r| \rightarrow \infty$. Since $d \geq 2$, the maximum principle on $\mathcal{M}$ implies that $u \equiv 0$, a contradiction. Thus, 0 is not a resonance of the Schrodinger operator $H$.

The proof of Proposition 3.2 for $j \ll 0$ is split up into several lemmas. In what follows, we differentiate between the oscillatory regime and the exponential regime for the Jost solutions $f_{ \pm}(\cdot, \lambda)$. This transition occurs at $|r \lambda|=1$. Let $\chi \in C_{0}^{\infty}(\mathbb{R})$ be even with $\chi(r)=1$ for $|r| \leq 1$ and supp $\chi \subset\{|r|<2\}$. We denote the smooth cutoff $\chi(r \lambda)$ by $\chi_{[|r \lambda|<1]}$ and the smooth cutoff $(1-\chi(r \lambda))$ by $\chi_{[|r \lambda|>1]}$.

Lemma 3.10. For all $t \in \mathbb{R}$ and $r, \rho \in \mathbb{R}$,

$$
\left|\int_{0}^{\infty} e^{ \pm i t \lambda} \lambda \varphi\left(2^{-j} \lambda\right) \chi_{[|r \lambda|<1]} \chi_{[|\rho \lambda|<1]}(\langle r\rangle\langle\rho\rangle)^{-d / 2} \mathfrak{T}\left[\frac{f_{+}(r, \lambda) f_{-}(\rho, \lambda)}{W(\lambda)}\right] d \lambda\right| \lesssim 2^{j(d+2) / 2}\left(2^{-j}+|t|\right)^{-d / 2} .
$$

Proof. By Lemma 3.9 we may write

$$
\mathfrak{J}\left[\frac{f_{+}(r, \lambda) f_{-}(\rho, \lambda)}{W(\lambda)}\right]=O\left(\lambda^{d-1}\right) O\left((\langle r\rangle\langle\rho\rangle)^{d / 2}\right),
$$

where the $O(\cdot)$ terms satisfy natural derivative bounds. We write (3.4) as

$$
\left|\int_{0}^{\infty} e^{ \pm i t \lambda} a_{j}(r, \rho, \lambda) d \lambda\right| \lesssim 2^{j(d+2) / 2}\left(2^{-j}+|t|\right)^{-d / 2},
$$

where

$$
a_{j}(r, \rho, \lambda)=\lambda \varphi\left(2^{-j} \lambda\right) \chi_{[|r \lambda|<1]} \chi_{[|\rho \lambda|<1]}(\langle r\rangle\langle\rho\rangle)^{-d / 2} \mathfrak{J}\left[\frac{f_{+}(r, \lambda) f_{-}(\rho, \lambda)}{W(\lambda)}\right] .
$$

By (3.5) the function $a_{j}(r, \rho, \lambda)$ satisfies

$$
a_{j}(r, \rho, \lambda)=\varphi\left(2^{-j} \lambda\right) O\left(\lambda^{d}\right)
$$

with natural derivative bounds.

First note that if $|t| \leq 2^{-j}$, then by (3.7)

$$
\left|\int_{0}^{\infty} e^{ \pm i t \lambda} a_{j}(r, \rho, \lambda) d \lambda\right| \lesssim \int_{\left[\lambda \approx 2^{j}\right]} \lambda^{d} d \lambda \lesssim 2^{j(d+1)} \lesssim 2^{j(d+2) / 2}\left(2^{-j}+|t|\right)^{-d / 2} .
$$

We now assume that $|t| \geq 2^{-j}$. Integration by parts $d$ times and (3.7) yield

$$
\begin{aligned}
\left|\int_{0}^{\infty} e^{ \pm i t \lambda} a_{j}(r, \rho, \lambda) d \lambda\right| & =|t|^{-d}\left|\int_{0}^{\infty} e^{ \pm i t \lambda} \partial_{\lambda}^{d} a_{j}(r, \rho, \lambda) d \lambda\right| \\
& \lesssim|t|^{-d} \int_{\left[\lambda \sim 2^{j}\right]} d \lambda \\
& \lesssim|t|^{-d} 2^{j} \\
& \lesssim 2^{j(d+2) / 2}\left(2^{-j}+|t|\right)^{-d / 2} .
\end{aligned}
$$

This concludes the proof. 
We now consider the case when the integrand is supported in $|r \lambda|>1$ and $|\rho \lambda|>1$. With the convention that $f_{ \pm}(\cdot,-\lambda)=\overline{f_{ \pm}(\cdot, \lambda)}$, we remove the taking of an imaginary part in the integrand and write

$$
\begin{aligned}
\int_{0}^{\infty} e^{ \pm i t \lambda} \lambda \varphi\left(2^{-j} \lambda\right) \chi(\lambda r) \chi(\lambda \rho)(\langle r\rangle\langle\rho\rangle)^{-d / 2} \mathfrak{J}\left[\frac{f_{+}(r, \lambda) f_{-}(\rho, \lambda)}{W(\lambda)}\right] d \lambda \\
\quad=\int_{-\infty}^{\infty} e^{ \pm i t|\lambda|} \lambda \varphi\left(2^{-j} \lambda\right) \chi(\lambda r) \chi(\lambda \rho)(\langle r\rangle\langle\rho\rangle)^{-d / 2} \frac{f_{+}(r, \lambda) f_{-}(\rho, \lambda)}{W(\lambda)} d \lambda .
\end{aligned}
$$

We first consider the case $\rho<0<r$.

Lemma 3.11. For all $t \in \mathbb{R}$ and $\rho<0<r$

$$
\left|\int_{-\infty}^{\infty} e^{ \pm i t|\lambda|} \lambda \varphi\left(2^{-j} \lambda\right) \chi_{[|r \lambda|>1]} \chi_{[|\rho \lambda|>1]}(\langle r\rangle\langle\rho\rangle)^{-d / 2} \frac{f_{+}(r, \lambda) f_{-}(\rho, \lambda)}{W(\lambda)} d \lambda\right| \lesssim 2^{j(d+2) / 2}\left(2^{-j}+|t|\right)^{-d / 2} .
$$

Proof. We first note that by Lemma 3.6 and Lemma 3.8 ,

$$
\sup _{|r \lambda|>1,|\rho \lambda|>1}|\lambda|\left|\frac{f_{+}(r, \lambda) f_{-}(\rho, \lambda)}{W(\lambda)}\right| \lesssim 1
$$

This implies that

$$
\begin{aligned}
& \left|\int_{-\infty}^{\infty} e^{ \pm i t|\lambda|} \lambda \varphi\left(2^{-j} \lambda\right) \chi_{[|r \lambda|>1]} \chi_{[|\rho \lambda|>1]}(\langle r\rangle\langle\rho\rangle)^{-d / 2} \frac{f_{+}(r, \lambda) f_{-}(\rho, \lambda)}{W(\lambda)} d \lambda\right| \\
& \quad \lesssim \int_{-\infty}^{\infty} \varphi\left(2^{-j} \lambda\right) \chi_{[|r \lambda|>1]} \chi_{[|\rho \lambda|>1]}(\langle r\rangle\langle\rho\rangle)^{-d / 2} d \lambda \\
& \quad \lesssim \int_{-\infty}^{\infty} \varphi\left(2^{-j} \lambda\right) \lambda^{d} d \lambda \\
& \quad \lesssim 2^{j(d+1)}
\end{aligned}
$$

Thus, we only need to consider the case $|t| \geq 2^{-j}$.

Assume that $|t| \geq 2^{-j}$. By Lemma 3.6, we write

$$
f_{+}(r, \lambda)=e^{i \lambda r} m_{+}(r, \lambda), \quad f_{-}(\rho, \lambda)=e^{-i \lambda \rho} m_{-}(\rho, \lambda),
$$

where

$$
m_{+}(r, \lambda)=1+O\left(\lambda^{-1} r^{-1}\right), \quad r|\lambda|>1,
$$

with natural derivative bounds. A similar expression holds for $m_{-}(\rho, \lambda)$. We express (3.8) as

$$
\left|\int_{-\infty}^{\infty} e^{i|\lambda|\left( \pm t+\frac{\lambda}{|\lambda|}(r-\rho)\right)} a_{j}(r, \rho, \lambda) d \lambda\right| \lesssim 2^{j(d+2) / 2}\left(2^{-j}+|t|\right)^{-d / 2},
$$

where

$$
a_{j}(r, \rho, \lambda)=\lambda \varphi\left(2^{-j} \lambda\right) \chi_{[|r \lambda|>1]} \chi_{[|\rho \lambda|>1]}(\langle r\rangle\langle\rho\rangle)^{-d / 2} \frac{m_{+}(r, \lambda) m_{-}(\rho, \lambda)}{W(\lambda)} .
$$

By Lemma 3.8

$$
\frac{\lambda}{W(\lambda)}=O\left(\lambda^{d-1}\right),
$$

with natural derivative bounds. This fact and (3.9) imply that

$$
a_{j}(r, \rho, \lambda)=\varphi\left(2^{-j} \lambda\right) \chi_{[|r \lambda|>1]} \chi_{[|\rho \lambda|>1]} O\left(\lambda^{d-1}\right)(\langle r\rangle\langle\rho\rangle)^{-d / 2} .
$$

Note that if $|\lambda|$ is small, $|r \lambda|>1$, and $|\rho \lambda|>1$, then we have

$$
\begin{aligned}
& (\langle r\rangle\langle\rho\rangle)^{-d / 2} \lesssim\langle r-\rho\rangle^{-d / 2}, \\
& (\langle r\rangle\langle\rho\rangle)^{-d / 2} \leq \lambda^{d} .
\end{aligned}
$$


If $|t| \lesssim|r-\rho|$, then since $j \ll 0$, we have

$$
\begin{aligned}
\left|\int_{-\infty}^{\infty} e^{i|\lambda|\left( \pm t+\frac{\lambda}{\lambda \mid}(r-\rho)\right)} a_{j}(r, \rho, \lambda) d \lambda\right| & \lesssim \int_{[|\lambda| \sim 2 j]}|\lambda|^{d-1} d \lambda\langle r-\rho\rangle^{-d / 2} \\
& \lesssim 2^{d j \mid}|t|^{-d / 2} \\
& \lesssim 2^{j(d+2) / 2}\left(2^{-j}+|t|\right)^{-d / 2} .
\end{aligned}
$$

Now suppose $|t| \gg|r-\rho|$. By (3.10) and integration by parts

$$
\begin{aligned}
\left|\int_{0}^{\infty} e^{i \lambda( \pm t+(r-\rho))} a_{j}(r, \rho, \lambda) d \lambda\right| & =| \pm t+(r-\rho)|^{-d}\left|\int_{0}^{\infty} e^{i \lambda( \pm t+(r-\rho))} \partial_{\lambda}^{d} a_{j}(r, \rho, \lambda) d \lambda\right| \\
& \lesssim|t|^{-d} \int_{\left[\lambda \sim 2^{j}\right]} \lambda^{d-1} d \lambda \\
& \lesssim|t|^{-d} 2^{d j} \\
& \lesssim 2^{j(d+2) / 2}\left(2^{-j}+|t|\right)^{-d / 2} .
\end{aligned}
$$

A similar argument shows that

$$
\left|\int_{-\infty}^{0} e^{-i \lambda( \pm t-(r-\rho))} a_{j}(r, \rho, \lambda) d \lambda\right| \lesssim 2^{j(d+2) / 2}\left(2^{-j}+|t|\right)^{-d / 2} .
$$

This concludes the proof.

We now consider the case when $|r \lambda|>1$ and $|\rho \lambda|<1$ in the integrand. The case $|r \lambda|<1$ and $|\rho \lambda|>1$ can be handled similarly.

Lemma 3.12. For all $t, r \in \mathbb{R}$ and $\rho<r$

$$
\left|\int_{-\infty}^{\infty} e^{ \pm i t|\lambda|} \lambda \varphi\left(2^{-j} \lambda\right) \chi_{[|r \lambda|>1]} \chi_{[|\rho \lambda|<1]}(\langle r\rangle\langle\rho\rangle)^{-2} \frac{f_{+}(r, \lambda) f_{-}(\rho, \lambda)}{W(\lambda)} d \lambda\right| \lesssim 2^{j(d+2) / 2}\left(2^{-j}+|t|\right)^{-d / 2} .
$$

Proof. We write $f_{+}(r, \lambda)=e^{i r \lambda} m_{+}(r, \lambda)$ as before, but since $|\rho \lambda|<1$, we use the representation

$$
f_{-}(\rho, \lambda)=a_{-}(\lambda) u_{0}^{-}(\rho, \lambda)+b_{-}(\lambda) u_{1}^{-}(\rho, \lambda) .
$$

In particular, we have that

$$
f_{-}(\rho, \lambda)=O\left(\lambda^{-(d-2) / 2}\right) O\left(\langle\rho\rangle^{d / 2}\right)
$$

Now we write $(3.11$ ) as

$$
\left|\int_{-\infty}^{\infty} e^{i \mid \lambda\left( \pm t+\frac{\lambda}{(\lambda)} r\right)} a_{j}(r, \rho, \lambda) d \lambda\right| \lesssim 2^{j(d+2) / 2}\left(2^{-j}+|t|\right)^{-d / 2}
$$

where

$$
\begin{aligned}
a_{j}(r, \rho, \lambda) & =\lambda \varphi\left(2^{-j} \lambda\right) \chi_{[|r \lambda|>1]} \chi_{[|\rho \lambda|<1]}(\langle r\rangle\langle\rho\rangle)^{-d / 2} \frac{m_{+}(r, \lambda) f_{-}(\rho, \lambda)}{W(\lambda)} \\
& =\varphi\left(2^{-j}\right) \chi_{[|r \lambda|>1]} \chi_{[|\rho \lambda|<1]} O\left(\lambda^{d / 2}\right)\langle r\rangle^{-d / 2},
\end{aligned}
$$

with natural derivative bounds. As before, in the case $|t| \geq 2^{-j}$ we have

$$
\begin{aligned}
\left|\int_{0}^{\infty} e^{i \lambda( \pm t+r)} a_{j}(r, \rho, \lambda) d \lambda\right| & \lesssim \int_{\left[\lambda \sim 2^{j}\right]} \lambda^{d} d \lambda \\
& \lesssim 2^{j(d+1)} \\
& \lesssim 2^{j(d+2) / 2}\left(2^{-j}+|t|\right)^{-d / 2} .
\end{aligned}
$$

Thus, we need only consider the case that $|t| \geq 2^{-j}$. 
Suppose that $|t| \geq 2^{-j}$. If $|t| \lesssim|r|$ then

$$
\begin{aligned}
\left|\int_{0}^{\infty} e^{i \lambda( \pm t+r)} a_{j}(r, \rho, \lambda) d \lambda\right| & \lesssim \int_{\left[\lambda \simeq 2^{j}\right]} \lambda^{d / 2}|t|^{-d / 2} d \lambda \\
& \lesssim 2^{j(d+2) / 2}\left(2^{-j}+|t|\right)^{-d / 2} .
\end{aligned}
$$

If $|t| \gg|r|$, then by integration by parts

$$
\begin{aligned}
\left|\int_{0}^{\infty} e^{i \lambda( \pm t+r)} a_{j}(r, \rho, \lambda) d \lambda\right| & =| \pm t+r|^{-d}\left|\int_{0}^{\infty} e^{i \lambda( \pm t+r)} \partial_{\lambda}^{d} a_{j}(r, \rho, \lambda) d \lambda\right| \\
& \lesssim|t|^{-d} \int_{\left[\lambda \sim 2^{j}\right]} d r \\
& \lesssim|t|^{-d} 2^{j} \\
& \lesssim 2^{j(d+2) / 2}\left(2^{-j}+|t|\right)^{-d / 2},
\end{aligned}
$$

as desired. Similarly,

$$
\left|\int_{-\infty}^{0} e^{i \lambda( \pm t-r)} a_{j}(r, \rho, \lambda) d \lambda\right| \lesssim 2^{j(d+2) / 2}\left(2^{-j}+|t|\right)^{-d / 2} .
$$

This concludes the proof.

To finish proving Proposition 3.2 in the case $j \ll 0$, we need only consider the case when the integrand is supported in $|\lambda|^{-1}<\rho<r$. The case $\rho<r<-|\lambda|^{-1}$ can be dealt with in a similar fashion. We consider reflection and transmission coefficients $\alpha_{-}(\lambda), \beta_{-}(\lambda)$ defined by the relation

$$
f_{-}(\rho, \lambda)=\alpha_{-}(\lambda) f_{+}(\rho, \lambda)+\beta_{-}(\lambda) \overline{f_{+}(\rho, \lambda)}
$$

Then

$$
\begin{aligned}
W(\lambda) & =W\left(f_{-}(\cdot, \lambda), f_{+}(\cdot, \lambda)\right) \\
& =-\beta_{-}(\lambda) W\left(f_{+}(\cdot, \lambda), \overline{f_{+}(\cdot, \lambda)}\right) \\
& =-\beta_{-}(\lambda) \lim _{r \rightarrow \infty} W\left(f_{+}(r, \lambda), \overline{f_{+}(r, \lambda)}\right) \\
& =-\beta_{-}(\lambda) \lim _{r \rightarrow \infty} W\left(e^{i \lambda r}, e^{-i \lambda r}\right) \\
& =2 i \lambda \beta_{-}(\lambda) .
\end{aligned}
$$

Let $\widetilde{W}(\lambda)=W\left(f_{-}(\cdot, \lambda), \overline{f_{+}(\cdot, \lambda)}\right)$. Then similar to $W(\lambda)$ we have

$$
\begin{aligned}
\widetilde{W}(\lambda) & =\alpha_{-}(\lambda) W\left(f_{+}(\cdot, \lambda), \overline{f_{+}(\cdot, \lambda)}\right) \\
& =-2 i \lambda \alpha_{-}(\lambda) .
\end{aligned}
$$

We conclude that

$$
\begin{aligned}
& \lambda \frac{\beta_{-}(\lambda)}{W(\lambda)}=\frac{1}{2 i}, \\
& \lambda \frac{\alpha_{-}(\lambda)}{W(\lambda)}=-\frac{1}{2 i} \frac{\widetilde{W}(\lambda)}{W(\lambda)}=\text { constant }+O\left(\lambda^{\epsilon}\right),
\end{aligned}
$$

where the $O\left(\lambda^{e}\right)$ term is complex valued and satisfies natural derivative bounds. The second equality in the second line above follows from Lemma 3.7

Lemma 3.13. For all $t \in \mathbb{R}$ and $0<\rho<r$

$$
\left|\int_{-\infty}^{\infty} e^{ \pm i t|\lambda|} \lambda \varphi\left(2^{-j} \lambda\right) \chi_{[|\rho \lambda|>1]}(\langle r\rangle\langle\rho\rangle)^{-2} \frac{f_{+}(r, \lambda) f_{-}(\rho, \lambda)}{W(\lambda)} d \lambda\right| \lesssim 2^{j(d+2) / 2}\left(2^{-j}+|t|\right)^{-d / 2} .
$$


Proof. We write $f_{+}(r, \lambda)=e^{i \lambda r} m_{+}(r, \lambda)$. Then

$$
\begin{aligned}
\lambda \frac{f_{+}(r, \lambda) f_{-}(\rho, \lambda)}{W(\lambda)} & =e^{i(r+\rho) \lambda} \lambda \frac{\alpha_{-}(\lambda)}{W(\lambda)} m_{+}(r, \lambda) m_{+}(\rho, \lambda)+e^{i(r-\rho) \lambda} \lambda \frac{\beta_{-}(\lambda)}{W(\lambda)} m_{+}(r, \lambda) \overline{m_{+}(\rho, \lambda)} \\
& =e^{i(r+\rho) \lambda} O(1) m_{+}(r, \lambda) m_{+}(\rho, \lambda)+\frac{1}{2 i} e^{i(r-\rho) \lambda} m_{+}(r, \lambda) \overline{m_{+}(\rho, \lambda)}
\end{aligned}
$$

where the $O(1)$ term is complex valued and satisfies natural derivative bounds. We are thus reduced to proving the following two estimates

$$
\begin{aligned}
& \left|\int_{-\infty}^{\infty} e^{ \pm i t|\lambda|\left( \pm t+\frac{\lambda}{\lambda \mid}(r+\rho)\right)} \varphi\left(2^{-j} \lambda\right) \chi_{[|\rho \lambda|>1]}(\langle r\rangle\langle\rho\rangle)^{-d / 2} O(1) m_{+}(r, \lambda) m_{+}(\rho, \lambda) d \lambda\right| \lesssim 2^{j(d+2) / 2}\left(2^{-j}+|t|\right)^{-d / 2}, \\
& \left|\int_{-\infty}^{\infty} e^{ \pm i t|\lambda|\left( \pm t+\frac{\lambda}{\lambda \mid}(r-\rho)\right)} \varphi\left(2^{-j} \lambda\right) \chi_{[|\rho \lambda|>1]}(\langle r\rangle\langle\rho\rangle)^{-d / 2} m_{+}(r, \lambda) \overline{m_{+}(\rho, \lambda)} d \lambda\right| \lesssim 2^{j(d+2) / 2}\left(2^{-j}+|t|\right)^{-d / 2} .
\end{aligned}
$$

We now prove (3.14).

We write (3.14) as

$$
\left|\int_{-\infty}^{\infty} e^{i|\lambda|\left( \pm t+\frac{\lambda}{\lambda(\lambda)}(r+\rho)\right)} a_{j}(r, \rho, \lambda) d \lambda\right| \lesssim 2^{j(d+2) / 2}\left(2^{-j}+|t|\right)^{-d / 2} .
$$

where

$$
\begin{aligned}
a_{j}(r, \rho, \lambda) & \left.=\varphi\left(2^{-j} \lambda\right) \chi_{[|\rho \lambda|>1]}\right)(\langle r\rangle\langle\rho\rangle)^{-d / 2} O(1) m_{+}(r, \lambda) m_{+}(\rho, \lambda) \\
& =\varphi\left(2^{-j} \lambda\right) \chi_{[|\rho \lambda|>1]}(\langle r\rangle\langle\rho\rangle)^{-d / 2} O(1),
\end{aligned}
$$

with the $O(\cdot)$ term behaving like a symbol under differentiation in $\lambda$. Note that if $|t| \leq 2^{-j}$ then $|r \lambda|>1$ and $|\rho \lambda|>1$ imply that

$$
\begin{aligned}
\left|\int_{-\infty}^{\infty} e^{i|\lambda|\left( \pm t+\frac{\lambda}{\lambda \lambda \mid}(r+\rho)\right)} a_{j}(r, \rho, \lambda) d \lambda\right| & \lesssim \int_{[\lambda \sim 2 j]} \lambda^{d} d \lambda \\
& \lesssim 2^{j(d+1)} \\
& \lesssim 2^{j(d+2) / 2)}\left(2^{-j}+|t|\right)^{-d / 2} .
\end{aligned}
$$

Thus, we need only consider $|t| \geq 2^{-j}$.

Suppose that $|t| \leq 2(r+\rho)$. Then $0<\rho<r$ implies that $r \geq|t| / 4$ so that

$$
\chi_{[|\rho \lambda|>1]}(\langle r\rangle\langle\rho\rangle)^{-d / 2} \lesssim \lambda^{d / 2}|t|^{-d / 2} .
$$

Thus,

$$
\begin{aligned}
\left|\int_{-\infty}^{\infty} e^{i|\lambda|\left( \pm t+\frac{\lambda}{\lambda \mid}(r+\rho)\right)} a_{j}(r, \rho, \lambda) d \lambda\right| & \lesssim \int_{[\lambda \simeq 2 j]} \lambda^{d / 2}|t|^{-d / 2} d \lambda \\
& \lesssim 2^{j(d+2) / 2}|t|^{-d / 2} .
\end{aligned}
$$

as desired. Suppose now that $|t| \geq 2(r+\rho)$. Integration by parts yields

$$
\begin{aligned}
\left|\int_{0}^{\infty} e^{i \lambda( \pm t+(r+\rho))} a_{j}(r, \rho, \lambda) d \lambda\right| & =| \pm t+(r+\rho)|^{-d}\left|\int_{0}^{\infty} e^{i \lambda( \pm t+(r+\rho))} \partial_{\lambda}^{d} a_{j}(r, \rho, \lambda) d \lambda\right| \\
& \lesssim|t|^{-d} \int_{\left[\lambda \simeq 2^{j}\right]} \lambda^{-d} \lambda^{d} d \lambda \\
& \lesssim|t|^{-d} 2^{j} \\
& \lesssim 2^{j(d+2) / 2}\left(2^{-j}+|t|\right)^{-d / 2} .
\end{aligned}
$$

In a similar fashion, we obtain

$$
\left|\int_{-\infty}^{0} e^{-i \lambda( \pm t-(r+\rho))} a_{j}(r, \rho, \lambda) d \lambda\right| \lesssim 2^{j(d+2) / 2}|t|^{-d / 2} .
$$

This proves (3.14). The proof of (3.15) is similar and is omitted. 
We now prove Proposition 3.2 in the case $j \gtrsim 0$. This case is considerably simpler than the case $j \ll 0$ since the Jost functions $f_{ \pm}(\cdot, \lambda)$ and their Wronskian $W(\lambda)$ are to given by the free case $H=-\frac{d^{2}}{d r^{2}}$ to leading order. Indeed, we write

$$
f_{+}(r, \lambda)=e^{i r \lambda} m_{+}(r, \lambda), \quad f_{-}(\rho, \lambda)=e^{-i \rho \lambda} m_{-}(\rho, \lambda) .
$$

From [23], we have the estimates

$$
\begin{aligned}
& m_{+}(r, \lambda)=1+O\left(\lambda^{-1}\langle r\rangle^{-1}\right), \\
&\left|\partial_{\lambda}^{l} \partial_{r}^{k} m_{+}(r, \lambda)\right| \lesssim_{l, k} \lambda^{-1-l}\langle r\rangle^{-1-k}
\end{aligned}
$$

for $r \geq 0$ and $l+k>0$. Similar estimates hold for $m_{-}(\rho, \lambda)$ with $\rho \leq 0$. It is well known that $|W(\lambda)| \geq|\lambda|$ for all $\lambda$. Using the asymptotics for $m_{ \pm}(\cdot, \lambda)$, we compute the Wronskian

$$
\begin{aligned}
W(\lambda) & =W\left(f_{-}(\cdot, \lambda), f_{+}(\cdot, \lambda)\right) \\
& =m_{+}(0, \lambda)\left(m_{-}^{\prime}(0, \lambda)-i \lambda m_{-}(0, \lambda)\right)-m_{-}(0, \lambda)\left(m_{+}^{\prime}(0, \lambda)+i \lambda m_{+}(0, \lambda)\right) \\
& =-2 i \lambda+O\left(\lambda^{-1}\right),
\end{aligned}
$$

with natural derivative bounds. We also compute the Wronskian

$$
\begin{aligned}
W\left(f_{-}(\cdot, \lambda), \overline{f_{+}(\cdot, \lambda)}\right) & =m_{-}(0, \lambda)\left(\bar{m}_{+}^{\prime}(0, \lambda)-2 i \lambda \bar{m}_{+}(0, \lambda)\right)-\bar{m}_{+}(0, \lambda)\left(m_{-}^{\prime}(0, \lambda)-2 i \lambda m_{-}(0, \lambda)\right) \\
& =m_{-}(0, \lambda) \bar{m}_{+}^{\prime}(0, \lambda)-m_{-}^{\prime}(0, \lambda) \bar{m}_{+}(0, \lambda) \\
& =O\left(\lambda^{-1}\right) .
\end{aligned}
$$

with symbol character in $\lambda$. We now prove Proposition 3.2 in the case $j \gtrsim 0$.

Lemma 3.14. For all $\rho<r$

$$
\left|\int_{-\infty}^{\infty} e^{ \pm i t|\lambda|} \lambda \varphi\left(2^{-j} \lambda\right)(\langle r\rangle\langle\rho\rangle)^{-d / 2} \frac{f_{+}(r, \lambda) f_{-}(\rho, \lambda)}{W(\lambda)} d \lambda\right| \lesssim 2^{j(d+2) / 2}\left(2^{-j}+|t|\right)^{-d / 2}
$$

Proof. We first note that the fact that

$$
\sup _{r, \rho}|\lambda|\left|\frac{f_{+}(r, \lambda) f_{-}(\rho, \lambda)}{W(\lambda)}\right| \lesssim 1
$$

implies that

$$
\begin{aligned}
\left|\int_{-\infty}^{\infty} e^{ \pm i t|\lambda|} \lambda \varphi\left(2^{-j} \lambda\right)(\langle r\rangle\langle\rho\rangle)^{-d} \frac{f_{+}(r, \lambda) f_{-}(\rho, \lambda)}{W(\lambda)} d \lambda\right| & \lesssim \int_{-\infty}^{\infty} \varphi\left(2^{-j} \lambda\right)(\langle r\rangle\langle\rho\rangle)^{-d} d \lambda \\
& \lesssim 2^{j} \\
& \lesssim 2^{j(d+2) / 2} .
\end{aligned}
$$

In the last line we used $j \gtrsim 0$. Thus, we only need to consider the case $|t| \geq 2^{-j}$. We split the remainder of the proof into cases: $\rho<0<r, 0<\rho<r$, and $\rho<r<0$. By symmetry we consider only the first two.

Assume $\rho<0<r$. We write

$$
f_{+}(r, \lambda)=e^{i \lambda r} m_{+}(r, \lambda), \quad f_{-}(\rho, \lambda)=e^{-i \lambda \rho} m_{-}(\rho, \lambda)
$$

where we have for all $r \geq 0$

$$
\begin{aligned}
\left|m_{+}(r, \lambda)\right| & \lesssim 1, \\
\left|\partial_{\lambda}^{l} m_{+}(r, \lambda)\right| & \lesssim \lambda^{-1-l}, \quad l>0,
\end{aligned}
$$

with similar estimates holding for $m_{-}(\rho, \lambda)$ for $\rho \leq 0$. We express $(3.16)$ as

$$
\left|\int_{-\infty}^{\infty} e^{i|\lambda|\left( \pm t+\frac{\lambda}{\| \lambda}(r-\rho)\right)} a_{j}(r, \rho, \lambda) d \lambda\right| \lesssim 2^{j(d+2) / 2}\left(2^{-j}+|t|\right)^{-d / 2},
$$

where

$$
a_{j}(r, \rho, \lambda)=\lambda \varphi\left(2^{-j} \lambda\right)(\langle r\rangle\langle\rho\rangle)^{-d / 2} \frac{m_{+}(r, \lambda) m_{-}(\rho, \lambda)}{W(\lambda)} .
$$


By (3.17) and (3.18) we have

$$
a_{j}(r, \rho, \lambda)=\varphi\left(2^{-j} \lambda\right)(\langle r\rangle\langle\rho\rangle)^{-d / 2} O(1)
$$

with natural derivative bounds.

Suppose that $|t| \leq 2|r-\rho|$. Then either $|r| \geq|t| / 4$ or $|\rho| \geq|t| / 4$. Suppose, without loss of generality, $|r| \geq|t| / 4$. Then by (3.19) we have

$$
\begin{aligned}
\left|\int_{-\infty}^{\infty} e^{i|\lambda|\left( \pm t+\frac{\lambda}{|\lambda|}(r-\rho)\right)} a_{j}(r, \rho, \lambda) d \lambda\right| & \lesssim \int_{\left[\lambda \sim 2^{j}\right]} d \lambda(\langle r\rangle\langle\rho\rangle)^{-d / 2} \\
& \lesssim 2^{j}|t|^{-d / 2} \\
& \lesssim 2^{j(d+2) / 2}\left(2^{-j}+|t|\right)^{-d / 2}
\end{aligned}
$$

Suppose now that $|t| \geq 2|r-\rho|$. Then by (3.19) and integration by parts

$$
\begin{aligned}
\left|\int_{0}^{\infty} e^{i \lambda( \pm t+(r-\rho))} a_{j}(r, \rho, \lambda) d \lambda\right| & =| \pm t+(r-\rho)|^{-d}\left|\int_{0}^{\infty} e^{i \lambda( \pm t+(r-\rho))} \partial_{\lambda}^{d} a_{j}(r, \rho, \lambda) d \lambda\right| \\
& \lesssim|t|^{-d} \int_{[\lambda \sim 2 j]} d \lambda \\
& \lesssim|t|^{-d} 2^{j} \\
& \lesssim 2^{j(d+2) / 2}\left(2^{-j}+|t|\right)^{-d / 2},
\end{aligned}
$$

as desired. Similarly,

$$
\left|\int_{-\infty}^{0} e^{i|\lambda|( \pm t-(r-\rho))} a_{j}(r, \rho, \lambda) d \lambda\right| \lesssim 2^{j(d+2) / 2}\left(2^{-j}+|t|\right)^{-d / 2} .
$$

This concludes the case $\rho<0<r$.

We now consider the case $0<\rho<r$. In this case, we use transmission and reflection coefficients and write

$$
f_{-}(\rho, \lambda)=\alpha_{-}(\lambda) f_{+}(\rho, \lambda)+\beta_{-}(\lambda) \overline{f_{+}(\rho, \lambda)}
$$

where

$$
\begin{aligned}
& \alpha_{-}(\lambda)=\frac{W\left(f_{-}(\cdot, \lambda) \overline{f_{+}(\cdot, \lambda)}\right)}{-2 i \lambda} \\
& \beta_{-}(\lambda)=\frac{W(\lambda)}{2 i \lambda} .
\end{aligned}
$$

Then using our high energy asymptotics for $W(\lambda)$ and $W\left(f_{-}(\cdot, \lambda), \overline{f_{+}(\cdot, \lambda)}\right)$, we have for $\lambda \gtrsim 1$

$$
\lambda \frac{\alpha_{-}(\lambda)}{W(\lambda)}=O\left(\lambda^{-2}\right)=O(1), \quad \lambda \frac{\beta_{-}(\lambda)}{W(\lambda)}=O(1),
$$

with natural derivative bounds. Thus, to prove (3.16) for the case $0<\rho<r$, we are reduced to proving the bounds

$$
\begin{aligned}
& \left|\int_{-\infty}^{\infty} e^{ \pm i t \lambda \mid\left( \pm t+\frac{\lambda}{\lambda \mid}(r+\rho)\right)} \varphi\left(2^{-j} \lambda\right)(\langle r\rangle\langle\rho\rangle)^{-d / 2} O(1) m_{+}(r, \lambda) m_{+}(\rho, \lambda) d \lambda\right| \lesssim 2^{j(d+2) / 2}\left(2^{-j}+|t|\right)^{-d / 2}, \\
& \left|\int_{-\infty}^{\infty} e^{ \pm i t \mid \lambda\left( \pm t+\frac{\lambda}{\lambda \mid}(r-\rho)\right)} \varphi\left(2^{-j} \lambda\right)(\langle r\rangle\langle\rho\rangle)^{-d / 2} O(1) m_{+}(r, \lambda) \overline{m_{+}(\rho, \lambda)} d \lambda\right| \lesssim 2^{j(d+2) / 2}\left(2^{-j}+|t|\right)^{-d / 2} .
\end{aligned}
$$

We write (3.20) as

$$
\left|\int_{-\infty}^{\infty} e^{i|\lambda|\left( \pm t+\frac{\lambda}{\lambda \mid}(r+\rho)\right)} a_{j}(r, \rho, \lambda) d \lambda\right| \lesssim 2^{j(d+2) / 2}\left(2^{-j}+|t|\right)^{-d / 2}
$$

where

$$
a_{j}(r, \rho, \lambda)=\varphi\left(2^{-j} \lambda\right)(\langle r\rangle\langle\rho\rangle)^{-d / 2} O(1) m_{+}(r, \lambda) m_{+}(\rho, \lambda) .
$$


Then $a_{j}(r, \rho, \lambda)$ satisfies

$$
a_{j}(r, \rho, \lambda)=\varphi\left(2^{-j} \lambda\right)(\langle r\rangle\langle\rho\rangle)^{-d / 2} O(1),
$$

with natural derivative bounds. But now we are in the same situation as in the case $\rho<0<r$ with (3.22) replacing (3.19) and we obtain (3.20) in a similar fashion. The estimate (3.21) is obtained similarly and we omit the details. This concludes the proof of Lemma 3.14 and also Proposition 3.2 .

\section{Reduction to Higher Dimensions and the Linearized Equation}

In this section, we initiate the study of the evolution (1.6). In the first subsection, we linearize degree $n$ solutions to (1.6) around the harmonic map $Q_{n}$ and make a reduction that incorporates the extra dispersion inherent in (1.6). Our main result, Theorem 1.1, is then restated in an equivalent form which we devote the rest of this work to proving. The remaining subsections establish Strichartz estimates for the linear part of the new equation which will be used in Section 5. In what follows we use the notation from the previous section and denote the $d$-dimensional wormhole by $\mathcal{M}^{d}$.

4.1. Reduction to a Wave Equation on a $5 d$ Wormhole. We recall from the introduction that a corotational wave map on a wormhole $U: \mathbb{R} \times \mathcal{M}^{3} \rightarrow \mathbb{S}^{3}$ with topological degree $n$ is a map $U(t, r, \theta, \varphi)=(\psi(t, r), \theta, \varphi)$ such the azimuth angle $\psi=\psi(t, r)$ satisfies the Cauchy problem

$$
\begin{aligned}
& \partial_{t}^{2} \psi-\partial_{r}^{2} \psi-\frac{2 r}{r^{2}+1} \partial_{r} \psi+\frac{\sin 2 \psi}{r^{2}+1}=0, \\
& \psi(t,-\infty)=0, \quad \psi(t, \infty)=n \pi, \quad \forall t, \\
& \vec{\psi}(0)=\left(\psi_{0}, \psi_{1}\right) .
\end{aligned}
$$

The following energy is conserved along the flow

$$
\mathcal{E}(\psi)=\frac{1}{2} \int\left[\left|\partial_{t} \psi\right|^{2}+\left|\partial_{r} \psi\right|^{2}+\frac{2 \sin ^{2} \psi}{r^{2}+1}\right]\left(r^{2}+1\right) d r,
$$

and so, it is natural to take initial data $\left(\psi_{0}, \psi_{1}\right)$ in the metric space

$$
\mathcal{E}_{n}=\left\{\left(\psi_{0}, \psi_{1}\right): \mathcal{E}\left(\psi_{0}, \psi_{1}\right)<\infty, \quad \psi_{0}(-\infty)=0, \quad \psi_{0}(\infty)=n \pi\right\} .
$$

For the remainder of this work, we fix the topological degree $n \in \mathbb{N} \cup\{0\}$. We now reduce the study of the large data solutions to (4.1) to the study of large data solutions to a semilinear wave equation on a $5 d$ wormhole.

By Proposition 2.1. there exists a unique finite energy static solution $Q_{n}$ to (4.1), i.e. a solution $Q_{n} \in \mathcal{E}_{n}$ such that

$$
\partial_{r}^{2} Q_{n}+\frac{2 r}{r^{2}+1} \partial_{r} Q_{n}-\frac{\sin 2 Q_{n}}{r^{2}+1}=0
$$

To simplify notation, we write $Q$ instead of $Q_{n}$. For a solution $\psi$ to (4.1), define $\varphi$ by

$$
\psi(t, r)=Q(r)+\varphi(t, r)
$$

Then (4.1) and (4.2) imply that $\varphi$ satisfies

$$
\begin{aligned}
& \partial_{t}^{2} \varphi-\partial_{r}^{2} \varphi-\frac{2 r}{r^{2}+1} \partial_{r} \varphi+\frac{2 \cos 2 Q}{r^{2}+1} \varphi=Z(r, \varphi), \\
& \varphi(t,-\infty)=\varphi(t, \infty)=0, \quad \forall t \\
& \vec{\varphi}(0)=\left(\psi_{0}-Q, \psi_{1}\right)
\end{aligned}
$$

where

$$
Z(r, \phi)=\frac{1}{r^{2}+1}[2 \varphi-\sin 2 \varphi] \cos 2 Q+(1-\cos 2 \varphi) \sin 2 Q .
$$

The left-hand side of (4.3) has more dispersion than a free wave on $\mathcal{M}^{3}$ due to the repulsive potential

$$
\frac{2 \cos 2 Q}{r^{2}+1}=\frac{2}{r^{2}+1}+O\left(\langle r\rangle^{-6}\right)
$$


as $r \rightarrow \pm \infty$. The $O\left(\langle r\rangle^{-6}\right)$ term is due to the asymptotics of $Q$ at $\pm \infty$ (see Proposition 2.1). We now make a standard reduction that incorporates this extra dispersion. Set $\varphi=\langle r\rangle u$. Then $u$ satisfies the radial semilinear wave equation

$$
\begin{aligned}
& \partial_{t}^{2} u-\Delta_{g} u+V(r) u=N(r, u), \\
& u(t,-\infty)=u(t, \infty)=0, \quad \forall t, \\
& \vec{u}(0)=\left(u_{0}, u_{1}\right),
\end{aligned}
$$

where $-\Delta_{g}$ is the (radial) Laplace operator on $\mathcal{M}^{5}$

$$
-\Delta_{g} u=-\partial_{r}^{2} u-\frac{4 r}{r^{2}+1} \partial_{r} u,
$$

the potential is

$$
V(r)=\langle r\rangle^{-4}+2\langle r\rangle^{-2}(\cos 2 Q-1)
$$

and $N(r, u)=F(r, u)+G(r, u)$ with

$$
\begin{aligned}
& F(r, u)=2\langle r\rangle^{-3} \sin ^{2}(\langle r\rangle u) \sin 2 Q, \\
& G(r, u)=\langle r\rangle^{-3}[2\langle r\rangle u-\sin (2\langle r\rangle u)] \cos 2 Q .
\end{aligned}
$$

By Proposition 2.1, the potential $V$ is smooth and satisfies

$$
V(r)=\langle r\rangle^{-4}+O\left(\langle r\rangle^{-6}\right) .
$$

Moreover, since $Q(-r)+Q(r)=n \pi, V(r)$ is an even function. The nonlinearities $F$ and $G$ satisfy

$$
\begin{aligned}
F(r, u) & =\left(2 \sin 2 Q\langle r\rangle^{-1}\right) u^{2}+F_{0}(r, u), \\
\left|F_{0}(r, u)\right| & \lesssim\langle r\rangle^{-1} u^{4}, \\
|G(r, u)| & \lesssim|u|^{3},
\end{aligned}
$$

where the implied constants are absolute. Based on our definition of $u$ in terms of the original azimuth function $\psi$, we consider radial initial data $\left(u_{0}, u_{1}\right) \in \mathcal{H}\left(\mathbb{R} ;\left(r^{2}+1\right)^{2} d r\right)$ in (4.4). For the remainder of this section, we denote $\mathcal{H}_{0}:=\mathcal{H}\left(\mathbb{R} ;\left(r^{2}+1\right) d r\right)$ and $\mathcal{H}:=\mathcal{H}\left(\mathbb{R} ;\left(r^{2}+1\right)^{2} d r\right)$ by $\mathcal{H}$. We note that $\mathcal{H}_{0}$ is simply the space of radial functions in $\dot{H}^{1} \times L^{2}\left(\mathcal{M}^{3}\right)$ and $\mathcal{H}$ is the space of radial functions in $\dot{H}^{1} \times L^{2}\left(\mathcal{M}^{5}\right)$.

In the remainder of the paper, we work only with the ' $u$-formulation' rather than with the original azimuth angle $\psi$. The reason that a solution $\vec{\psi}(t) \in C\left(\mathbb{R} ; \mathcal{H}_{n}\right)$ to (4.1) with initial data $\left(\psi_{0}, \psi_{1}\right) \in \mathcal{E}_{n}$ yields a solution $\vec{u}(t) \in C(\mathbb{R} ; \mathcal{H})$ with initial data $\left(u_{0}, u_{1}\right)=\langle r\rangle^{-1}\left(\psi_{0}-Q, \psi_{1}\right) \in \mathcal{H}$ and vice versa is as follows. The only fact that needs to be checked is that

$$
\|\vec{u}\|_{\mathcal{H}} \simeq\|\vec{\psi}-(Q, 0)\|_{\mathcal{H}_{0}}
$$

Set $\varphi=\psi-Q=\langle r\rangle$. Then

$$
\partial_{r} \varphi=\langle r\rangle \partial_{r} u+\frac{r}{\langle r\rangle^{2}} u .
$$

We note that we have the following Hardy's inequalities

$$
\begin{aligned}
\int|\varphi|^{2} d r & \lesssim \int\left|\partial_{r} \varphi\right|^{2}\left(r^{2}+1\right) d r \\
\int|u|^{2}\left(r^{2}+1\right) d r & \lesssim \int\left|\partial_{r} u\right|^{2}\left(r^{2}+1\right)^{2} d r .
\end{aligned}
$$

These estimates follow easily from integration by parts and the Strauss estimates

$$
\begin{aligned}
& |\varphi(r)| \lesssim\langle r\rangle^{-1 / 2}\left(\int\left|\partial_{r} \varphi\right|^{2}\left(r^{2}+1\right) d r\right)^{1 / 2}, \\
& |u(r)| \lesssim\langle r\rangle^{-3 / 2}\left(\int\left|\partial_{r} u\right|^{2}\left(r^{2}+1\right)^{2} d r\right)^{1 / 2} .
\end{aligned}
$$


The Strauss estimates are a simple consequence of the fundamental theorem of calculus. The two Hardy's inequalities and (4.12) imply (4.11). Hence, the two Cauchy problems (4.1) and (4.4) are equivalent.

The equivalent $u$-formulation of our main result, Theorem 1.1, is the following.

Theorem 4.1. For any initial data $\left(u_{0}, u_{1}\right) \in \mathcal{H}$, there exists a unique global solution $\vec{u}(t) \in C(\mathbb{R} ; \mathcal{H})$ to (4.4) which scatters to free waves on $\mathcal{M}^{5}$, i.e. there exist solutions $v_{L}^{ \pm}$to

$$
\partial_{t}^{2} v-\partial_{r}^{2} v-\frac{4 r}{r^{2}+1} \partial_{r} v=0, \quad(t, r) \in \mathbb{R} \times \mathbb{R},
$$

such that

$$
\lim _{t \rightarrow \pm \infty}\left\|\vec{u}(t)-\vec{v}_{L}^{ \pm}(t)\right\|_{\mathcal{H}}=0
$$

The remainder of this work is devoted to proving Theorem 4.1 In order to study the nonlinear evolution (4.4), we will need Strichartz estimates for the linear operator $\partial_{t}^{2}-\Delta_{g}+V$ where $V$ is as in (4.5).

4.2. Strichartz Estimates for the Linearized Operator. The goal of this subsection is to prove Strichartz estimates for radial solutions to the free wave equation on $\mathcal{M}^{5}$ perturbed by a radial potential $V=V(r)$

$$
\begin{aligned}
& \partial_{t}^{2} u-\Delta_{g} u+V u=F, \quad(t, r) \in I \times \mathbb{R}, \\
& \vec{u}(0)=\left(u_{0}, u_{1}\right) .
\end{aligned}
$$

The particular case we are interested in is the case that the potential $V$ is given by

$$
V(r)=\langle r\rangle^{-4}+2\langle r\rangle^{-2}(\cos 2 Q-1),
$$

where $Q$ is the unique harmonic map of degree $n$. The Strichartz estimates we establish will be used in the next section to study the nonlinear evolution (4.4). We recall from Section 3 that we say that a triple $(a, b, \gamma)$ is admissible for $M^{5}$ if

$$
p>2, q \geq 2, \quad \frac{1}{p}+\frac{5}{q}=\frac{5}{2}-\gamma, \quad \frac{1}{p} \leq 1-\frac{2}{q} .
$$

The main result of this subsection is the following.

Proposition 4.2. Let $V \in C^{\infty}(\mathbb{R})$ be even such that

$$
\left|V^{(j)}(r)\right| \lesssim_{j}\langle r\rangle^{-4-j}
$$

for all $r \in \mathbb{R}$. Assume that $-\Delta_{g}+V$ has no point spectrum (when restricted to radial functions) and that 0 is not a resonance of the Schrödinger operator on the line given by $-\frac{d^{2}}{d r^{2}}+2\langle r\rangle^{-2}+V(r)$. Let $(p, q, \gamma)$ and $(a, b, \rho)$ be two admissible triples for $\mathcal{M}^{5}$. Then any radial solution $u$ to (4.14) satisfies

$$
\left\|\left.|| \nabla\right|^{1-\gamma} u\right\|_{L_{t}^{p} L_{x}^{q}(I)}+\left\||\nabla|^{-\gamma} \partial_{t} u\right\|_{L_{t}^{p} L_{x}^{q}(I)} \lesssim\|\vec{u}(0)\|_{\mathcal{H}}+\left\|\left|\left\|\left.\nabla\right|^{\rho} F\right\|_{L_{t}^{a^{\prime}} L_{x}^{b^{\prime}(I)}} .\right.\right.
$$

Proof. The proof is based on arguments in Section 5 of [18]. By standard TT $T^{*}$ arguments and Minkowski's inequality (c.f. [26] or [27]), we only need to consider the case $F=0$. As we will see, the proof of Proposition 4.2 reduces to proving certain local energy estimates. Indeed, define

$$
A=\sqrt{-\Delta_{g}} .
$$

Note that

$$
\|A f\|_{L^{2}}^{2}=\left(A^{2} f, f\right)_{L^{2}}=\left(-\Delta_{g} f, f\right)_{L^{2}}=\|\nabla f\|_{L^{2}}^{2} .
$$

For a solution $u$ to (4.14), define

$$
w(t)=A u(t)+i \partial_{t} u(t)
$$

Then by (4.17),

$$
\|w(t)\|_{L^{2}}=\|\vec{u}(t)\|_{\mathcal{H}}
$$


and $w$ satisfies

$$
\begin{aligned}
& i \partial_{t} w=A w+V u, \quad(t, r) \in I \times \mathbb{R}, \\
& w(0)=A u_{0}+i u_{1} .
\end{aligned}
$$

By Duhamel's principle, (4.20) implies that

$$
w(t)=e^{-i t A} w(0)-i \int_{0}^{t} e^{-i(t-s) A} V u(s) d s .
$$

The Strichartz estimates (4.16) can be restated as

$$
\|P w\|_{X} \leq\|w(0)\|_{L^{2}}
$$

where $P:=A^{-1} \mathfrak{R}$ and $\|\cdot\|_{X}:=\left\||\nabla|^{-\gamma} \nabla_{t, x} \cdot\right\|_{L_{t}^{p} L_{x}^{q}(I)}$. By Proposition 3.1 ,

$$
\left\|P e^{-i t A} w(0)\right\|_{X} \lesssim\|w(0)\|_{L^{2}} .
$$

Thus,

$$
\|P w\|_{X} \lesssim\|w(0)\|_{L^{2}}+\left\|P \int_{0}^{t} e^{-i(t-s) A} V u(s) d s\right\|_{X} .
$$

By the Christ-Kiselev lemma, to bound the second term above, it suffices to show that

$$
\left\|P \int_{-\infty}^{\infty} e^{-i(t-s) A} V u(s) d s\right\|_{X} \lesssim\|w(0)\|_{L^{2}} .
$$

To prove (4.23), we write $V=V_{1} V_{2}$ were each factor $V_{j}$ is even and satisfies $\left|V_{j}(r)\right| \lesssim\langle r\rangle^{-2}$. Then

$$
\left\|P \int_{-\infty}^{\infty} e^{-i(t-s) A} V u(s) d s\right\|_{X} \lesssim\|K\|_{L_{t, x}^{2} \rightarrow X}\left\|V_{2} u\right\|_{X}
$$

where

$$
K F(t):=P \int_{-\infty}^{\infty} e^{-i(t-s) A} V_{1} F(s) d s
$$

If $F \in L_{t, x}^{2}$, then by (4.22)

$$
\begin{aligned}
\|K F\|_{X} & \leq\left\|P e^{-i t A}\right\|_{L_{x}^{2} \rightarrow X}\left\|\int_{-\infty}^{\infty} e^{i s A} V_{1} F(s) d s\right\|_{L_{x}^{2}} \\
& \lesssim\left\|\int_{-\infty}^{\infty} e^{i s A} V_{1} F(s) d s\right\|_{L_{x}^{2}} .
\end{aligned}
$$

We now wish to show that

$$
\left\|\int_{-\infty}^{\infty} e^{i s A} V_{1} F(s) d s\right\|_{L_{x}^{2}} \lesssim\|F\|_{L_{t, x}^{2}} .
$$

By duality, this estimate is equivalent to the local energy estimate

$$
\left\|V_{1} e^{-i t A} \varphi\right\|_{L_{t, x}^{2}} \lesssim\|\varphi\|_{L_{x}^{2}}
$$

Thus, by (4.24), the proof of Proposition 4.2 is reduced to proving the local energy estimates

$$
\begin{aligned}
\left\|V_{1} e^{-i t A} \varphi\right\|_{L_{t, x}^{2}} & \lesssim\|\varphi\|_{L_{x}^{2},} \\
\left\|V_{2} u\right\|_{L_{t, x}^{2}} & \lesssim\|\vec{u}(0)\|_{\mathcal{H}} .
\end{aligned}
$$

To prove (4.25) and (4.26), we first eliminate the weight $\langle r\rangle^{4}$ inherent in them. Consider the isomorphism $\phi \mapsto f:=\langle r\rangle^{2} \varphi$ from $L^{2}\left(\mathcal{M}^{5}\right)$ (restricted to radial functions) to $L^{2}(\mathbb{R})$. Define the following Schrödinger 
operators on $\mathbb{R}$ by

$$
\begin{gathered}
H_{0}:=-\frac{d^{2}}{d r^{2}}+\frac{2}{r^{2}+1}, \\
H:=H_{0}+V:=-\frac{d^{2}}{d r^{2}}+\frac{2}{r^{2}+1}+V(r) .
\end{gathered}
$$

Then

$$
\begin{aligned}
H_{0} & =\langle r\rangle^{2}\left(-\Delta_{g}\right)\langle r\rangle^{-2}, \\
H & =\langle r\rangle^{2}\left(-\Delta_{g}+V\right)\langle r\rangle^{-2} .
\end{aligned}
$$

Thus, from (4.28), we see that (4.25) is equivalent to the estimate

$$
\left\|V_{1} e^{-i t \sqrt{H_{0}}} f\right\|_{L_{t, r}^{2}(\mathbb{R} \times \mathbb{R})} \lesssim\|f\|_{L^{2}(\mathbb{R})} .
$$

We claim that there exist a distorted Fourier basis $\left\{\theta_{0}\left(r, \lambda^{2}\right), \phi_{0}\left(r, \lambda^{2}\right)\right\}$ that satisfies

$$
\begin{array}{cl}
H_{0} \theta_{0}\left(r, \lambda^{2}\right)=\lambda^{2} \theta_{0}\left(r, \lambda^{2}\right), & H_{0} \phi_{0}\left(r, \lambda^{2}\right)=\lambda^{2} \phi_{0}\left(r, \lambda^{2}\right), \\
\theta_{0}\left(0, \lambda^{2}\right)=1, & \phi_{0}\left(0, \lambda^{2}\right)=0, \\
\theta_{0}^{\prime}\left(0, \lambda^{2}\right)=0, & \phi_{0}^{\prime}\left(0, \lambda^{2}\right)=1,
\end{array}
$$

and positive measures $\rho_{0,1}(d \lambda)=\omega_{0,1}(\lambda) d \lambda$ and $\rho_{0,2}(d \lambda)=\omega_{0,2}(\lambda) d \lambda$ such that if we define

$$
\hat{f_{0,1}}(\lambda):=\int \theta_{0}\left(r, \lambda^{2}\right) f(r) d r, \quad \hat{f_{0,2}}(\lambda):=\int \phi_{0}\left(r, \lambda^{2}\right) f(r) d r, \quad f \in L^{2}(\mathbb{R}),
$$

then

$$
\begin{aligned}
& f(r)=\int_{0}^{\infty} \theta_{0}\left(r, \lambda^{2}\right) \hat{f_{0,1}}(\lambda) \rho_{0,1}(d \lambda)+\int_{0}^{\infty} \phi_{0}\left(r, \lambda^{2}\right) \hat{f_{0,2}}(\lambda) \rho_{0,2}(d \lambda), \\
&\|f\|_{L^{2}(\mathbb{R})}^{2}=\int_{0}^{\infty}\left|\hat{f_{0,1}}(\lambda)\right|^{2} \rho_{0,1}(d \lambda)+\int_{0}^{\infty}\left|\hat{f_{0,2}}(\lambda)\right|^{2} \rho_{0,2}(d \lambda), \\
& \sup _{r \in \mathbb{R}, \lambda>0}\left(\frac{1+\lambda^{2}\langle r\rangle^{2}}{\lambda^{2}\langle r\rangle^{2}}\right)\left[\left|\theta_{0}\left(r, \lambda^{2}\right)\right|^{2} \omega_{0,1}(\lambda)+\left|\phi_{0}\left(r, \lambda^{2}\right)\right|^{2} \omega_{0,2}(\lambda)\right]<\infty .
\end{aligned}
$$

The proof of this claim is postponed until the next subsection. Assuming the claim, we can easily establish (4.29). Indeed, since $H_{0} \mapsto \lambda^{2}$ on the Fourier side, (4.29) can be rewritten as

$$
\int\left\|V_{1}(r)\left[\int_{0}^{\infty} e^{-i t \lambda} \theta_{0}\left(r, \lambda^{2}\right) \hat{f}_{0,1}(\lambda) \rho_{0,1}(d \lambda)+\int_{0}^{\infty} e^{-i t \lambda} \phi_{0}\left(r, \lambda^{2}\right) \hat{f_{0,2}}(\lambda) \rho_{0,2}(d \lambda)\right]\right\|_{L^{2}(\mathbb{R})}^{2} d t \lesssim\|f\|_{L^{2}(\mathbb{R})}^{2} .
$$

Expanding and carrying out the $t$-integration, the left hand side of (4.34) becomes

$$
\begin{aligned}
\int V_{1}^{2}(r) & {\left[\int_{0}^{\infty} \int_{0}^{\infty} \delta(\lambda-\mu) \theta_{0}\left(r, \lambda^{2}\right) \theta_{0}\left(r, \mu^{2}\right) \hat{f_{0,1}}(\lambda) \overline{\hat{f}_{0,1}(\mu)} \rho_{0,1}(d \lambda) \rho_{0,1}(d \mu)\right.} \\
& \left.+\int_{0}^{\infty} \int_{0}^{\infty} \delta(\lambda-\mu) \phi_{0}\left(r, \lambda^{2}\right) \phi_{0}\left(r, \mu^{2}\right) \hat{f_{0,2}}(\lambda) \overline{\hat{f}_{0,2}(\mu)} \rho_{0,2}(d \lambda) \rho_{0,2}(d \mu)\right] d r \\
& =\int V_{1}^{2}(r)\left[\int_{0}^{\infty}\left|\hat{f}_{0,1}(\lambda)\right|^{2}\left|\theta_{0}\left(r, \lambda^{2}\right)\right|^{2} \omega_{0,1}^{2}(\lambda) d \lambda+\int_{0}^{\infty}\left|\hat{f_{0,2}}(\lambda)\right|^{2}\left|\phi_{0}\left(r, \lambda^{2}\right)\right|^{2} \omega_{0,2}^{2}(\lambda) d \lambda\right] d r .
\end{aligned}
$$

We remark here that no cross terms involving $\theta_{0}\left(r, \lambda^{2}\right) \phi_{0}\left(r, \mu^{2}\right)$ appeared when expanding since $V_{1}^{2}(r) \theta_{0}\left(r, \lambda^{2}\right) \phi_{0}\left(r, \mu^{2}\right)$ is an odd function of $r$ by (4.30) and our assumption that $V(r)$ is even. By (4.33) and 
(4.32), we conclude that

$$
\begin{aligned}
(4.35) & \lesssim \int V_{1}^{2}(r)\left[\int_{0}^{\infty}\left|\hat{f_{0,1}}(\lambda)\right|^{2} \omega_{0,1}(\lambda) d \lambda+\int_{0}^{\infty}\left|\hat{f_{0,2}}(\lambda)\right|^{2} \omega_{0,2}(\lambda) d \lambda\right] d r \\
& =\|f\|_{L^{2}(\mathbb{R})}^{2} \int V_{1}^{2}(r) d r \\
& \lesssim\|f\|_{L^{2}(\mathbb{R})}^{2} .
\end{aligned}
$$

This proves (4.34) which proves (4.29) as desired.

The proof of (4.26) is very similar and we sketch the details. As in the case for $H_{0}$, we claim that there exist a distorted Fourier basis $\left\{\theta\left(r, \lambda^{2}\right), \phi\left(r, \lambda^{2}\right)\right\}$ that satisfies

$$
\begin{array}{cl}
H \theta\left(r, \lambda^{2}\right)=\lambda^{2} \theta\left(r, \lambda^{2}\right), & H \phi\left(r, \lambda^{2}\right)=\lambda^{2} \phi\left(r, \lambda^{2}\right), \\
\theta\left(0, \lambda^{2}\right)=1, & \phi\left(0, \lambda^{2}\right)=0, \\
\theta^{\prime}\left(0, \lambda^{2}\right)=0, & \phi^{\prime}\left(0, \lambda^{2}\right)=1,
\end{array}
$$

and positive measures $\rho_{1}(d \lambda)=\omega_{1}(\lambda) d \lambda$ and $\rho_{2}(d \lambda)=\omega_{2}(\lambda) d \lambda$ such that if we define

$$
\hat{f_{1}}(\lambda):=\int \theta\left(r, \lambda^{2}\right) f(r) d r, \quad \hat{f_{2}}(\lambda):=\int \phi\left(r, \lambda^{2}\right) f(r) d r, \quad f \in L^{2}(\mathbb{R})
$$

then

$$
\begin{aligned}
& f(r)=\int_{0}^{\infty} \theta\left(r, \lambda^{2}\right) \hat{f_{1}}(\lambda) \rho_{1}(d \lambda)+\int_{0}^{\infty} \phi\left(r, \lambda^{2}\right) \hat{f_{2}}(\lambda) \rho_{2}(d \lambda), \\
&\|f\|_{L^{2}(\mathbb{R})}^{2}=\int_{0}^{\infty}\left|\hat{f_{1}}(\lambda)\right|^{2} \rho_{1}(d \lambda)+\int_{0}^{\infty}\left|\hat{f_{2}}(\lambda)\right|^{2} \rho_{2}(d \lambda), \\
& \sup _{r \in \mathbb{R}, \lambda>0}\left(\frac{1+\lambda^{2}\langle r\rangle^{2}}{\lambda^{2}\langle r\rangle^{2}}\right)\left[\left|\theta\left(r, \lambda^{2}\right)\right|^{2} \omega_{1}(\lambda)+\left|\phi\left(r, \lambda^{2}\right)\right|^{2} \omega_{2}(\lambda)\right]<\infty .
\end{aligned}
$$

Again, the proof of this claim is postponed until the next subsection. We remark that it is in proving (4.36), (4.37), and especially (4.38) that the spectral assumptions are crucial. By (4.28) and (4.36), we see that (4.26) follows from showing

$$
\begin{aligned}
\int \| V_{2}(r)[ & \int_{0}^{\infty}\left(\cos (t \lambda) \hat{f}_{1}(\lambda)+\lambda^{-1} \sin (t \lambda) \hat{g}_{1}(\lambda)\right) \theta\left(r, \lambda^{2}\right) \rho_{0,1}(d \lambda) \\
& \left.+\int_{0}^{\infty}\left(\cos (t \lambda) \hat{f}_{2}(\lambda)+\lambda^{-1} \sin (t \lambda) \hat{g}_{2}(\lambda)\right) \phi\left(r, \lambda^{2}\right) \rho_{2}(d \lambda)\right]\left\|_{L^{2}(\mathbb{R})}^{2} d t \lesssim\right\|(\sqrt{H} f, g) \|_{L^{2}(\mathbb{R})}^{2}
\end{aligned}
$$

Assume that $g=0$. Then, as in the case for $H_{0}$, the left side of (4.39) becomes after expanding and integrating in $t$

$$
\begin{aligned}
& \int V_{2}^{2}(r)\left[\int_{0}^{\infty}\left|\hat{f_{1}}(\lambda)\right|^{2} \cos ^{2}(t \lambda)\left|\theta\left(r, \lambda^{2}\right)\right|^{2} \omega_{1}^{2}(\lambda) d \lambda+\int_{0}^{\infty}\left|\hat{f_{2}}(\lambda)\right|^{2} \cos ^{2}(t \lambda)\left|\phi\left(r, \lambda^{2}\right)\right|^{2} \omega_{2}^{2}(\lambda) d \lambda\right] d r \\
& \quad \lesssim \int V_{2}^{2}(r)\langle r\rangle^{2}\left[\int_{0}^{\infty} \lambda^{2}\left|\hat{f_{1}}(\lambda)\right|^{2} \omega_{1}(\lambda) d \lambda+\int_{0}^{\infty} \lambda^{2}\left|\hat{f}_{2}(\lambda)\right|^{2} \omega_{2}(\lambda) d \lambda\right] d r \\
& \quad=\|\sqrt{H} f\|_{L^{2}(\mathbb{R})}^{2} \int V_{2}^{2}(r)\langle r\rangle^{2} d r \\
& \quad \lesssim \| \sqrt{(} H) f \|_{L^{2}(\mathbb{R})}^{2} .
\end{aligned}
$$

The case $g=0$ is handled similarly. This establishes (4.39) which proves (4.26). This completes the proof of Proposition 4.2 modulo the proofs of the claims about the distorted Fourier bases. We address this in the next subsection. 
4.3. The Distorted Fourier Transform. In this subsection, we prove the technical statements about the distorted Fourier bases for $H_{0}$ and $H$ used in the previous section.

Proposition 4.3. Let $H=-\frac{d^{2}}{d r^{2}}+V(r)$ be a Schrödinger operator on the line where $V \in C^{\infty}(\mathbb{R})$ is even and

$$
V(r)=\frac{2}{r^{2}}+O\left(r^{-3}\right)
$$

as $r \rightarrow \pm \infty$ with natural derivative bounds. Assume that $H$ has no point spectrum and that 0 is not a resonance of $H$. Then there exist a distorted Fourier basis $\left\{\theta\left(r, \lambda^{2}\right), \phi\left(r, \lambda^{2}\right)\right\}$ that satisfies

$$
\begin{array}{cl}
H \theta\left(r, \lambda^{2}\right)=\lambda^{2} \theta\left(r, \lambda^{2}\right), & H \phi\left(r, \lambda^{2}\right)=\lambda^{2} \phi\left(r, \lambda^{2}\right), \\
\theta\left(0, \lambda^{2}\right)=1, & \phi\left(0, \lambda^{2}\right)=0, \\
\theta^{\prime}\left(0, \lambda^{2}\right)=0, & \phi^{\prime}\left(0, \lambda^{2}\right)=1,
\end{array}
$$

and positive measures $\rho_{1}(d \lambda)=\omega_{1}(\lambda) d \lambda$ and $\rho_{2}(d \lambda)=\omega_{2}(\lambda) d \lambda$ such that if we define

$$
\hat{f}_{1}(\lambda):=\int \theta\left(r, \lambda^{2}\right) f(r) d r, \quad \hat{f}_{2}(\lambda):=\int \phi\left(r, \lambda^{2}\right) f(r) d r, \quad f \in L^{2}(\mathbb{R}),
$$

then

$$
\begin{aligned}
f(r) & =\int_{0}^{\infty} \theta\left(r, \lambda^{2}\right) \hat{f_{1}}(\lambda) \rho_{1}(d \lambda)+\int_{0}^{\infty} \phi\left(r, \lambda^{2}\right) \hat{f_{2}}(\lambda) \rho_{2}(d \lambda), \\
\|f\|_{L^{2}(\mathbb{R})}^{2} & =\int_{0}^{\infty}\left|\hat{f_{1}}(\lambda)\right|^{2} \rho_{1}(d \lambda)+\int_{0}^{\infty}\left|\hat{f_{2}}(\lambda)\right|^{2} \rho_{2}(d \lambda) \\
\sup _{r \in \mathbb{R}, \lambda>0} & \left(\frac{1+\lambda^{2}\langle r\rangle^{2}}{\lambda^{2}\langle r\rangle^{2}}\right)\left[\left|\theta\left(r, \lambda^{2}\right)\right|^{2} \omega_{1}(\lambda)+\left|\phi\left(r, \lambda^{2}\right)\right|^{2} \omega_{2}(\lambda)\right]<\infty .
\end{aligned}
$$

Many of the statements made in Proposition 4.3 follow from basic Weyl-Titchmarsch theory for Schrödinger operators on the line. We recall these basic facts now (see Section 2 of [10] for a thorough discussion). Let $H=-\frac{d}{d r^{2}}+V$ with $V \in L^{\infty}(\mathbb{R})$ (much less is needed) such that $H$ is in the limit point case at $\pm \infty$. We define $\theta(r, z), \phi(r, z)$ to be the fundamental system of solutions to

$$
H f(r)=z f(r), \quad z \in \mathbb{C},
$$

such that

$$
\begin{aligned}
\theta(0, z)=1, & \phi(0, z)=0 \\
\theta^{\prime}(0, z)=0, & \phi^{\prime}(0, z)=1
\end{aligned}
$$

By (4.45), the Wronskian is computed

$$
W(\theta(\cdot, z), \phi(\cdot, z))=1 .
$$

The condition that $H$ is in the limit point case at $\pm \infty$ implies that for $z \in \mathbb{C} \backslash \mathbb{R}$ there exist unique solutions $\psi_{ \pm}(r, z)$ to $H f=z f$ that satisfy

$$
\begin{aligned}
\psi_{ \pm}(\cdot, z) & \in L^{2}([0, \pm \infty)) \\
\psi_{ \pm}(0, z) & =1
\end{aligned}
$$

The condition at $r=0$ implies that

$$
\psi_{ \pm}(r, z)=\theta(r, z)+m_{ \pm}(z) \phi(r, z)
$$

where $m_{ \pm}(z)=W\left(\theta(\cdot, z), \psi_{ \pm}(\cdot, z)\right)$ and

$$
W\left(\psi_{+}(\cdot, z), \psi_{-}(\cdot, z)\right)=m_{-}(z)-m_{+}(z) .
$$

The functions $m_{ \pm}(z)$ can be shown to be Herglotz functions $\left(\mathfrak{J} z>0 \Longrightarrow \mathfrak{J} m_{ \pm}(z)>0\right)$ and are referred to as the Weyl-Titchmarsch functions. The associated Weyl-Titchmarsch matrix

$$
M(z):=\left[\begin{array}{cc}
\frac{1}{m_{-}(z)-m_{+}(z)} & \frac{1}{2} \frac{m_{-}(z)+m_{+}(z)}{m_{-}(z)-m_{+}(z)} \\
\frac{1}{2} \frac{m_{-}(z)+m_{+}(z)}{m_{-}(z)-m_{+}(z)} & \frac{m_{-}(z) m_{+}(z)}{m_{-}(z)-m_{+}(z)}
\end{array}\right]
$$


is a Herglotz matrix. Thus, there exists a nonnegative $2 \times 2$ matrix-valued measure $\Omega(d \lambda)$ such that

$$
M(z)=C+\int_{\mathbb{R}}\left[\frac{1}{\lambda-z}-\frac{\lambda}{1+\lambda^{2}}\right] \Omega(d \lambda),
$$

where

$$
C^{*}=C, \quad \int \frac{\|\Omega(d \lambda)\|}{1+\lambda^{2}}<\infty
$$

The measure $\Omega(d \lambda)$ is computed via

$$
\Omega\left(\left(\lambda_{1}, \lambda_{2}\right]\right)=\frac{1}{\pi} \lim _{\delta \rightarrow 0^{+}} \lim _{\epsilon \rightarrow 0^{+}} \int_{\lambda_{1}+\delta}^{\lambda_{2}+\delta} \mathfrak{J} M(\lambda+i \epsilon) d \lambda .
$$

A consequence of Weyl-Titchmarsch theory is that we have the following distorted Fourier representation for $H$.

Proposition 4.4. Let $f, g \in C_{0}^{\infty}(\mathbb{R}), F \in C(\mathbb{R}) \cap L^{\infty}(\mathbb{R})$. Let $E(d \lambda)$ denote the spectral measure for $H$. Define

$$
\hat{f_{1}}(\lambda):=\int \theta(r, \lambda) f(r) d r, \quad \hat{f_{2}}(\lambda):=\int \phi(r, \lambda) f(r) d r,
$$

and

$$
\hat{f}(\lambda)=\left(\hat{f_{1}}(\lambda), \hat{f}_{2}(\lambda)\right)^{T}
$$

Then

$$
\left(f, F(H) E\left(\left(\lambda_{1}, \lambda_{2}\right]\right) g\right)_{L^{2}(\mathbb{R})}=\int_{\left(\lambda_{1}, \lambda_{2}\right]} \hat{f}(\lambda)^{T} \Omega(d \lambda) \overline{\hat{g}(\lambda)} F(\lambda) .
$$

For the free case $V=0$, we have the following explicit expressions:

$$
\begin{gathered}
\theta(r, z)=\cos \left(r z^{1 / 2}\right), \quad \phi(r, z)=\frac{\sin \left(r z^{1 / 2}\right)}{z^{1 / 2}}, \\
\psi_{ \pm}(r, z)=e^{ \pm i r z^{1 / 2}}, \quad m_{ \pm}(z)= \pm i z^{1 / 2} \\
\Omega(d \lambda)=\frac{1}{2 \pi} \chi_{(0, \infty)}(\lambda)\left[\begin{array}{cc}
\lambda^{-1 / 2} & 0 \\
0 & \lambda^{1 / 2}
\end{array}\right] .
\end{gathered}
$$

This leads to the usual Fourier transform on the line.

Proof of Proposition 4.3 The decay of $V$ at $\pm \infty$ implies that $H=-\frac{d^{2}}{d r^{2}}+V$ is in the limit point case at $\pm \infty$ (see [10]). The decay of $V$ and the assumption that $H$ has no point spectrum imply that $\sigma(H)=[0, \infty)$ and that the spectrum is purely absolutely continuous. By Proposition 4.4, this implies that the matrix valued measure $\Omega(d \lambda)$ is supported in $[0, \infty)$. Since $V$ is even, we have by (4.41)

$$
\theta(-r, \lambda)=\theta(r, \lambda), \quad \phi(-r, \lambda)=-\phi(r, \lambda), \quad \psi_{-}(r, \lambda)=\psi_{+}(-r, \lambda),
$$

so that $m_{-}(\lambda)=-m_{+}(\lambda)$. We recall from the previous section that the Jost solutions $f_{ \pm}(r, \lambda)$ are the unique solutions to $H f=\lambda^{2} f$ such that $f_{ \pm}(r, \lambda) \sim e^{ \pm i r \lambda}$ as $r \rightarrow \pm \infty$, and that for $\lambda \neq 0, W\left(f_{+}(\cdot, \lambda), f_{-}(\cdot, \lambda)\right) \neq 0$. Then

$$
\begin{aligned}
f_{-}(r, \lambda) & =f_{+}(-r, \lambda), \\
W\left(f_{+}(\cdot, \lambda), f_{-}(\cdot, \lambda)\right) & =-2 f_{+}(0, \lambda) f_{+}^{\prime}(0, \lambda), \\
\psi_{+}\left(r, \lambda^{2}\right) & =\frac{f_{+}(r, \lambda)}{f_{+}(0, \lambda)} \\
m_{+}\left(\lambda^{2}\right) & =\frac{f_{+}^{\prime}(0, \lambda)}{f_{+}(0, \lambda)} .
\end{aligned}
$$


The matrix (4.47) satisfies

$$
\begin{aligned}
M\left(\lambda^{2}\right) & =\left[\begin{array}{cc}
-\frac{1}{2} \frac{f_{+}(0, \lambda)}{f_{+}^{\prime}(0, \lambda)} & 0 \\
0 & -\frac{1}{2} \frac{f_{+}^{\prime}(0, \lambda)}{f_{+}(0, \lambda)}
\end{array}\right] \\
& =\left[\begin{array}{cc}
\frac{1}{2} \frac{W\left(f_{+}(\cdot, \lambda), \phi\left(\cdot, \lambda^{2}\right)\right)}{W\left(f_{+}(\cdot, \lambda), \theta\left(\cdot, \lambda^{2}\right)\right)} & 0 \\
0 & -\frac{1}{2} \frac{W\left(f_{+}(\cdot, \lambda), \theta\left(\cdot, \lambda^{2}\right)\right)}{W\left(f_{+}(\cdot, \lambda), \phi\left(\cdot, \lambda^{2}\right)\right)}
\end{array}\right]
\end{aligned}
$$

Thus,

$$
\Omega\left(d \lambda^{2}\right)=\left[\begin{array}{cc}
\rho_{1}(d \lambda) & 0 \\
0 & \rho_{2}(d \lambda)
\end{array}\right]
$$

where

$$
\begin{aligned}
& \rho_{1}(d \lambda):=\frac{1}{\pi} \lambda \mathfrak{J}\left[\frac{W\left(f_{+}(\cdot, \lambda), \phi\left(\cdot, \lambda^{2}\right)\right)}{W\left(f_{+}(\cdot, \lambda), \theta\left(\cdot, \lambda^{2}\right)\right)}\right] d \lambda, \\
& \rho_{2}(d \lambda):=-\frac{1}{\pi} \lambda \mathfrak{I}\left[\frac{W\left(f_{+}(\cdot, \lambda), \theta\left(\cdot, \lambda^{2}\right)\right)}{W\left(f_{+}(\cdot, \lambda), \phi\left(\cdot, \lambda^{2}\right)\right)}\right] d \lambda .
\end{aligned}
$$

By Proposition 4.4 (4.49) and (4.50) imply (4.42) and (4.43). It remains to prove (4.44). As in Section 3, the main difficulty is encountered when considering $0<\lambda \ll 1$. Indeed, it is not hard to show that if $\lambda$ is bounded away from $0, \lambda \gtrsim 1$, then the distorted Fourier basis $\theta\left(r, \lambda^{2}\right), \phi\left(r, \lambda^{2}\right)$ and measure $\Omega\left(d \lambda^{2}\right)$ in (4.49) are approximated to leading order by the free case (4.48). For the free case, (4.44) holds (for $\lambda \gtrsim 1$ ). Thus, (4.44) holds in the perturbed case for $\lambda \gtrsim 1$. We omit the details, and instead focus on establishing (4.44) in the case $0<\lambda \ll 1$. To establish (4.44) in the small $\lambda$ regime, we use the scattering theory summarized in Section 3 to derive asymptotic expansions for $\theta\left(r, \lambda^{2}\right), \phi\left(r, \lambda^{2}\right), \rho_{1}(d \lambda)$, and $\rho_{2}(d \lambda)$. The upcoming calculations will freely use the notation from Section 3.

We first consider the zero energy equation. Let $\theta_{0}(r), \phi_{0}(r)$ be the fundamental system for $H f=0$ such that

$$
\begin{array}{ll}
\theta_{0}(0)=1, & \phi_{0}(0)=0 \\
\theta_{0}^{\prime}(0)=0, & \phi_{0}^{\prime}(0)=1
\end{array}
$$

Then

$$
\begin{aligned}
& \phi_{0}(r)=a_{0} u_{0}^{+}(r)+a_{1} u_{1}^{+}(r), \\
& \theta_{0}(r)=b_{0} u_{0}^{+}(r)+b_{1} u_{1}^{+}(r),
\end{aligned}
$$

where, we recall that, the solutions $u_{j}^{+}(r)$ satisfy $H u_{j}^{+}(r)=0$ and

$$
\begin{aligned}
& u_{0}^{+}(r)=\frac{1}{3} r^{2}+O(r), \\
& u_{1}^{+}(r)=r^{-1}+O\left(r^{-2}\right),
\end{aligned}
$$

as $r \rightarrow \infty$ (see Lemma 3.3). Since $W\left(\theta_{0}, \phi_{0}\right)=1=W\left(u_{1}^{+}, u_{0}^{+}\right)$, we conclude that

$$
a_{0} b_{1}-a_{1} b_{0}=1 \text {. }
$$

Since $\phi_{0}$ and $\theta_{0}$ are odd and even respectively, the assumption that 0 is not a resonance implies the crucial condition that

$$
a_{0} \neq 0 \quad \text { and } \quad b_{0} \neq 0 .
$$

We now perturb in small $\lambda$. We claim that the smooth fundamental system $\theta\left(r, \lambda^{2}\right), \phi\left(r, \lambda^{2}\right)$ that satisfies (4.41) also satisfies

$$
\begin{aligned}
& \phi\left(r, \lambda^{2}\right)=\phi_{0}(r)+O\left(\lambda^{2}\langle r\rangle^{2} r^{2}\right), \\
& \theta\left(r, \lambda^{2}\right)=\theta_{0}(r)+O\left(\lambda^{2}\langle r\rangle^{2} r^{2}\right),
\end{aligned}
$$


for $0 \leq r \leq \lambda^{-1}$. The $O(\cdot)$ terms are real-valued and satisfy natural derivative bounds. Indeed, by variation of constants, we can write $\phi\left(r, \lambda^{2}\right)$ as a solution to

$$
\phi\left(r, \lambda^{2}\right)=\phi_{0}(r)+\lambda^{2} \int_{0}^{r}\left[u_{0}^{+}(r) u_{1}^{+}(\rho)-u_{0}^{+}(\rho) u_{1}^{+}(r)\right] \phi\left(\rho, \lambda^{2}\right) d \rho .
$$

If we define $\tilde{\phi}\left(r, \lambda^{2}\right)=\langle r\rangle^{-2} \phi\left(r, \lambda^{2}\right)$ and $K(r, \rho, \lambda)=\lambda^{2}\langle\rho\rangle^{2}\langle r\rangle^{-2}\left[u_{0}^{+}(r) u_{1}^{+}(\rho)-u_{0}^{+}(\rho) u_{1}^{+}(r)\right]$, then (4.57) takes the form of the Volterra equation

$$
\tilde{\phi}\left(r, \lambda^{2}\right)=\langle r\rangle^{-2} \phi_{0}(r)+\int_{0}^{r} K(r, \rho, \lambda) \tilde{\phi}\left(\rho, \lambda^{2}\right) d \rho .
$$

By (4.53), if $0<\rho<r$, then the kernel satisfies

$$
|K(r, \rho, \lambda)| \lesssim \lambda^{2}\langle\rho\rangle .
$$

Thus,

$$
\int_{0}^{\lambda^{-1}} \sup _{r>\rho}|K(r, \rho, \lambda)| d \rho \lesssim 1,
$$

which implies that the Volterra iterates for (4.58) converge on $\left[0, \lambda^{-1}\right]$ to a unique solution $\tilde{\phi}\left(r, \lambda^{2}\right)$ satisfying

$$
\tilde{\phi}\left(r, \lambda^{2}\right)=\langle r\rangle^{-2} \phi_{0}(r)+O\left(\lambda^{2} r^{2}\right) .
$$

This proves (4.56) for $\phi\left(r, \lambda^{2}\right)$. An identical argument proves (4.56) for $\theta\left(r, \lambda^{2}\right)$ as well. By Lemma 3.5]there exists a fundamental system $u_{0}^{+}(r, \lambda), u_{1}^{+}(r, \lambda)$ for $H f=\lambda^{2} f$ such that $W\left(u_{1}^{+}(\cdot, \lambda), u_{0}^{+}(\cdot, \lambda)\right)=1$ and for $j=0,1$

$$
u_{j}^{+}(r, \lambda)=u_{j}^{+}(r)\left(1+O\left(\langle r\rangle^{2} \lambda^{2}\right)\right), \quad r \in\left[r_{0}, \epsilon_{0} \lambda^{-1}\right],
$$

for some fixed $r_{0}, \epsilon_{0}>0$. Similar to (4.52), we can write

$$
\begin{aligned}
& \phi\left(r, \lambda^{2}\right)=a_{0}(\lambda) u_{0}^{+}(r, \lambda)+a_{1}(\lambda) u_{1}^{+}(r, \lambda), \\
& \theta\left(r, \lambda^{2}\right)=b_{0}(\lambda) u_{0}^{+}(r, \lambda)+b_{1}(\lambda) u_{1}^{+}(r, \lambda),
\end{aligned}
$$

with $a_{0}(\lambda) b_{1}(\lambda)-a_{1}(\lambda) b_{0}(\lambda)=1$. We claim that

$$
\begin{array}{ll}
a_{0}(\lambda)=a_{0}+O\left(\lambda^{2}\right), & a_{1}(\lambda)=a_{1}+O\left(\lambda^{2}\right), \\
b_{0}(\lambda)=b_{0}+O\left(\lambda^{2}\right), & b_{1}(\lambda)=b_{1}+O\left(\lambda^{2}\right),
\end{array}
$$

as $\lambda \rightarrow 0$ where $a_{0}, a_{1}, b_{0}$, and $b_{1}$ are as in (4.52). Indeed, using (4.56) and (4.59), we evaluate the Wronskian at $r=r_{0}$ and deduce that

$$
\begin{aligned}
a_{0}(\lambda) & =W\left(u_{1}^{+}(r, \lambda), \phi\left(r, \lambda^{2}\right)\right. \\
& =W\left(u_{1}^{+}(r)\left(1+O\left(\lambda^{2}\langle r\rangle^{2}\right), a_{0} u_{0}^{+}(r)+a_{1} u_{+}^{1}(r)+O\left(\lambda^{2}\langle r\rangle^{2} r^{2}\right)\right)\right. \\
& =a_{0}+O\left(\lambda^{2}\right) .
\end{aligned}
$$

The computation for $a_{1}, b_{0}$, and $b_{1}$ are similar so that (4.61) follows. We are now in a position to derive asymptotics for

$$
\begin{aligned}
& \omega_{1}(\lambda):=\frac{1}{\pi} \lambda \mathfrak{J}\left[\frac{W\left(f_{+}(\cdot, \lambda), \phi\left(\cdot, \lambda^{2}\right)\right)}{W\left(f_{+}(\cdot, \lambda), \theta\left(\cdot, \lambda^{2}\right)\right)}\right] \\
& \omega_{2}(\lambda):=-\frac{1}{\pi} \lambda \mathfrak{I}\left[\frac{W\left(f_{+}(\cdot, \lambda), \theta\left(\cdot, \lambda^{2}\right)\right)}{W\left(f_{+}(\cdot, \lambda), \phi\left(\cdot, \lambda^{2}\right)\right)}\right] .
\end{aligned}
$$

By Lemma 3.7, we have

$$
\begin{aligned}
& W\left(f_{+}(\cdot, \lambda), u_{1}^{+}(\cdot, \lambda)\right)=\alpha_{0}^{+}(\lambda)+i \alpha_{1}^{+}(\lambda) \\
& W\left(f_{+}(\cdot, \lambda), u_{0}^{+}(\cdot, \lambda)\right)=\beta_{0}^{+}(\lambda)+i \beta_{1}^{+}(\lambda)
\end{aligned}
$$


where

$$
\begin{aligned}
\alpha_{0}^{+}(\lambda)=\lambda^{2}\left(\alpha_{0}+O\left(\lambda^{\epsilon}\right)\right), & \alpha_{1}^{+}(\lambda)=O\left(\lambda^{2-2 \epsilon}\right), \\
\beta_{0}^{+}(\lambda)=O\left(\lambda^{-1+4 \epsilon}\right), & \beta_{1}^{+}(\lambda)=\lambda^{-1}\left(\beta_{0}+O\left(\lambda^{\epsilon}\right)\right),
\end{aligned}
$$

for all $0<\epsilon<\epsilon_{0}$. The constants $\alpha_{0}$ and $\beta_{0}$ in (4.63) are positive. From (4.60) and (4.62), we conclude that

$$
\begin{aligned}
& W\left(f_{+}(\cdot, \lambda), \phi\left(\cdot, \lambda^{2}\right)\right)=A_{0}(\lambda)+i A_{1}(\lambda), \\
& W\left(f_{+}(\cdot, \lambda), \theta\left(\cdot, \lambda^{2}\right)\right)=B_{0}(\lambda)+i B_{1}(\lambda),
\end{aligned}
$$

where

$$
\begin{aligned}
& A_{0}(\lambda)=a_{0}(\lambda) \beta_{0}^{+}(\lambda)+a_{1}(\lambda) \alpha_{0}^{+}(\lambda), \\
& A_{1}(\lambda)=a_{0}(\lambda) \beta_{1}^{+}(\lambda)+a_{1}(\lambda) \alpha_{1}^{+}(\lambda), \\
& B_{0}(\lambda)=b_{0}(\lambda) \beta_{0}^{+}(\lambda)+b_{1}(\lambda) \alpha_{0}^{+}(\lambda), \\
& B_{1}(\lambda)=b_{0}(\lambda) \beta_{1}^{+}(\lambda)+b_{1}(\lambda) \alpha_{1}^{+}(\lambda) .
\end{aligned}
$$

Then

$$
\mathfrak{J}\left[\frac{W\left(f_{+}(\cdot, \lambda), \phi\left(\cdot, \lambda^{2}\right)\right)}{W\left(f_{+}(\cdot, \lambda), \theta\left(\cdot, \lambda^{2}\right)\right)}\right]=\frac{A_{1} B_{0}-A_{0} B_{1}}{B_{0}^{2}+B_{1}^{2}} .
$$

By (4.63) and the condition that $a_{0}(\lambda) b_{1}(\lambda)-a_{1}(\lambda) b_{0}(\lambda)=1$, we conclude that

$$
\begin{aligned}
A_{1} B_{0}-A_{0} B_{1} & =\beta_{1}^{+}(\lambda) \alpha_{0}^{+}(\lambda)-\alpha_{1}^{+}(\lambda) \beta_{0}^{+}(\lambda) \\
& =\lambda\left(\alpha_{0} \beta_{0}+O\left(\lambda^{\epsilon}\right)\right) .
\end{aligned}
$$

By (4.63) and 4.61)

$$
B_{0}^{2}+B_{1}^{2}=\lambda^{-2}\left(b_{0}^{2} \beta_{0}^{2}+O\left(\lambda^{\epsilon}\right)\right) .
$$

Thus, (4.65), (4.66), and (4.67) yield

$$
\mathfrak{J}\left[\frac{W\left(f_{+}(\cdot, \lambda), \phi\left(\cdot, \lambda^{2}\right)\right)}{W\left(f_{+}(\cdot, \lambda), \theta\left(\cdot, \lambda^{2}\right)\right)}\right]=\lambda^{3} \frac{\alpha_{0} \beta_{0}+O\left(\lambda^{\epsilon}\right)}{b_{0}^{2} \beta_{0}^{2}+O\left(\lambda^{\epsilon}\right)}
$$

as $\lambda \rightarrow 0^{+}$. Similarly,

$$
-\mathfrak{J}\left[\frac{W\left(f_{+}(\cdot, \lambda), \theta\left(\cdot, \lambda^{2}\right)\right)}{W\left(f_{+}(\cdot, \lambda), \phi\left(\cdot, \lambda^{2}\right)\right)}\right]=\lambda^{3} \frac{\alpha_{0} \beta_{0}+O\left(\lambda^{\epsilon}\right)}{a_{0}^{2} \beta_{0}^{2}+O\left(\lambda^{\epsilon}\right)} .
$$

The crucial nonresonant condition (4.55) implies that (4.68) and (4.69) are both $O\left(\lambda^{3}\right)$. In summary, we have shown that the measures $\rho_{1}(d \lambda)=\omega_{1}(\lambda) d \lambda$ and $\rho_{2}(d \lambda)=\omega_{2}(\lambda) d \lambda$ in (4.50) have weights that satisfy

$$
\omega_{1}(\lambda)=O\left(\lambda^{4}\right), \quad \omega_{2}(\lambda)=O\left(\lambda^{4}\right) .
$$

We now prove (4.44) using the asymptotics from the previous paragraph. The expressions (4.56), (4.52), (4.53), and (4.70) imply that

$$
\left(\frac{1+\lambda^{2}\langle r\rangle^{2}}{\lambda^{2}\langle r\rangle^{2}}\right)\left[\left|\theta\left(r, \lambda^{2}\right)\right|^{2} \omega_{1}(\lambda)+\left|\phi\left(r, \lambda^{2}\right)\right|^{2} \omega_{2}(\lambda)\right] \lesssim 1, \quad r \in\left[0, \lambda^{-1}\right] .
$$

We now consider the case $r \geq \lambda^{-1}$. We first recall that

$$
W\left(f_{+}(\cdot, \lambda), \overline{f_{+}(\cdot, \lambda)}\right)=-2 i \lambda \neq 0,
$$

for $\lambda>0$. Thus, we can write

$$
\phi\left(r, \lambda^{2}\right)=c(\lambda) f_{+}(r, \lambda)+d(\lambda) \overline{f_{+}(r, \lambda)} .
$$

Since $\phi\left(r, \lambda^{2}\right)$ is real-valued, $d(\lambda)=\overline{c(\lambda)}$. Note that

$$
W\left(\phi\left(\cdot, \lambda^{2}\right), \overline{f_{+}(\cdot, \lambda)}\right)=-2 i \lambda c(\lambda),
$$


so that by (4.62)-(4.64), we conclude that

$$
c(\lambda)=\frac{1}{2 i \lambda} W\left(f_{+}(\cdot, \lambda), \phi\left(\cdot, \lambda^{2}\right)\right)=O_{\mathbb{C}}\left(\lambda^{-2}\right) .
$$

By Lemma 3.6 we have

$$
f_{+}(r, \lambda)=e^{i r \lambda}\left(1+O\left(\lambda^{-1}\langle r\rangle^{-1}\right)\right), \quad r \geq \lambda^{-1} .
$$

From (4.70)-4.74), we conclude that

$$
\left(\frac{1+\lambda^{2}\langle r\rangle^{2}}{\lambda^{2}\langle r\rangle^{2}}\right)\left|\phi\left(r, \lambda^{2}\right)\right|^{2} \omega_{2}(\lambda) \lesssim 1, \quad r \geq \lambda^{-1} .
$$

By the exact same arguments,

$$
\left(\frac{1+\lambda^{2}\langle r\rangle^{2}}{\lambda^{2}\langle r\rangle^{2}}\right)\left|\theta\left(r, \lambda^{2}\right)\right|^{2} \omega_{1}(\lambda) \lesssim 1, \quad r \geq \lambda^{-1} .
$$

In summary, we have shown that for $0<\lambda \ll 1$,

$$
\left(\frac{1+\lambda^{2}\langle r\rangle^{2}}{\lambda^{2}\langle r\rangle^{2}}\right)\left[\left|\theta\left(r, \lambda^{2}\right)\right|^{2} \omega_{1}(\lambda)+\left|\phi\left(r, \lambda^{2}\right)\right|^{2} \omega_{2}(\lambda)\right] \lesssim 1, \quad r \in \mathbb{R} .
$$

This proves (4.44) and concludes the proof of Proposition 4.3 .

\section{Small Data Theory and Concentration-Compactness}

In this section we use the tools developed in the previous sections to initiate the study of the nonlinear evolution introduced in the previous section:

$$
\begin{aligned}
& \partial_{t}^{2} u-\Delta_{g} u+V(r) u=N(r, u), \quad(t, r) \in \mathbb{R} \times \mathbb{R}, \\
& \vec{u}(0)=\left(u_{0}, u_{1}\right) \in \mathcal{H},
\end{aligned}
$$

where $\mathcal{H}:=\mathcal{H}\left(\mathbb{R} ;\left(r^{2}+1\right)^{2} d r\right),-\Delta_{g}$ is the (radial) Laplace operator on the $5 d$ wormhole $\mathcal{M}^{5}$, and $V(r)$ and $N(r, u)$ are given in (4.5) and (4.6). In particular, we begin our proof of Theorem 4.1, i.e. every solution to (5.1) is global and scatters to free waves on $\mathcal{M}^{5}$.

5.1. Small Data Theory. As summarized in the introduction, the proof of Theorem 4.1 (which we have shown in Section 4 is equivalent to Theorem (1.1)) uses the powerful concentration-compactness/rigidity methodology introduced by Kenig and Merle in their study of energy-critical dispersive equations [12] [13]. The methodology is split up into three main steps and proceeds by contradiction. In the first step, we establish small data global well-posedness and scattering for (4.4). In particular, we establish Theorem 4.1 for small data $\left(u_{0}, u_{1}\right)$. In the second step, the first step and a concentration-compactness argument shows that the failure of Theorem 4.1 implies that that there exists a nonzero 'critical element' $u_{*}$; a minimal non-scattering global solution to (4.4). The minimality of $u_{*}$ imposes the following compactness property on $u_{*}$ : the trajectory

$$
K=\left\{\vec{u}_{*}(t): t \in \mathbb{R}\right\}
$$

is precompact in $\mathcal{H}$. In the third and final step, we establish the following rigidity theorem: every solution $u$ with $\{\vec{u}(t): t \in \mathbb{R}\}$ precompact in $\mathcal{H}$ must identically 0 . This contradicts the second step which implies that Theorem 4.1 holds. In this section we complete the first two steps in the program: small data theory and concentration-compactness. These steps follow from, by now, standard arguments using the Strichartz estimates for $\partial_{t}^{2}-\Delta_{g}+V$ established in Section 4 .

We first establish a global well-posedness and small data theory for (4.4). This follows from a contraction mapping argument using Strichartz estimates established in Proposition 4.2 for the inhomogeneous wave equation with potential

$$
\begin{aligned}
& \partial_{t}^{2} u-\Delta_{g} u+V(r) u=h(t, r), \quad(t, r) \in \mathbb{R} \times \mathbb{R}, \\
& \vec{u}(0)=\left(u_{0}, u_{1}\right) \in \mathcal{H} .
\end{aligned}
$$


Here, as in the previous section, the potential $V$ is given by

$$
V(r)=\langle r\rangle^{-4}+2\langle r\rangle^{-2}(\cos 2 Q-1),
$$

where $Q$ is the unique harmonic map of degree $n$. To see that $V$ satisfies the hypotheses in Proposition 4.2 , we note that by Proposition 2.1, we only need to verify the spectral assumptions are satisfied. This was shown in [3], and we recall the argument. We have the relation

$$
\langle r\rangle^{2}\left(-\Delta_{g}+V\right)\langle r\rangle^{-2}=H,
$$

where $H$ is the Schrödinger operator on $L^{2}(\mathbb{R})$ given by

$$
H=-\frac{d^{2}}{d r^{2}}+\frac{2}{r^{2}+1}+V(r)
$$

We need to check that $H$ has no point spectrum and that 0 is not a resonance for $H$. First, we note that the decay of the potential $\frac{2}{r^{2}+1}+V(r)$ implies that $\sigma_{a c}(H)=[0, \infty)$ and there are no embedded eigenvalues. If $Q \equiv 0$ (the $n=0$ case), the fact that $H$ has no eigenvalues in $(-\infty, 0]$ follows from the fact that the potential term $2\langle r\rangle^{-2}+V(r)$ is nonnegative. For the case $n \in \mathbb{N}$, multiply the equation

$$
\partial_{r}^{2} Q+\frac{2 r}{r^{2}+1} \partial_{r} Q-\frac{\sin 2 Q}{r^{2}+1}=0
$$

by $r^{2}+1$ and differentiate to conclude that

$$
\tilde{H}\left(\langle r\rangle^{2} Q^{\prime}(r)\right)=0,
$$

where $\tilde{H}=H-\langle r\rangle^{4}$. By Proposition 2.1 the harmonic map $Q$ is strictly increasing on $\mathbb{R}$ so that $\left.\langle r\rangle^{2} Q^{\prime}(r)\right\rangle 0$ for all $r \in \mathbb{R}$. By Sturm oscillation theory we conclude that $\tilde{H}$ has no negative eigenvalues and that $\sigma(\tilde{H})=[0, \infty)$. In particular, we have for all $h \in C_{0}^{\infty}(\mathbb{R})$

$$
(H h, h)_{L^{2}(\mathbb{R})}=(\tilde{H} h, h)_{L^{2}(\mathbb{R})}+\int|h|^{2}\langle r\rangle^{-4} d r \geq \int|h|^{2}\langle r\rangle^{-4} d r .
$$

By a variational principle, the previous implies that $H$ has no eigenvalues in $(-\infty, 0]$, and thus, $H$ has no point spectrum. We now check that 0 is not a resonance of $H$. The asymptotics of the potential $2\langle r\rangle^{-2}+V(r)$ imply that 0 is a resonance if and only if 0 is an eigenvalue (see Lemma 3.3 and Definition 3.4). Thus, 0 is not a resonance of $H$. We conclude that $V$ satisfies the hypotheses of Proposition 4.2.

For $I \subseteq \mathbb{R}$, we denote the following spacetime norms

$$
\|u\|_{S(I)}:=\|u\|_{L_{t}^{3} L_{x}^{6}(I)}, \quad\|u\|_{W(I)}:=\|u\|_{L_{t}^{3} \dot{W}_{x}^{1 / 2 L^{3}(I)}} \quad\|h\|_{N(I)}:=\|F\|_{L_{t}^{1} L_{x}^{2}(I)+L_{t}^{3 / 2} \dot{W}_{x}^{1 / 2,3 / 2}(I)} .
$$

By the previous discussion and Proposition 4.2. a solution $u$ to (5.2) satisfies the estimate

$$
\|u\|_{W(I)} \lesssim\|\vec{u}(0)\|_{\mathcal{H}}+\|h\|_{N(I)} .
$$

We claim that if $f \in C_{0}^{\infty}\left(\mathcal{M}^{5}\right)$ is radial, then

$$
\|f\|_{L_{x}^{6}} \lesssim\left\|\left.\nabla\right|^{1 / 2} f\right\|_{L_{x}^{3}} .
$$

Indeed, by the fundamental theorem of calculus, we have

$$
|f(r)| \lesssim\langle r\rangle^{-2 / 3}\left(\int\left|f^{\prime}(r)\right|^{3}\left(r^{2}+1\right) d r\right)^{1 / 3}=\langle r\rangle^{-2 / 3}\|\nabla f\|_{L_{x}^{3}} .
$$

Thus, $\|f\|_{L_{x}^{\infty}} \lesssim\|\nabla f\|_{L_{x}^{3}}$. Interpolating this estimate with the trivial embedding $L_{x}^{3} \hookrightarrow L_{x}^{3}$ yields the desired bound $\|f\|_{L_{x}^{6}} \lesssim\left\||\nabla|^{1 / 2} f\right\|_{L_{x}^{3}}$. Thus, we have that the 'scattering norm' $\|\cdot\|_{S(I)}$ is weaker than the norm $\|\cdot\|_{W(I)}$. This fact and (5.5) imply that a solution to (5.2) satisfies the Strichartz estimate

$$
\|u\|_{S(\mathbb{R})}+\|u\|_{W(\mathbb{R})} \lesssim\|\vec{u}(0)\|_{\mathcal{H}}+\|h\|_{N(\mathbb{R})} .
$$

We now use (5.6) and standard contraction mapping arguments to establish the following global wellposedness and small data theory. We remark here that it will be important in later applications to use the weaker norm $\|\cdot\|_{S(I)}$ along with the norm $\|\cdot\|_{W(\mathbb{R})}$ when establishing the small data scattering. 
Proposition 5.1. For every $\left(u_{0}, u_{1}\right) \in \mathcal{H}$, there exists a unique global solution $u$ to (5.1) such that $\vec{u}(t) \in C(\mathbb{R} ; \mathcal{H}) \cap$ $L^{\infty}(\mathbb{R} ; \mathcal{H})$. A solution $u$ scatters to a free wave on $\mathcal{M}^{5}$ as $t \rightarrow \infty$ if and only if

$$
\|u\|_{S(\mathbb{R})}<\infty \text {. }
$$

Here, scattering to a free wave on $\mathcal{M}^{5}$ as $t \rightarrow \infty$ means that there exists a solution $v_{L}$ to (5.2) with $V \equiv h \equiv 0$ such that

$$
\lim _{t \rightarrow \infty}\left\|\vec{u}(t)-\vec{v}_{L}(t)\right\|_{\mathcal{H}}=0 .
$$

A similar characterization of $u$ scattering to a free wave on $\mathcal{M}^{5}$ as $t \rightarrow-\infty$ also holds. Moreover, there exists $\delta>0$ such that if $\|\vec{u}(0)\|_{\mathcal{H}}<\delta$, then

$$
\|\vec{u}\|_{L_{t}^{\infty} \mathcal{H}}+\|u\|_{S(\mathbb{R})}+\|u\|_{W(\mathbb{R})} \lesssim\|\vec{u}(0)\|_{\mathcal{H}}<\delta .
$$

Proof. We first show that for every $\left(u_{0}, u_{1}\right) \in \mathcal{H}$, there exists a unique global solution $\vec{u}(t) \in C(\mathbb{R} ; \mathcal{H}) \cap L^{\infty}(\mathbb{R} ; \mathcal{H})$ to (5.1) with $\vec{u}(0)=\left(u_{0}, u_{1}\right)$. Denote the propagator for the free wave equation on $\mathcal{M}^{5}$ by $S(t)$, i.e. $S(t)\left(u_{0}, u_{1}\right)$ solves (5.2) with $V \equiv h \equiv 0$. Denote the propagator for the free wave equation on $\mathcal{M}^{5}$ with potential $V$ by $S_{V}(t)$, i.e. $S_{V}(t)\left(u_{0}, u_{1}\right)$ solves (5.2) with $h \equiv 0$. Let

$$
\mathcal{E}_{V}(f, g):=\frac{1}{2} \int\left(|g|^{2}+\left|\partial_{r} f\right|^{2}+V|f|^{2} d r\right)\left(r^{2}+1\right)^{2} d r
$$

denote the conserved energy associated to $S_{V}$. Using the coercivity bound (5.4) it is not hard to conclude that

$$
\left\|\partial_{r} f\right\|_{L^{2}\left(\mathbb{R} ;\left(r^{2}+1\right)^{2}\right)} \simeq\left\|\sqrt{-\Delta_{g}+V} f\right\|_{L^{2}\left(\mathbb{R} ;\left(r^{2}+1\right)^{2}\right)}
$$

for all radial $f$ so that

$$
\|(f, g)\|_{\mathcal{H}}^{2} \simeq \mathcal{E}_{V}(f, g)
$$

for all radial $f, g$. Indeed, by the decay of $V$ and the Strauss estimate (4.13) we have

$$
\begin{aligned}
\left\|\sqrt{-\Delta_{g}+V} f\right\|_{L^{2}\left(\mathbb{R} ;\left(r^{2}+1\right)^{2}\right)}^{2} & =\int\left(\left(-\Delta_{g} f\right) f+V|f|^{2}\right)\left(r^{2}+1\right)^{2} d r \\
& =\left\|\partial_{r} f\right\|_{L^{2}\left(\mathbb{R} ;\left(r^{2}+1\right)^{2} d r\right)}^{2}+\int V|f|^{2}\left(r^{2}+1\right)^{2} d r \\
& \lesssim\left\|\partial_{r} f\right\|_{L^{2}\left(\mathbb{R} ;\left(r^{2}+1\right)^{2} d r\right)}^{2} .
\end{aligned}
$$

We now note that by the second equality above and the decay of $V$ we have

$$
\left\|\partial_{r} f\right\|_{L^{2}\left(\mathbb{R} ;\left(r^{2}+1\right)^{2} d r\right)}^{2} \lesssim\left\|\sqrt{-\Delta_{g}+V} f\right\|_{L^{2}\left(\mathbb{R} ;\left(r^{2}+1\right)^{2}\right)}^{2}+\int|f|^{2} d r .
$$

By (5.3) and (5.4) (applied to $\left.h=\left(r^{2}+1\right) f\right)$ we see that

$$
\int|f|^{2} d r \lesssim\left\|\sqrt{-\Delta_{g}+V} f\right\|_{L^{2}\left(\mathbb{R} ;\left(r^{2}+1\right)^{2}\right)}^{2}
$$

whence

$$
\left\|\partial_{r} f\right\|_{L^{2}\left(\mathbb{R} ;\left(r^{2}+1\right)^{2} d r\right)}^{2} \lesssim\left\|\sqrt{-\Delta_{g}+V} f\right\|_{L^{2}\left(\mathbb{R} ;\left(r^{2}+1\right)^{2}\right)}^{2} .
$$

This proves (5.9).

We write the nonlinear equation (5.1) in Duhamel form as

$$
u(t)=S_{V}(t)\left(u_{0}, u_{1}\right)+\int_{0}^{t} S_{V}(t-s)(0, F(\cdot, u(s))+G(\cdot, u(s))) d s
$$


Using a simple energy estimate, (4.8), (4.9), (4.10), (4.13), and (5.10), we obtain the following a-priori estimate for a solution $\vec{u}(t) \in C([0, T] ; \mathcal{H})$ to $(5.1)$ : for $t \in[0, T]$

$$
\begin{aligned}
\|\vec{u}(t)\|_{\mathcal{H}} & \lesssim\|\vec{u}(0)\|_{\mathcal{H}}+\int_{0}^{T} \| F(\cdot, u(s))+G\left(\cdot, u(s) \|_{L^{2}} d s\right. \\
& \lesssim\|\vec{u}(0)\|_{\mathcal{H}}+T\left(\|\vec{u}\|_{L_{t}^{\infty}([0, T] ; \mathcal{H})}^{2}+\|\vec{u}\|_{L_{t}^{\infty}([0, T] ; \mathcal{H})}^{3}\right) .
\end{aligned}
$$

By a contraction mapping argument based on (5.11) and the conservation of energy for (4.1), we conclude that there exists a unique global solution $\vec{u}(t) \in C(\mathbb{R} ; \mathcal{H}) \cap L^{\infty}(\mathbb{R} ; \mathcal{H})$ to $(5.1)$.

We now prove the scattering criterion and small data scattering. Note that every solution $\vec{u}(t) \in C(\mathbb{R} ; \mathcal{H})$ to (5.1) satisfies $\|u\|_{S(I)}+\|u\|_{W(I)}<\infty$ for all $I \Subset \mathbb{R}$. Indeed, by (4.13) we have $\|u\|_{L_{x}^{6}} \lesssim\|\nabla u\|_{L_{x}^{2}}$ whence by interpolation we have $\|u\|_{\dot{W}_{x}^{1 / 2,3}} \lesssim\|\nabla u\|_{L_{x}^{2}}$. We first prove the small data scattering estimate (5.7) as this will also illustrate the validity of the scattering criterion. Let $u$ be a solution to (5.1) and let $I \subset \mathbb{R}$. We first note that by the Leibniz rule for Sobolev spaces (see [4] for asymptotically conic manifolds), we have

$$
\left\|\left(\langle r\rangle^{-1} \sin 2 Q\right) u^{2}\right\|_{\dot{W}_{x}^{1 / 2,3 / 2}} \lesssim\left\|\langle r\rangle^{-1} \sin 2 Q\right\|_{\dot{W}_{x}^{1 / 2,3}}\left\|u^{2}\right\|_{L_{x}^{3}}+\left\|\langle r\rangle^{-1} \sin 2 Q\right\|_{L_{x}^{6}}\left\|u^{2}\right\|_{W_{x}^{1 / 2,2}} \lesssim\|u\|_{L_{x}^{6}}^{2}+\|u\|_{L_{x}^{6}}\|u\|_{W_{x}^{1 / 2,3}}
$$

whence by Hölder's inequality in time we have

$$
\left\|\left(\langle r\rangle^{-1} \sin 2 Q\right) u^{2}\right\|_{L_{t}^{3 / 2} \dot{W}_{x}^{1 / 2,3 / 2}(I)} \lesssim\|u\|_{S(I)}^{2}+\|u\|_{S(I)}\|u\|_{W(I)} .
$$

Then by (4.8), (4.9), (4.10), the Strichartz estimate (5.6), and (5.12) we have

$$
\begin{aligned}
\|u\|_{S(I)}+\|u\|_{W(I)} & \lesssim\|\vec{u}(0)\|_{\mathcal{H}}+\|N(\cdot, u)\|_{N(I)} \\
& \lesssim\|\vec{u}(0)\|_{\mathcal{H}}+\|F(\cdot, u)\|_{N(I)}+\|G(\cdot, u)\|_{N(I)} \\
& \lesssim\|\vec{u}(0)\|_{\mathcal{H}}++\left\|\left(\langle r\rangle^{-1} \sin 2 Q\right) u^{2}\right\|_{L_{t}^{3 / 2} \dot{W}_{x}^{1 / 2,3 / 2}(I)}+\left\|\langle r\rangle^{-1} u^{4}\right\|_{L_{t}^{1} L_{x}^{2}(I)}+\left\|\left.u\right|^{3}\right\|_{L_{t}^{1} L_{x}^{2}(I)} \\
& \lesssim\|\vec{u}(0)\|_{\mathcal{H}}+\|u\|_{S(I)}\|u\|_{W(I)}+\|u\|_{S(I)}^{2}+\|\vec{u}\|_{L_{t}^{\infty} \mathcal{H}}\|u\|_{S(I)}^{3}+\|u\|_{S(I)}^{3} .
\end{aligned}
$$

By a standard continuity argument, there exists $\delta>0$ such that if $\|\vec{u}(0)\|_{\mathcal{H}}<\delta$ then $\|\vec{u}\|_{L_{t}^{\infty} \mathcal{H}}+\|u\|_{S(\mathbb{R})}+\|u\|_{W(\mathbb{R})} \lesssim$ $\|\vec{u}(0)\|_{\mathcal{H}}$ as desired. A simple variant of the above argument also shows that if $\|u\|_{S(0, \infty)}<\infty$, then

$$
w_{L}(0)=\vec{u}(0)+\int_{0}^{\infty} S_{V}(-s)(0, N(\cdot, u(s))) d s
$$

converges in $\mathcal{H}$. Thus, by Duhamel we conclude that

$$
\vec{u}(t)=\vec{S}_{V}(t) w_{L}(0)+o_{\mathcal{H}}(1),
$$

as $t \rightarrow \infty$. To extract a free wave $v_{L}(t)=S(t) \vec{v}_{L}(0)$ from the perturbed wave $w_{L}(t)=S_{V}(t) \vec{w}_{L}(0)$, we write, via Duhamel,

$$
\begin{aligned}
w_{L}(t) & =S(t) \vec{w}_{L}(0)+\int_{0}^{t} S(t-s)\left(0, V w_{L}(s)\right) d s \\
& =S(t)\left[\vec{w}_{L}(0)+\int_{0}^{t} S(-s)\left(0, V w_{L}(s)\right) d s\right] .
\end{aligned}
$$

We then take

$$
\vec{v}_{L}(0)=\vec{w}_{L}(0)+\int_{0}^{\infty} S(-s)\left(0, V w_{L}(s)\right) d s
$$

which converges in $\mathcal{H}$ by (4.23) with $X=L_{t}^{\infty} \mathcal{H}$. Then $\vec{w}_{L}(t)=\vec{v}_{L}(t)+o_{\mathcal{H}}(1)$ as $t \rightarrow \infty$. This along with (5.13) allow us to conclude that if $\|u\|_{S(0, \infty)}<\infty$, then $u$ scatters to a free wave on $\mathcal{M}^{5}$ as $t \rightarrow \infty$. The fact that the finiteness of $\|u\|_{S(0, \infty)}$ is necessary if $u$ scatters as $t \rightarrow \infty$ follows from similar arguments using the fact that $\left\|v_{L}\right\|_{S(0, \infty)}<\infty$ holds for any free wave $v_{L}$ on $\mathcal{M}^{5}$. This concludes the proof.

A tool that will be essential in establishing the second step of the concentration-compactness/rigidity theorem method is the following long-time perturbation theory for (5.1). 
Proposition 5.2 (Long-time perturbation theory). Let $A>0$. Then there exists $\epsilon_{0}=\epsilon_{0}(A)>0$ and $C=C(A)>0$ such that the following holds. Let $0<\epsilon<\epsilon_{0},\left(u_{0}, u_{1}\right) \in \mathcal{H}$, and $I \subseteq \mathbb{R}$ with $0 \in I$. Assume that $\vec{U}(t) \in C(I ; \mathcal{H})$ satisfies on I

$$
\partial_{t}^{2} U-\Delta_{g} U+V U=N(\cdot, U)+e,
$$

such that

$$
\begin{array}{r}
\sup _{t \in I}\|\vec{U}(t)\|_{\mathcal{H}}+\|U\|_{S(I)} \leq A, \\
\left\|\vec{U}(0)-\left(u_{0}, u_{1}\right)\right\|_{\mathcal{H}}+\|e\|_{N(I)} \leq \epsilon .
\end{array}
$$

Then the unique global solution $u$ to (5.1) with initial data $\vec{u}(0)=\left(u_{0}, u_{1}\right)$ satisfies

$$
\sup _{t \in I}\|\vec{u}(t)-\vec{U}(t)\|_{\mathcal{H}}+\|u-U\|_{S(I)} \leq C(A) \epsilon .
$$

Proof. We establish the estimate (5.17) with $I_{+}:=\operatorname{I\cap }[0, \infty)$ in place of $I$. Establishing (5.17) with $I_{-}:=\operatorname{I\cap }(-\infty, 0]$ in place of $I$ is similar, and these two estimates yield (5.17). We first make some preliminary observations. The bounds (5.15) and (5.16) along with conservation of energy imply that

$$
\|\vec{u}(t)\|_{\mathcal{H}} \leq C_{0}(A)
$$

Also, by interpolation and $(5.15),\|U\|_{W(f)}<\infty$ for all $J \Subset I$. We claim that

$$
\|U\|_{W(I)} \leq C_{1}(A) \text {. }
$$

To see this, let $\eta>0$ to be chosen later, and partition $I_{+}$into subintervals $I_{+}=\cup_{j=1}^{J_{0}(A)} I_{j}$ such that $\forall j,\|U\|_{S\left(I_{j}\right)}<\eta$. Then via (5.14) and Duhamel, we have on $I_{j}:=\left[t_{j}, t_{j+1}\right]$

$$
U(t)=S_{V}\left(t-t_{j}\right) \vec{U}\left(t_{j}\right)+\int_{t_{j}}^{t} S_{V}(t-s)(0, N(\cdot, U(s))+e) d s .
$$

By arguing as in the proof of Proposition 5.1 and Strichartz estimates, we have

$$
\begin{aligned}
\|U\|_{W\left(I_{j}\right)} & \leq C\left\|\vec{U}\left(t_{j}\right)\right\|_{\mathcal{H}}+C\|N(\cdot, U)\|_{N\left(I_{j}\right)}+C\|e\|_{N\left(I_{j}\right)} \\
& \leq C A+C\|U\|_{W\left(I_{j}\right)}\|U\|_{S\left(I_{j}\right)}+C\|U\|_{S\left(I_{j}\right)}^{2}+C\|\vec{U}\|_{L_{t}^{\infty} \mathcal{H}\left(I_{j}\right)}\|U\|_{S\left(I_{j}\right)}^{3}+C\|U\|_{S\left(I_{j}\right)}^{3}+C \epsilon \\
& \leq C \eta\|U\|_{W\left(I_{j}\right)}+C \epsilon+C \cdot(A+1)^{4} .
\end{aligned}
$$

If we choose $\eta=(2 C)^{-1}$, then we obtain (5.19).

We now establish (5.17). Define $w=u-U$. Then $w$ solves on $I$

$$
\begin{aligned}
& \partial_{t}^{2} w-\Delta_{g} w+V w=N(\cdot, U+w)-N(\cdot, U)-e, \\
& \vec{w}(0)=\left(u_{0}, u_{1}\right)-\vec{U}(0) .
\end{aligned}
$$

By (5.15) and (5.18), w satisfies

$$
\sup _{t \in I}\|\vec{w}(t)\|_{\mathcal{H}} \leq A+C_{0}(A)
$$

Let $\eta>0$ to be chosen later. Partition $I_{+}$into subintervals $I=\cup_{j=1}^{J_{1}(A)} I_{j}$ such that

$$
\forall j, \quad\|U\|_{S\left(I_{j}\right)}+\|U\|_{W\left(I_{j}\right)} \leq \eta
$$

On $I_{j}:=\left[t_{j}, t_{j+1}\right]$, we have via (5.20) and Duhamel

$$
w(t)=S_{V}\left(t-t_{j}\right) \vec{w}\left(t_{j}\right)+\int_{t_{j}}^{t} S_{V}(t-s)(0, N(\cdot, U(s)+w(s))-N(\cdot, U(s))-e) d s .
$$


By arguing as in the proof of Proposition 5.1 and Strichartz estimates, we have

$$
\begin{aligned}
\|w\|_{S\left(I_{j}\right)}+\|w\|_{W\left(I_{j}\right)} & \leq\left\|S_{V}\left(t-t_{j}\right) \vec{w}\left(t_{j}\right)\right\|_{S(\mathbb{R})}+\left\|S_{V}\left(t-t_{j}\right) \vec{w}\left(t_{j}\right)\right\|_{W(\mathbb{R})}+C\|e\|_{N\left(I_{j}\right)} \\
& +C\|N(\cdot, U+w)-N(\cdot, U)\|_{N\left(I_{j}\right)} \\
& \leq\left\|S_{V}\left(t-t_{j}\right) \vec{w}\left(t_{j}\right)\right\|_{S(\mathbb{R})}+\left\|S_{V}\left(t-t_{j}\right) \vec{w}\left(t_{j}\right)\right\|_{W(\mathbb{R})}+C\|e\|_{N\left(I_{j}\right)} \\
& +C\left[\|w\|_{W\left(I_{j}\right)}\|U\|_{S\left(I_{j}\right)}+\|w\|_{S\left(I_{j}\right)}\|U\|_{W\left(I_{j}\right)}+\|w\|_{S\left(I_{j}\right)}\|w\|_{W\left(I_{j}\right)}+\|w\|_{S\left(I_{j}\right)}^{2}\right. \\
& +\|w\|_{S\left(I_{j}\right)}\|U\|_{S\left(I_{j}\right)}+\|w\|_{S\left(I_{j}\right)}\|U\|_{S\left(I_{j}\right)}^{2}\left(\|U\|_{L_{t}^{\infty} \mathcal{H}\left(I_{j}\right)}+1\right)+\|w\|_{S\left(I_{j}\right)}^{2}\|U\|_{S\left(I_{j}\right)}\left(\|U\|_{L_{t}^{\infty} \mathcal{H}\left(I_{j}\right)}+1\right) \\
& \left.+\|w\|_{S\left(I_{j}\right)}^{3}\left(\|U\|_{L_{t}^{\infty} \mathcal{H}}+\|w\|_{L_{t}^{\infty} \mathcal{H}\left(I_{j}\right)}+1\right)\right] \\
& \leq\left\|S_{V}\left(t-t_{j}\right) \vec{w}\left(t_{j}\right)\right\|_{S(\mathbb{R})}+\left\|S_{V}\left(t-t_{j}\right) \vec{w}\left(t_{j}\right)\right\|_{W(\mathbb{R})}+C \epsilon \\
& +\left(\eta+\eta^{2}\right)(A+1) C\left[\|w\|_{S\left(I_{j}\right)}+\|w\|_{W\left(I_{j}\right)}\right] \\
& +C_{2}(A)\left[\left(\|w\|_{S\left(I_{j}\right)}+\|w\|_{W\left(I_{j}\right)}\right)^{2}+\left(\|w\|_{S\left(I_{j}\right)}+\|w\|_{W\left(I_{j}\right)}\right)^{3}\right] .
\end{aligned}
$$

Here $C_{2}=C_{2}(A)$ is a constant which depends only $A$. Define

$$
\gamma_{j}:=\left\|S_{V}\left(t-t_{j}\right) \vec{w}\left(t_{j}\right)\right\|_{S(\mathbb{R})}+\left\|S_{V}\left(t-t_{j}\right) \vec{w}\left(t_{j}\right)\right\|_{w(\mathbb{R})}+C \epsilon .
$$

If we fix $\eta$ so small so that $\eta+\eta^{2}<(2(A+1) C)^{-1}$, then we obtain

$$
\|w\|_{S\left(I_{j}\right)}+\|w\|_{W\left(I_{j}\right)} \leq 2 \gamma_{j}+2 C_{1}(A)\left[\left(\|w\|_{S\left(I_{j}\right)}+\|w\|_{W\left(I_{j}\right)}\right)^{2}+\left(\|w\|_{S\left(I_{j}\right)}+\|w\|_{W\left(I_{j}\right)}\right)^{3}\right] .
$$

In particular, by a standard continuity argument there exists $\delta_{0}=\delta_{0}\left(C_{1}(A)\right)$ such that if $\gamma_{j}<\delta_{0}$, then

$$
\begin{aligned}
\|w\|_{S\left(I_{j}\right)}+\|w\|_{W\left(I_{j}\right)} & \leq 4 \gamma_{j}, \\
2 C_{2}(A)\left[\left(\|w\|_{S\left(I_{j}\right)}+\|w\|_{W\left(I_{j}\right)}\right)^{2}+\left(\|w\|_{S\left(I_{j}\right)}+\|w\|_{W\left(I_{j}\right)}\right)^{3}\right] & \leq 4 \gamma_{j} .
\end{aligned}
$$

We now iterate, and insert $t_{j+1}$ into (5.23). Applying $S_{V}\left(t-t_{j+1}\right)$ to both sides, we obtain

$$
S\left(t-t_{j+1}\right) \vec{w}\left(t_{j+1}\right)=S\left(t-t_{j}\right) \vec{w}\left(t_{j}\right)+\int_{t_{j}}^{t_{j+1}} S(t-s)(0, N(\cdot, U(s)+w(s))-N(\cdot, U(s))-e) d s .
$$

By (5.25) and (5.26) and the previous arguments, we deduce that

$$
\gamma_{j+1} \leq 10 \gamma_{j}
$$

provided that $\gamma_{j}<\delta_{0}$. By Strichartz estimates and (5.16), we have for some absolute constant $C_{3}$

$$
\gamma_{1}:=\left\|S_{V}(t) \vec{w}(0)\right\|_{S(\mathbb{R})}+\left\|S_{V}(t) \vec{w}(0)\right\|_{S(\mathbb{R})}+C \epsilon \leq C_{3} \epsilon<C_{3} \epsilon_{0} .
$$

Iterating, we have that $\gamma_{j+1} \leq 10^{j} C_{3} \epsilon$ as long as $\gamma_{j}<\delta_{0}$. If we choose $\epsilon_{0}=\epsilon_{0}(A)$ so small so that $10^{J} C_{3} \epsilon_{0}<\delta_{0}$, then the condition $\gamma_{j}<\delta_{0}$ is always satisfied. This along with (5.25) imply that

$$
\|w\|_{S\left(I_{+}\right)}+\|w\|_{W\left(I_{+}\right)} \leq C(A) \epsilon
$$

as desired. The estimate for $\|w\|_{L_{t}^{\infty} \mathcal{H}\left(I_{+}\right)}$follows a posteriori from (5.20), (5.15), (5.16), the estimate for $\|w\|_{S\left(I_{+}\right)}+\|w\|_{W\left(I_{+}\right)}$, and Strichartz estimates. This completes the proof.

5.2. Concentration-compactness. In this, the second step of the concentration-compactness methodology, we show that if our main result Theorem 4.1 (or equivalently Theorem 1.1) fails, then there exists a nonzero 'critical element.' More precisely, we prove the following.

Proposition 5.3. Suppose that Theorem 4.1 fails. Then there exists a nonzero global solution $u_{*}$ to (5.1) such that the set

$$
K=\left\{\vec{u}_{*}(t): t \in \mathbb{R}\right\}
$$

is precompact in $\mathcal{H}$.

Essential tools for proving Proposition 5.3 are the following linear and nonlinear profile decompositions. 
Lemma 5.4 (Linear Profile Decomposition). Let $\left\{\left(u_{0, n}, u_{1, n}\right)\right\}_{n}$ be a bounded sequence in $\mathcal{H}$. Then after extraction of subsequences and relabeling, there exist a sequence of solutions $\left\{U_{L}^{j}\right\}_{j \geq 1}$ to (5.2) with $h \equiv 0$ which are bounded in $\mathcal{H}$ and a sequence of times $\left\{t_{j, n}\right\}_{n}$ for $j \geq 1$ that satisfy the orthogonality condition

$$
\forall j \neq k, \quad \lim _{n \rightarrow \infty}\left|t_{j, n}-t_{k, n}\right|=\infty,
$$

such that for all $\mathrm{J} \geq 1$,

$$
\left(u_{0, n}, u_{1, n}\right)=\sum_{j=1}^{J} \vec{u}_{L}^{j}\left(-t_{j, n}\right)+\left(w_{0, n^{\prime}}^{J}, w_{1, n}^{J}\right),
$$

where the error $w_{n}^{J}(t):=S_{V}(t)\left(w_{0, n^{\prime}}^{J} w_{1, n}^{J}\right)$ satisfies

$$
\lim _{J \rightarrow \infty} \varlimsup_{n \rightarrow \infty}\left\|w_{n}^{J}\right\|_{L_{t}^{\infty} L_{x}^{p}(\mathbb{R}) \cap S(\mathbb{R})}=0, \quad \forall \frac{10}{3}<p \leq \infty .
$$

Moreover, we have the following Pythagorean expansion of the energy

$$
\mathcal{E}_{V}\left(\vec{u}_{n}\right)=\sum_{j=1}^{J} \mathcal{E}_{V}\left(\vec{U}_{L}^{j}\right)+\mathcal{E}_{V}\left(\vec{w}_{n}^{J}\right)+o(1),
$$

as $n \rightarrow \infty$.

The proof of of Lemma 5.4 is identical to the proof of Lemma 3.2 in [18] and we omit it. The sequence $\left\{\left(u_{0, n}, u_{1, n}\right)\right\}_{n}$ in Lemma 5.4 is said to have a profile decomposition with profiles $\left\{U_{L}^{j}\right\}_{j}$ and parameters $\left\{t_{j, n}\right\}_{j, n}$. We note that after passing to a further subsequence if necessary, we may assume that for all $j \geq 1$, either $t_{j, n}=0 \forall n$ or $\lim _{n} t_{j, n}= \pm \infty$.

In order to apply Lemma 5.4 in the context of the nonlinear problem (5.1), we will need the notion of nonlinear profiles. For each profile $U_{L}^{j}$ with time parameters $\left\{t_{j, n}\right\}_{n}$, we define its associated nonlinear profile $U^{j}$ to be the unique global solution to (5.1) such that

$$
\lim _{n \rightarrow \infty}\left\|\vec{U}^{j}\left(-t_{j, n}\right)-\vec{U}_{L}^{j}\left(-t_{j, n}\right)\right\|_{\mathcal{H}}=0 .
$$

It is easy to see that a nonlinear profile always exists. Indeed, if $t_{j, n}=0$ for all $n$, then we set $U^{j}$ to be the solution to (5.1) with initial data $\vec{U}^{j}(0)=\vec{U}_{L}^{j}(0)$. If $\lim _{n}-t_{j, n}=\infty$, say, then we set $U^{j}$ to be the unique globally defined solution to the integral equation

$$
U^{j}(t)=\vec{U}_{L}^{j}\left(-t_{j, n}\right)-\int_{t}^{\infty} S_{V}(t-s)\left(0, N\left(\cdot, U^{j}(s)\right)\right) d s
$$

A unique global solution to (5.29) can be shown to exist using contraction mapping arguments in the spirit of those used in Proposition 5.1 and Proposition 5.2 .

For each nonlinear profile $U^{j}$, we denote

$$
U_{n}^{j}(t):=U^{j}\left(t-t_{j, n}\right)
$$

Using Proposition 5.2, we obtain the following nonlinear profile decomposition from the linear profile decomposition in Lemma5.4.

Lemma 5.5 (Nonlinear Profile Decomposition). Let $\left\{\left(u_{0, n}, u_{1, n}\right)\right\}_{n}$ be a bounded sequence in $\mathcal{H}$ admitting a profile decomposition with profiles $\left\{U_{L}^{j}\right\}_{j}$ and parameters $\left\{t_{j, n}\right\}_{j, n}$. Let $T_{n} \in[0,+\infty)$. Assume

$$
\forall j \geq 1, \quad \limsup _{n \rightarrow \infty}\left\|U^{j}\right\|_{S\left(-t_{j, n}, T_{n}-t_{j, n}\right)}<\infty .
$$

Let $u_{n}$ be the unique global solution to (5.1) with initial data $\vec{u}(0)=\left(u_{0, n}, u_{1, n}\right)$. Then

$$
\limsup _{n \rightarrow \infty}\left\|u_{n}\right\|_{S\left(0, T_{n}\right)}<\infty,
$$


and for all $t \in\left[0, T_{n}\right]$

$$
\vec{u}_{n}(t)=\sum_{j=1}^{J} \vec{u}_{n}^{j}(t)+\vec{w}_{n}^{J}(t)+\vec{r}_{n}^{\prime}(t),
$$

with

$$
\lim _{J \rightarrow \infty} \varlimsup_{n \rightarrow \infty}\left[\left\|r_{n}^{J}\right\|_{S\left(0, T_{n}\right)}+\sup _{t \in\left[0, T_{n}\right]}\left\|\vec{r}_{n}^{\exists}(t)\right\|_{\mathcal{H}}\right]=0 .
$$

An analogous statement holds if $T_{n}<0$.

Proof. For $J \geq 1, n \geq 1$, define

$$
U_{n}^{J}(t):=\sum_{j=1}^{J} U_{n}^{j}(t)+w_{n}^{J}(t) .
$$

We will apply Proposition 5.2 with $U=U_{n}^{J}$ and $u=u_{n}$ for $n$ and $J$ large. We first show that

$$
\varlimsup_{J} \varlimsup_{n}\left\|U_{n}^{J}\right\|_{S\left(0, T_{n}\right)}<\infty \text {. }
$$

By assumption, there exists $M>0$ such that $\forall n,\left\|\left(u_{0, n}, u_{1, n}\right)\right\|_{\mathcal{H}}^{2} \simeq \mathcal{E}_{V}\left(u_{0, n}, u_{1, n}\right) \leq M$. The Pythagorean expansion of the energy (5.28) implies that

$$
\varlimsup_{J} \varlimsup_{n} \mathcal{E}_{V}\left(w_{n}^{J}\right)+\sum_{j=1}^{\infty} \mathcal{E}_{V}\left(U_{L}^{j}\right) \leq M .
$$

Hence, there exists $J_{0} \geq 1$ such that

$$
\sum_{j>J_{0}} \mathcal{E}_{V}\left(\vec{U}_{L}^{j}\right) \ll \delta^{2},
$$

where $\delta$ is from Proposition 5.1. In particular, this implies by Proposition 5.1 that the nonlinear profiles satisfy for all $j>J_{0}$

$$
\left\|\vec{U}^{j}\right\|_{L_{t}^{\infty} \mathcal{H}}+\left\|U^{j}\right\|_{S(\mathbb{R})}+\left\|U^{j}\right\|_{W(\mathbb{R})} \lesssim \mathcal{E}_{V}\left(\vec{U}_{L}^{j}\right)^{1 / 2}
$$

Let $J \geq 1$. Then

$$
\left\|U_{n}^{J}\right\|_{S\left(0, T_{n}\right)} \leq\left\|\sum_{j=1}^{J} U_{n}^{j}\right\|_{S\left(0, T_{n}\right)}+\left\|w_{n}^{J}\right\|_{S(\mathbb{R})} .
$$

Now

$$
\begin{aligned}
\left\|\sum_{j=1}^{J} U_{n}^{j}\right\|_{S\left(0, T_{n}\right)}^{3} & \leq\left\|\sum_{j=1}^{J}\right\| U_{n}^{j}\left\|_{L_{x}^{6}}\right\|_{L_{t}^{3}\left(0, T_{n}\right)}^{3} \\
& =\sum_{j=1}^{J}\left\|U_{n}^{j}\right\|_{S\left(0, T_{n}\right)}^{3}+\epsilon_{n}^{J},
\end{aligned}
$$

where the error $\epsilon_{n}^{J}$ is a sum of terms of the form

$$
\int_{0}^{T}\left\|U_{n}^{j}(t)\right\|_{L_{x}^{6}}\left\|U_{n}^{k}(t)\right\|_{L_{x}^{6}}\left\|U_{n}^{l}(t)\right\|_{L_{x}^{6}} d t
$$

with $1 \leq j, k, l \leq J$ and $j \neq k$. We claim that

$$
\lim _{n \rightarrow \infty} \int_{0}^{T_{n}}\left\|U_{n}^{j}(t)\right\|_{L_{x}^{6}}\left\|U_{n}^{k}(t)\right\|_{L_{x}^{6}}\left\|U_{n}^{l}(t)\right\|_{L_{x}^{6}} d t=0 .
$$


Indeed, by the assumption (5.30) and an approximation argument, we may assume that the functions $U^{j}, U^{k}$ are compactly supported in $t$. Now

$$
\begin{aligned}
\int_{0}^{T_{n}}\left\|U_{n}^{j}(t)\right\|_{L_{x}^{6}}\left\|U_{n}^{k}(t)\right\|_{L_{x}^{6}}\left\|U_{n}^{l}(t)\right\|_{L_{x}^{6}} d t & \lesssim\left(\int_{0}^{T_{n}}\left\|U_{n}^{j}(t)\right\|_{L_{x}^{6}}^{3 / 2}\left\|U_{n}^{k}(t)\right\|_{L_{x}^{6}}^{3 / 2} d t\right)^{2 / 3}\left\|U_{n}^{l}\right\|_{S\left(0, T_{n}\right)} \\
& \lesssim\left(\int_{0}^{T_{n}}\left\|U_{n}^{j}(t)\right\|_{L_{x}^{6}}^{3 / 2}\left\|U_{n}^{k}(t)\right\|_{L_{x}^{6}}^{3 / 2} d t\right)^{2 / 3} .
\end{aligned}
$$

Extending the integration over all of $\mathbb{R}$ and changing variables implies that

$$
\int_{0}^{T_{n}}\left\|U_{n}^{j}(t)\right\|_{L_{x}^{6}}^{3 / 2}\left\|U_{n}^{k}(t)\right\|_{L_{x}^{6}}^{3 / 2} d t \leq \int\left\|U^{j}(t)\right\|_{L_{x}^{6}}^{3 / 2}\left\|U^{k}\left(t+t_{j, n}-t_{k, n}\right)\right\|_{L_{x}^{6}}^{3 / 2} d t
$$

The orthogonality of the parameters implies that $\left|t_{j, n}-t_{k, n}\right| \rightarrow_{n} \infty$. Thus, the support of $U^{j}(\cdot)$ and $U^{k}\left(\cdot+t_{j, n}-t_{k, n}\right)$ are eventually disjoint whence

$$
\lim _{n \rightarrow \infty} \int\left\|U^{j}(t)\right\|_{L_{x}^{6}}^{3 / 2}\left\|U^{k}\left(t+t_{j, n}-t_{k, n}\right)\right\|_{L_{x}^{6}}^{3 / 2} d t=0 .
$$

This proves (5.34). Returning to (5.33) and recalling our choice of $J_{0}$, we see that

$$
\begin{aligned}
\varlimsup_{n}\left\|\sum_{j=1}^{J} U_{n}^{j}\right\|_{S\left(0, T_{n}\right)}^{3} & \leq \varlimsup_{n} \sum_{j=1}^{J}\left\|U_{n}^{j}\right\|_{S\left(0, T_{n}\right)}^{3} \\
& \lesssim \sum_{j=1}^{J_{0}} \varlimsup_{n}\left\|U_{n}^{j}\right\|_{S\left(0, T_{n}\right)}^{3}+\sum_{j>J_{0}}\left\|U^{j}\right\|_{S(\mathbb{R})}^{3} \\
& \lesssim 1+\sum_{j>J_{0}} \mathcal{E}_{V}\left(\vec{U}_{L}^{j}\right)^{3 / 2} \\
& \lesssim 1+M,
\end{aligned}
$$

where the implied constant is independent of $J$. Thus,

$$
\varlimsup_{J} \varlimsup_{n}\left\|U_{n}^{J}\right\|_{S\left(0, T_{n}\right)} \leq \varlimsup_{J} \varlimsup_{n}\left\|\sum_{j=1}^{J} U_{n}^{j}\right\|_{S\left(0, T_{n}\right)}+\varlimsup_{J} \varlimsup_{n}\left\|w_{n}^{J}\right\|_{S(\mathbb{R})}<\infty .
$$

Using similar arguments, we also conclude that

$$
\varlimsup_{J} \varlimsup_{n}\left\|U_{n}^{J}\right\|_{L_{t}^{\infty} \mathcal{H}\left(0, T_{n}\right)}<\infty .
$$

We now verify that the following error

$$
\begin{aligned}
e_{n}^{J} & :=\partial_{t}^{2} U_{n}^{J}-\Delta_{g} U_{n}^{J}+V U_{n}^{J}-N\left(\cdot, U_{n}^{J}\right) \\
& =\sum_{j=1}^{J} N\left(\cdot, U_{n}^{j}\right)-N\left(\cdot, \sum_{j=1}^{J} U_{n}^{j}+w_{n}^{J}\right),
\end{aligned}
$$

satisfies

$$
\varlimsup_{J} \varlimsup_{n}\left\|e_{n}^{J}\right\|_{N\left(0, T_{n}\right)}=0 .
$$

We focus only on the quadratic part of $N(\cdot, u)$ since the other parts can be handled similarly. More precisely, we show that

$$
\varlimsup_{J} \varlimsup_{n}\left\|\left(2\langle r\rangle^{-1} \sin 2 Q\right)\left(\sum_{j=1}^{J}\left(U_{n}^{j}\right)^{2}-\left(\sum_{j=1} U_{n}^{j}+w_{n}^{J}\right)^{2}\right)\right\|_{L_{t}^{3 / 2} \dot{W}_{x}^{1 / 2,3 / 2}\left(0, T_{n}\right)}=0
$$


To lessen the notation, for $I \subseteq \mathbb{R}$, we denote $W^{\prime}(I):=L_{t}^{3 / 2} \dot{W}_{x}^{1 / 2,3 / 2}(I)$. We observe that

$$
\begin{aligned}
& \left\|\left(2\langle r\rangle^{-1} \sin 2 Q\right)\left(\sum_{j=1}^{J}\left(U_{n}^{j}\right)^{2}-\left(\sum_{j=1} U_{n}^{j}+w_{n}^{J}\right)^{2}\right)\right\|_{W^{\prime}\left(0, T_{n}\right)} \\
& \lesssim\left\|\left(2\langle r\rangle^{-1} \sin 2 Q\right) w_{n}^{J} \sum_{j=1}^{J} U_{n}^{j}\right\|_{W^{\prime}\left(0, T_{n}\right)}+\sum_{j \neq k}\left\|\left(2\langle r\rangle^{-1} \sin 2 Q\right) U_{n}^{j} \sum_{j=1}^{J} U_{n}^{k}\right\|_{W^{\prime}\left(0, T_{n}\right)} \\
& +\left\|\left(2\langle r\rangle^{-1} \sin 2 Q\right)\left(w_{n}^{J}\right)^{2}\right\|_{W^{\prime}\left(0, T_{n}\right)} \\
& =: A_{n}^{J}+B_{n}^{J}+C_{n}^{J} .
\end{aligned}
$$

Using the orthogonality of the parameters and arguments as in the previous paragraph, it is straightforward to show that

$$
\lim _{n} B_{n}^{J}=0 .
$$

To estimate $C_{n}^{J}$, we recall that $\varlimsup_{j} \varlimsup_{n}\left\|w_{n}^{J}\right\|_{S(\mathbb{R})}=0$ and $\vec{w}_{n}^{J}(0)$ is bounded in $\mathcal{H}$. Thus, by the product rule (see the proof of Proposition 5.1) and Strichartz estimates, we have

$$
C_{n}^{J} \lesssim\left\|w_{n}^{J}\right\|_{S(\mathbb{R})}\left\|w_{n}^{J}\right\|_{W(\mathbb{R})}+\left\|w_{n}^{J}\right\|_{S(\mathbb{R})}^{2} \lesssim\left\|w_{n}^{J}\right\|_{S(\mathbb{R})}+\left\|w_{n}^{J}\right\|_{S(\mathbb{R})}^{2}
$$

whence $\varlimsup_{\lim } \varlimsup_{n} C_{n}^{J}=0$. We now show that $\varlimsup_{J} \varlimsup_{J} A_{n}^{J}=0$. Let $\epsilon>0$. By the arguments used to show that $\varlimsup_{J} \varlimsup_{n}\left\|U_{n}^{J}\right\|_{S\left(0, T_{n}\right)}<\infty$, there exists $J_{1}=J_{1}(\epsilon)>J_{0}$ such that for all $J>J_{1}$

$$
\varlimsup_{n}\left(\left\|\sum_{j=J_{1}+1}^{J} U_{n}^{j}\right\|_{S\left(0, T_{n}\right)}+\left\|\sum_{j=J_{1}+1}^{J} U_{n}^{j}\right\|_{S\left(0, T_{n}\right)}\right)<\epsilon .
$$

Thus, by the product rule, we obtain

$$
\begin{aligned}
& \varlimsup_{n}\left\|\left(2\langle r\rangle^{-1} \sin 2 Q\right) w_{n}^{J} \sum_{j=J_{1}+1}^{J} U_{n}^{j}\right\|_{W^{\prime}\left(0, T_{n}\right)} \\
& \lesssim \varlimsup_{n}\left\|w_{n}^{J}\right\|_{W(\mathbb{R})}\left\|\sum_{j=J_{1}+1}^{J} U_{n}^{j}\right\|_{S\left(0, T_{n}\right)}+\varlimsup_{n}\left\|w_{n}^{J}\right\|_{S(\mathbb{R})}\left\|\sum_{j=J_{1}+1}^{J} U_{n}^{j}\right\|_{S\left(0, T_{n}\right)} \\
& +\varlimsup_{n}\left\|w_{n}^{J}\right\|_{S(\mathbb{R})}\left\|\sum_{j=J_{1}+1}^{J} U_{n}^{j}\right\|_{W\left(0, T_{n}\right)} \\
& \lesssim \epsilon,
\end{aligned}
$$

where the implied constant is independent of $J$. Thus,

$$
\varlimsup_{J} \varlimsup_{n} A_{n}^{J} \lesssim \epsilon+\varlimsup_{J} \varlimsup_{n} \sum_{j=1}^{J_{1}}\left\|\left(2\langle r\rangle^{-1} \sin 2 Q\right) w_{n}^{J} U_{n}^{j}\right\|_{W^{\prime}\left(0, T_{n}\right)} .
$$

Fix $j \in\left\{1, \ldots, J_{1}\right\}$. We wish to show that

$$
\varlimsup_{J} \varlimsup_{n} \sum_{j=1}^{J_{1}}\left\|\left(2\langle r\rangle^{-1} \sin 2 Q\right) w_{n}^{J} U_{n}^{j}\right\|_{W^{\prime}\left(0, T_{n}\right)}=0 .
$$

By the product rule,

$$
\left\|\left(2\langle r\rangle^{-1} \sin 2 Q\right) w_{n}^{J} U_{n}^{j}\right\|_{L_{x}^{3 / 2}} \lesssim\left\|w_{n}^{J}\right\|_{L_{x}^{6}}\left\|U_{n}^{j}\right\|_{L_{x}^{6}}+\left\|w_{n}^{J}\right\|_{L_{x}^{6}}\left\|U_{n}^{j}\right\|_{\dot{W}_{x}^{1 / 2,3}}+\left\|w_{n}^{J}\right\|_{\dot{W}_{x}^{1 / 2,3}}\left\|U_{n}^{j}\right\|_{L_{x}^{6}}
$$


Arguing as in the proof of Proposition 5.2, the assumption (5.30) also implies that for all $j \geq 1$,

$$
\varlimsup_{n}\left\|U^{j}\right\|_{W\left(0, T_{n}\right)}<\infty .
$$

This fact, (5.41), Hölder's inequality, and the fact that $\varlimsup_{J} \varlimsup_{n}\left\|w_{n}^{J}\right\|_{S\left(0, T_{n}\right)}=0$ imply that

$$
\varlimsup_{J} \varlimsup_{n}\left\|\left(2\langle r\rangle^{-1} \sin 2 Q\right) w_{n}^{J} U_{n}^{j}\right\|_{W^{\prime}\left(0, T_{n}\right)} \lesssim \varlimsup_{J} \varlimsup_{n}\|\| w_{n}^{J}(t)\left\|_{\dot{W}_{x}^{1 / 2,3}}\right\| U_{n}^{j}(t)\left\|_{L_{x}^{6}}\right\|_{L_{t}^{3 / 2}\left(0, T_{n}\right)} .
$$

We now show that

$$
\varlimsup_{J} \varlimsup_{n} \int_{0}^{T_{n}}\left\|w_{n}^{J}(t)\right\|_{\dot{W}_{x}^{1 / 2,}}^{3 / 2}\left\|U_{n}^{j}(t)\right\|_{L_{x}^{6}}^{3 / 2} d t=0 .
$$

By the assumption (5.30) and an approximation argument, we can assume that $U^{j}$ is compactly supported in $t$. By interpolation, we have the estimate

$$
\forall t, \quad\left\|w^{J}(t)\right\|_{\dot{W}_{x}^{1 / 2,3}} \lesssim\left\|\nabla w_{n}^{J}(t)\right\|_{L_{x}^{2}}^{1 / 2}\left\|w_{n}^{J}(t)\right\|_{L_{x}^{6}}^{1 / 2} .
$$

Thus, by Hölder's inequality

$$
\begin{aligned}
\int_{0}^{T_{n}}\left\|w_{n}^{J}(t)\right\|_{\dot{W}_{x}^{1 / 2,3}}^{3 / 2}\left\|U_{n}^{j}(t)\right\|_{L_{x}^{6}}^{3 / 2} d t & \lesssim \int\left\|w_{n}^{J}\left(t+t_{j, n}\right)\right\|_{\dot{W}_{x}^{1 / 2,3}}^{3 / 2}\left\|U^{j}(t)\right\|_{L_{x}^{6}}^{3 / 2} d t \\
& \lesssim \int\left\|\vec{w}_{n}^{J}\right\|_{L_{t}^{\infty} \mathcal{H}^{\prime / 4}}^{3 / w_{n}^{J}}\left(t+t_{j, n}\right)\left\|_{L_{x}^{6}}^{3 / 4}\right\| U^{j}(t) \|_{L_{x}^{6}}^{3 / 2} d t \\
& \lesssim\left\|w_{n}^{J}\right\|_{S(\mathbb{R})^{\prime}}^{3 / 4}
\end{aligned}
$$

where the implied constant depends on $U^{j}$. Thus,

$$
\varlimsup_{J} \varlimsup_{n} \int_{0}^{T_{n}}\left\|w_{n}^{J}(t)\right\|_{\dot{\mathrm{W}}_{x}^{1 / 2,3}}^{3 / 2}\left\|U_{n}^{j}(t)\right\|_{L_{x}^{6}}^{3 / 2} d t \lesssim \varlimsup_{J} \varlimsup_{n}\left\|w_{n}^{J}\right\|_{S(\mathbb{R})}^{3 / 4}=0 .
$$

This proves (5.44). By (5.43), this also proves (5.40). By (5.39), this proves

$$
\varlimsup_{J} \varlimsup_{n} A_{n}^{J} \lesssim \epsilon,
$$

which proves (5.36).

We have now demonstrated that the function $U_{n}^{J}$ satisfies the hypotheses stated in Proposition 5.2 uniformly in $J, n$ large and

$$
\varlimsup_{J} \varlimsup_{n}\left\|e_{n}^{J}\right\|_{N\left(0, T_{n}\right)}=0 .
$$

Since $\vec{U}_{n}^{J}(0)=u_{n}(0)+o_{\mathcal{H}}(1)$ as $n \rightarrow \infty$, we have by Proposition [5.2, for $t \in\left[0, T_{n}\right]$,

$$
\vec{u}_{n}(t)=\vec{U}_{n}^{J}(t)+\vec{r}_{n}^{J}(t),
$$

with

$$
\lim _{J \rightarrow \infty} \varlimsup_{n \rightarrow \infty}\left[\left\|r_{n}^{J}\right\|_{S\left(0, T_{n}\right)}+\sup _{t \in\left[0, T_{n}\right]}\left\|\vec{r}_{n}^{\exists}(t)\right\|_{\mathcal{H}}\right]=0 .
$$

This completes the proof.

We now prove Proposition 5.3

Proof of Proposition 5.3 For $A>0$, define

$$
\mathcal{B}(A):=\left\{\left(u_{0}, u_{1}\right) \in \mathcal{H}: \text { if } u \text { solves (5.1) with } \vec{u}(0)=\left(u_{0}, u_{1}\right) \text { then } \sup _{t \in[0, \infty)} \mathcal{E}_{V}\left(\vec{u}_{n}(t)\right)^{1 / 2} \leq A\right\} .
$$


We say that the property $\mathcal{S C}(A)$ holds if for all $\left(u_{0}, u_{1}\right) \in \mathcal{B}(A)$, the solution $u$ to (5.1) satisfies $\|u\|_{S(0, \infty)}<\infty$. Note that by Proposition 5.1 and (5.10), every solution $u$ to (5.1) is in $\mathcal{B}(A)$ for some $A$ and if $0<A<\delta$, where $\delta$ is as in Proposition 5.1, then $\mathcal{S C}(A)$ holds. Define

$$
A_{C}:=\sup \{A>0: \mathcal{S C}(A) \text { holds. }\}>0 .
$$

By the temporal symmetry of (5.1) and Proposition 5.1, we see that Theorem 4.1]is equivalent to the statement

$$
A_{C}=\infty .
$$

Suppose not, i.e. $0<A_{C}<\infty$. Then there exists a sequence of real numbers $A_{n} \downarrow A$ and a sequence $\left\{\left(u_{0, n}, u_{1, n}\right)\right\}_{n}$ in $\mathcal{H}$ such that the corresponding solutions $u_{n}$ to (5.1) with initial data $\vec{u}_{n}(0)=\left(u_{0, n}, u_{1, n}\right)$ satisfy

$$
\begin{aligned}
\exists T_{n}<0, T_{n} \rightarrow-\infty, \sup _{t \in\left(T_{n}, \infty\right)} \mathcal{E}_{V}\left(\vec{u}_{n}(t)\right)^{1 / 2} & \leq A_{n}, \\
\left\|u_{n}\right\|_{S(0, \infty)} & =\infty, \\
\lim _{n \rightarrow \infty}\left\|u_{n}\right\|_{S\left(-T_{n}, 0\right)} & =\infty .
\end{aligned}
$$

Note that (5.46) and (5.10) imply that the sequence $\left\{\vec{u}_{n}(0)=\left(u_{0, n}, u_{1, n}\right)\right\}_{n}$ is bounded in $\mathcal{H}$. After passing to a subsequence if necessary, $\vec{u}_{n}(0)$ admits a profile decomposition

$$
\vec{u}_{n}(0)=\sum_{j=1}^{J} \vec{u}_{L}^{j}\left(-t_{j, n}\right)+\vec{w}_{n}^{J}(0)
$$

with profiles $\left\{U_{L}^{j}\right\}_{j}$ and time parameters $\left\{t_{j, n}\right\}_{j, n}$ by Lemma 5.4. As before we assume, without loss of generality, that for all $j$ either $t_{j, n}=0 \forall n$ or $\lim _{n} t_{j, n}= \pm \infty$. Let $\left\{U^{j}\right\}_{j}$ be the sequence of associated nonlinear profiles. By the Pythagorean expansion of the energy, there exists $J_{0}>1$ such that

$$
\sum_{j>J_{0}} \mathcal{E}_{V}\left(\vec{U}_{L}^{j}\right) \ll \delta^{2}
$$

where $\delta$ is as in the small data theory, Proposition 5.1. Thus, the associated nonlinear profiles satisfy

$$
\left\|U^{j}\right\|_{S(\mathbb{R})} \lesssim \mathcal{E}_{V}\left(\vec{U}_{L}^{j}\right)^{1 / 2} .
$$

Define

$$
\mathcal{J}=\left\{j \in\left\{1, \ldots, J_{0}\right\}:\left\|U^{j}\right\|_{S(0, \infty)}=\infty\right\} .
$$

First, we note that $\mathcal{J} \neq \varnothing$. Otherwise, by the definition of nonlinear profiles and our choice of $J_{0}$, we have

$$
\forall j \geq 1, \quad\left\|U^{j}\right\|_{S(0, \infty)}<\infty .
$$

By Lemma 5.5, this would imply that $\left\|u_{n}\right\|_{S(0, \infty)}<\infty$ for large $n$, a contradiction to $(\underline{5.46)}$. Thus, $\mathcal{J} \neq \varnothing$. Note that if $j \in \mathcal{J}$ and $-t_{j, n} \rightarrow_{n} \infty$, then $U^{j}$ scatters forward in time, i.e. $\left\|U^{j}\right\|_{S(0, \infty)}<\infty$, a contradiction to our definition of $\mathcal{J}$. Thus, for all $j \in \mathcal{J}$, we have that $-t_{j, n} \rightarrow_{n}-\infty$. By the orthogonality of the parameters and after rearranging the first $J_{0}$ profiles if necessary, we may assume that if $j>1$, then

$$
\lim _{n \rightarrow \infty} t_{1, n}-t_{j, n}=-\infty
$$

We now claim that $\mathcal{J}=\{1\}$ and that for all $j \geq 2, \vec{U}_{L}^{j}=0$. Suppose not and, say, $\vec{U}_{L}^{2} \neq 0$. Then for $T \geq 0$ and for all $j \geq 1$,

$$
\varlimsup_{n}\left\|U^{j}\right\|_{S\left(-t_{j, n}, T+t_{1, n}-t_{j, n}\right)}<\infty .
$$


By Proposition 5.5, the Pythagorean expansion of the energy, and conservation of the energy $\mathcal{E}_{V}(\cdot)$ we conclude that

$$
\begin{aligned}
\mathcal{E}_{V}\left(\vec{u}_{n}\left(T+t_{1, n}\right)\right) & =\mathcal{E}_{V}\left(\vec{U}^{1}(T)\right)+\sum_{j=2}^{J} \mathcal{E}_{V}\left(\vec{U}^{j}\left(T+t_{1, n}-t_{j, n}\right)\right)+\mathcal{E}_{V}\left(\vec{w}_{n}^{J}\right)+\mathcal{E}_{V}\left(\vec{r}_{n}^{J}\left(T+t_{1, n}\right)\right)+o_{n}(1) \\
& =\mathcal{E}_{V}\left(\vec{U}^{1}(T)\right)+\sum_{j=2}^{J} \mathcal{E}_{V}\left(\vec{U}_{L}^{j}\right)+\mathcal{E}_{V}\left(\vec{w}_{n}^{J}\right)+\mathcal{E}_{V}\left(\vec{r}_{n}^{J}\left(T+t_{1, n}\right)\right)+o_{n}(1) \\
& \geq \mathcal{E}_{V}\left(\vec{U}^{1}(T)\right)+\mathcal{E}_{V}\left(\vec{U}_{L}^{2}\right)+o_{n}(1)
\end{aligned}
$$

as $n \rightarrow \infty$. In particular,

$$
\mathcal{E}_{V}\left(\vec{U}^{1}(T)\right) \leq A_{0}^{2}<A_{C}^{2} .
$$

Since $T \geq 0$ was arbitrary, we conclude that $\sup _{t \in[0, \infty)} \mathcal{E}_{V}\left(\vec{U}^{1}(t)\right)^{1 / 2} \leq A_{0}<A_{C}$. By the minimality of $A_{C}$, it follows that $\left\|U^{1}\right\|_{S(0, \infty)}<\infty$, a contradiction to the fact that $1 \in \mathcal{J}$. Thus, $\vec{U}_{L}^{j}=0$ for all $j \geq 2$. By a similar argument, we also deduce that

$$
\lim _{n \rightarrow \infty} \mathcal{E}_{V}\left(\vec{w}_{n}^{1}\right)=0
$$

or equivalently $\lim _{n}\left\|\vec{w}_{n}^{J}(0)\right\|_{\mathcal{H}}=0$.

We have now shown that

$$
\vec{u}_{n}(0)=\vec{U}^{1}\left(-t_{1, n}\right)+o_{\mathcal{H}}(1),
$$

as $n \rightarrow \infty$. We claim that $t_{1, n}=0$ for all $n$. If not, then by our initial assumptions on the parameters we have $-t_{1, n} \rightarrow_{n}-\infty$. This implies that $\left\|U^{1}\right\|_{S(-\infty, 0)}<\infty$. By Proposition 5.2 , we deduce that $\varlimsup_{\lim }\left\|u_{n}\right\|_{S(-\infty, 0)}<\infty$, a contradiction to (5.46). Thus,

$$
\vec{u}_{n}(0)=\vec{U}^{1}(0)+o_{\mathcal{H}}(1),
$$

as $n \rightarrow \infty$. Define $u_{*}=U^{1}$. Then by Proposition 5.2 and (5.46), $u_{*}$ satisfies

$$
\begin{array}{r}
\sup _{t \in(-\infty, \infty)} \mathcal{E}_{V}\left(\vec{u}_{*}(t)\right) \leq A_{C}, \\
\left\|u_{*}\right\|_{S(-\infty, 0)}=\left\|u_{*}\right\|_{S(0, \infty)}=\infty .
\end{array}
$$

Finally, we show that $\left\{\vec{u}_{*}(t): t \in \mathbb{R}\right\}$ is precompact in $\mathcal{H}$. By continuity of the flow, it suffices to show that if $\left\{t_{n}\right\}_{n}$ is a sequence in $\mathbb{R}$, with $\lim _{n \rightarrow \infty} t_{n}= \pm \infty$, then there exists a subsequence (still denoted by $t_{n}$ ) such that $\vec{u}_{*}\left(t_{n}\right)$ converges in $\mathcal{H}$. Suppose that $t_{n} \rightarrow_{n} \infty$. Define $\left(u_{0, n}, u_{1, n}\right):=\vec{u}_{*}\left(t_{n}\right)$. Then the solution $u_{n}$ to (5.1) with initial data $\vec{u}_{n}(0)=\left(u_{0, n}, u_{1 . n}\right)$ is given by $u_{n}(t)=u_{*}\left(t+t_{n}\right)$ whence by (5.48), the solutions $u_{n}$ satisfy the conditions given in (5.46). Thus, we may repeat the previous argument to conclude that there exists a subsequence (still indexed by $n$ ) and $\vec{U}^{1}(0) \in \mathcal{H}$ such that

$$
u_{*}\left(t_{n}\right)=\vec{u}_{n}(0)=\vec{U}^{1}(0)+o_{\mathcal{H}}(1),
$$

as $n \rightarrow \infty$. If $t_{n} \rightarrow-\infty$, then we apply the previous argument to $u_{*}(-t)$ to conclude. Thus, the set

$$
K:=\left\{\vec{u}_{*}(t): t \in \mathbb{R}\right\},
$$

is precompact in $\mathcal{H}$. This completes the proof.

\section{Rigidity TheOREM}

In this section, we show that the critical element from Proposition 5.3 does not exist and conclude the proof of our main result Theorem 4.1 (equivalently Theorem 1.1). In particular, we prove the following. 
Proposition 6.1. Let $u$ be a global solution of (5.1) such that the trajectory

$$
K=\{\vec{u}(t): t \in \mathbb{R}\},
$$

is precompact in $\mathcal{H}:=\mathcal{H}\left((-\infty, \infty) ;\left(r^{2}+1\right)^{2} d r\right)$. Then $\vec{u}=(0,0)$.

We first note that for a solution $u$ as in Proposition 6.1, we have the following uniform control of the energy in exterior regions.

Lemma 6.2. Let $u$ be as in Proposition 6.1] Then we have

$$
\begin{aligned}
\forall R \geq 0, \quad \lim _{|t| \rightarrow \infty}\|\vec{u}(t)\|_{\mathcal{H}\left(|r| \geq R+|t| ;\left(r^{2}+1\right)^{2} d r\right)} & =0, \\
\lim _{R \rightarrow \infty}\left[\sup _{t \in \mathbb{R}}\|\vec{u}(t)\|_{\mathcal{H}\left(|r| \geq R+|t| ;\left(r^{2}+1\right)^{2} d r\right)}\right] & =0 .
\end{aligned}
$$

To prove that $\vec{u}=(0,0)$, we will show that $u$ is a finite energy static solution to (5.1).

Proposition 6.3. Let $u$ be as in Proposition 6.1 Then there exists a static solution $U$ to (5.1) such that $\vec{u}=(U, 0)$.

We will first show that $\vec{u}$ is equal to static solutions $\left(U_{ \pm}, 0\right)$ to (5.1) on $\pm r>0$. Since the proof for $r<0$ is nearly identical, we only consider the case $r>0$.

Proposition 6.4. Let $u$ be as in Lemma6.1] Then there exists a static solution $U_{+}$to (5.1) such that $\vec{u}(t, r)=\left(U_{+}(r), 0\right)$ for all $t \in \mathbb{R}$ and all $r>0$.

6.1. Proof of Proposition 6.4. Let $\eta>0$ and let $u$ be as in Proposition 6.1. We will first show that $\vec{u}(0, r)=$ $\left(U_{+}(r), 0\right)$ on $r \geq \eta$ for some static solution $U_{+}$to (5.1).

We now introduce a function that will be integral in the proof. Define

$$
u_{e}(t, r):=\frac{r^{2}+1}{r^{2}} u(t, r), \quad(t, r) \in \mathbb{R} \times(0, \infty) .
$$

If $u$ solves (5.1), then $u_{e}$ solves

$$
\partial_{t}^{2} u_{e}-\partial_{r}^{2} u_{e}-\frac{4}{r} \partial_{r} u_{e}+V_{e}(r) u_{e}=N_{e}\left(r, u_{e}\right), \quad t \in \mathbb{R}, r>0,
$$

where

$$
V_{e}(r)=V(r)-\frac{2}{r^{2}\left(r^{2}+1\right)}
$$

and $N_{e}\left(r, u_{e}\right)=F_{e}\left(r, u_{e}\right)+G_{e}\left(r, u_{e}\right)$ where

$$
\begin{aligned}
& F_{e}\left(r, u_{e}\right)=\frac{r^{2}+1}{r^{2}} F\left(r, \frac{r^{2}}{r^{2}+1} u_{e}\right), \\
& G_{e}\left(r, u_{e}\right)=\frac{r^{2}+1}{r^{2}} G\left(r, \frac{r^{2}}{r^{2}+1} u_{e}\right) .
\end{aligned}
$$

Note that for all $R>0$, we have

$$
\left\|\vec{u}_{e}(t)\right\|_{\mathcal{H}\left(r \geq R ; r^{4} d r\right)} \leq C(R)\|\vec{u}\|_{\mathcal{H}\left(r \geq R ;\left(r^{2}+1\right)^{2} d r\right)}
$$

so that by Lemma6.2, $u_{e}$ inherits the compactness properties

$$
\begin{array}{r}
\forall R>0, \quad \lim _{|t| \rightarrow \infty}\left\|\vec{u}_{e}(t)\right\|_{\mathcal{H}\left(r \geq R+|t| ; r^{4} d r\right)}=0, \\
\lim _{R \rightarrow \infty}\left[\sup _{t \in \mathbb{R}}\left\|\vec{u}_{e}(t)\right\|_{\mathcal{H}\left(r \geq R+|t| ; r^{4} d r\right)}\right]=0 .
\end{array}
$$

We also note that due to (4.7)-(4.10) and the definition of $V_{e}, F_{e}$, and $G_{e}$, we have for all $r>0$,

$$
\begin{aligned}
\left|V_{e}(r)\right| & \lesssim r^{-4}, \\
\left|F_{e}\left(u_{e}, r\right)\right| & \lesssim r^{-3}\left|u_{e}\right|^{2}, \\
\left|G_{e}\left(u_{e}, r\right)\right| & \lesssim\left|u_{e}\right|^{3},
\end{aligned}
$$


where the implied constants depend on the harmonic map $Q$.

The proof that $\vec{u}=\left(U_{+}, 0\right)$ on $r \geq \eta$ for some $U_{+}$is split into three main steps. In the first two steps, we determine the precise asymptotics of the associated "Euclidean" solution $u_{e, 0}(r):=u_{e}(0, r), u_{e, 1}(r):=\partial_{t} u_{e}(0, r)$, as $r \rightarrow \infty$. In particular, we show that there exists $\alpha \in \mathbb{R}$ such that

$$
\begin{aligned}
r^{3} u_{e, 0}(r) & =\alpha+O\left(r^{-1}\right), \\
r \int_{r}^{\infty} u_{e, 1}(\rho) \rho d \rho & =O\left(r^{-1}\right),
\end{aligned}
$$

as $r \rightarrow \infty$. In the final step, we use this information to conclude the argument. For the remainder of this subsection we denote $\mathcal{H}\left(r \geq R ; r^{4} d r\right)$ simply by $\mathcal{H}(r \geq R)$ and the exterior region $\mathbb{R}^{5} \backslash B(0, \eta)$ by $\mathbb{R}_{*}^{5}$.

The key tool for establishing (6.11) and (6.12) is the following exterior energy estimate for radial free waves on Minkowski space $\mathbb{R}^{1+5}$.

Proposition 6.5 (Proposition $4.1[14])$. Let $v$ be a radial solution to the free wave equation in $\mathbb{R}^{1+5}$

$$
\begin{aligned}
& \partial_{t}^{2} v-\Delta v=0, \quad(t, x) \in \mathbb{R}^{1+5}, \\
& \vec{v}(0)=(f, g) \in \dot{H}^{1} \times L^{2}\left(\mathbb{R}^{5}\right) .
\end{aligned}
$$

Then for any $R>0$,

$$
\max _{ \pm} \inf _{ \pm t \geq 0} \int_{r \geq R+|t|}\left|\nabla_{t, x} v(t, r)\right|^{2} r^{4} d r \geq \frac{1}{2}\left\|\pi_{R}^{\perp}(f, g)\right\|_{\mathcal{H}\left(r \geq R ; r^{4} d r\right)}
$$

where $\pi_{R}=I-\pi_{R}^{\perp}$ is the orthogonal projection onto the plane

$$
P(R)=\operatorname{span}\left\{\left(r^{-3}, 0\right),\left(0, r^{-3}\right)\right\}
$$

in $\mathcal{H}(r \geq R)$. The left-hand side of (6.13) is identically 0 for data satisfying $(f, g)=\left(\alpha r^{-3}, \beta r^{-3}\right)$ for on $r \geq R$.

We remark here that Proposition 6.5 states, quantitatively, that generic solutions to the free wave equation on $\mathbb{R}^{1+5}$ emit a fixed amount of energy into regions exterior to light cones. However, this property is very sensitive to dimension and in fact fails in the case $R=0$ for general data $(f, g)$ in even dimensions (see [5]). Proposition 6.5 has been generalized to all odd dimensions $d \geq 3$ in the work [15]. We note that the orthogonal projections $\pi_{R}, \pi_{R}^{\perp}$ are given by

$$
\begin{aligned}
& \pi_{R}(f, 0)=R^{3} r^{-3} f(R), \quad \pi_{R}(0, g)=R r^{-3} \int_{R}^{\infty} g(\rho) \rho d \rho \\
& \pi_{R}^{\perp}(f, 0)=f(r)-R^{3} r^{-3} f(R), \quad \pi_{R}^{\perp}(0, g)=g(r)-R r^{-3} \int_{R}^{\infty} g(\rho) \rho d \rho,
\end{aligned}
$$

and thus we have

$$
\begin{aligned}
\left\|\pi_{R}(f, g)\right\|_{\dot{H}^{1} \times L^{2}(r>R)}^{2}= & 3 R^{3} f^{2}(R)+R\left(\int_{R}^{\infty} r g(r) d r\right)^{2}, \\
\left\|\pi_{R}^{\perp}(f, g)\right\|_{H^{1} \times L^{2}(r>R)}^{2}= & \int_{R}^{\infty} f_{r}^{2}(r) r^{4} d r-3 R^{3} f^{2}(R) \\
& +\int_{R}^{\infty} g^{2}(r) r^{4} d r-a\left(\int_{R}^{\infty} r g(r) d r\right)^{2} .
\end{aligned}
$$

We now proceed to the first step in proving Proposition 6.3

6.1.1. Step 1: Estimate for $\pi_{R}^{\perp} \overrightarrow{\mathcal{u}}_{e}$ in $\mathcal{H}(r \geq R)$. The first step in proving Proposition 6.3 is the following decay estimate for $\pi_{R}^{\perp} \vec{u}_{e}(t)$.

Lemma 6.6. There exists $R_{0}>1$ such that for all $R \geq R_{0}$ and for all $t \in \mathbb{R}$ we have

$$
\left\|\pi_{R}^{\perp} \vec{u}_{e}(t)\right\|_{\mathcal{H}(r \geq R)}^{2} \lesssim R^{-10 / 3}\left\|\pi_{R} \vec{u}_{e}(t)\right\|_{\mathcal{H}(r \geq R)}^{2}+R^{-22 / 6}\left\|\pi_{R} \vec{u}_{e}(t)\right\|_{\mathcal{H}(r \geq R)}^{4}+\left\|\pi_{R} \vec{u}_{e}(t)\right\|_{\mathcal{H}(r \geq R)}^{6} .
$$


Since we are only interested in the behavior of $u$ in exterior regions $\{r \geq R+|t|\}$, we first consider a modified Cauchy problem. In particular, we can, by finite speed of propagation, alter $V_{e}, F_{e}$, and $G_{e}$ in (6.2) on the interior region $\{1 \leq r \leq R+|t|\}$ without affecting the behavior of $\vec{u}_{e}$ on the exterior region $\{r \geq R+|t|\}$.

Definition 6.7. For a function $f=f(r, u): \mathbb{R}_{*}^{5} \times \mathbb{R} \rightarrow \mathbb{R}$, we define for $R \geq \eta$,

$$
f_{R}(t, r, u):=\left\{\begin{array}{ll}
f(R+|t|, u) & \text { if } \eta \leq r \leq R+|t|, \\
f(r, u) & \text { if } r \geq R+|t| .
\end{array} .\right.
$$

We now consider solutions to a modified version of (6.2):

$$
\begin{aligned}
& \partial_{t}^{2} h-\partial_{r}^{2} h-\frac{4}{r} \partial_{r} h=N_{R}(t, r, h), \quad(t, r) \in \mathbb{R} \times \mathbb{R}_{*}^{5}, \\
& \vec{h}(0)=\left(h_{0}, h_{1}\right) \in \mathcal{H}_{0}(r \geq \eta),
\end{aligned}
$$

where $\mathcal{H}_{0}(r \geq \eta)=\left\{\left(u_{0}, u_{1}\right) \in \mathcal{H}(r \geq \eta): u_{0}(\eta)=0\right\}$ and

$$
N_{R}(t, r, h)=-V_{e, R}(t, r) h+F_{e, R}(t, r, h)+G_{e}(t, r, h) .
$$

We note that from Definition 6.7 and (6.8), (6.9), and (6.10), we have that

$$
\begin{aligned}
\left|V_{e, R}(t, r)\right| & \lesssim \begin{cases}(R+|t|)^{-4} & \text { if } \eta \leq r \leq R+|t|, \\
r^{-4} & \text { if } r \geq R+|t|,\end{cases} \\
\left|F_{e, R}(t, r, h)\right| & \lesssim \begin{cases}(R+|t|)^{-3}|h|^{2} & \text { if } \eta \leq r \leq R+|t|, \\
r^{-3}|h|^{3} & \text { if } r \geq R+|t|,\end{cases} \\
\left|G_{e}(r, h)\right| & \lesssim|h|^{3},
\end{aligned}
$$

Lemma 6.8. There exist $R_{0}>0$ large and $\delta_{0}>0$ small such that for all $R \geq R_{0}$ and all $\left(h_{0}, h_{1}\right) \in \mathcal{H}_{0}(r \geq \eta)$ with

$$
\left\|\left(h_{0}, h_{1}\right)\right\|_{\mathcal{H}_{0}(r \geq \eta)} \leq \delta_{0},
$$

there exists a unique globally defined solution $h$ to (6.18) such that

$$
\|h\|_{L_{t}^{3} L_{x}^{6}\left(\mathbb{R} \times \mathbb{R}_{*}^{5}\right)} \lesssim\|\vec{h}(0)\|_{\mathcal{H}(r \geq \eta)} .
$$

Moreover, if we define $h_{L}$ to be the solution to the free equation $\partial_{t}^{2} h_{L}-\Delta h_{L}=0,(t, x) \in \mathbb{R} \times \mathbb{R}_{*}^{5}, \vec{h}_{L}(0)=\left(h_{0}, h_{1}\right)$, then

$$
\sup _{t \in \mathbb{R}}\left\|\vec{h}(t)-\vec{h}_{L}(t)\right\|_{\mathcal{H}(r \geq \eta)} \lesssim R^{-5 / 3}\|\vec{h}(0)\|_{\mathcal{H}(r \geq \eta)}+R^{-11 / 6}\|\vec{h}(0)\|_{\mathcal{H}(r \geq \eta)}^{2}+\|\vec{h}(0)\|_{\mathcal{H}(r \geq \eta)}^{3}
$$

Proof. The small data global well-posedness and spacetime estimate (6.22) follow from standard contraction mapping and continuity arguments using Strichartz estimates for free waves on $\mathbb{R} \times \mathbb{R}_{*}^{5}$ with Dirichlet boundary condition (see [14]). We now prove (6.23). By the Duhamel formula and Strichartz estimates we have

$$
\begin{aligned}
\sup _{t \in \mathbb{R}}\left\|\vec{h}(t)-\vec{h}_{L}(t)\right\|_{\mathcal{H}(r \geq \eta)} & \lesssim\left\|N_{R}(\cdot, \cdot, h)\right\|_{L_{t}^{1} L_{x}^{2}\left(\mathbb{R} \times \mathbb{R}_{*}^{5}\right)} \\
& \lesssim\left\|V_{e, R} h\right\|_{L_{t}^{1} L_{x}^{2}\left(\mathbb{R} \times \mathbb{R}_{*}^{5}\right)}+\left\|F_{e, R}(\cdot, \cdot, h)\right\|_{L_{t}^{1} L_{x}^{2}\left(\mathbb{R} \times \mathbb{R}_{*}^{5}\right)} \\
& +\left\|G_{e}(\cdot, h)\right\|_{L_{t}^{1} L_{x}^{2}\left(\mathbb{R} \times \mathbb{R}_{*}^{5}\right)}
\end{aligned}
$$

The third term is readily estimated by (6.22) and (6.23)

$$
\left\|G_{e}(\cdot, h)\right\|_{L_{t}^{1} L_{x}^{2}\left(\mathbb{R} \times \mathbb{R}_{*}^{5}\right)} \lesssim\left\|h^{3}\right\|_{L_{t}^{1} L_{x}^{2}\left(\mathbb{R} \times \mathbb{R}_{*}^{5}\right)} \lesssim\|\vec{h}(0)\|_{\mathcal{H}(r \geq \eta)}^{3} .
$$

For the first term we have

By (6.19)

$$
\left\|V_{e, R} h\right\|_{L_{t}^{1} L_{x}^{2}\left(\mathbb{R} \times \mathbb{R}_{*}^{5}\right)} \leq\left\|V_{e, R}\right\|_{L_{t}^{3 / 2} L_{x}^{3}\left(\mathbb{R} \times \mathbb{R}_{*}^{5}\right)}\|h\|_{L_{t}^{3} L_{x}^{6}\left(\mathbb{R} \times \mathbb{R}_{*}^{5}\right)} \lesssim\left\|V_{e, R}\right\|_{L_{t}^{3 / 2} L_{x}^{3}\left(\mathbb{R} \times \mathbb{R}_{*}^{5}\right)}\|\vec{h}(0)\|_{\mathcal{H}(r \geq \eta)} .
$$

$$
\left\|V_{e, R}\right\|_{L_{x}^{3}\left(\mathbb{R}_{*}^{5}\right)}^{3} \lesssim \int_{0}^{R+|t|}(R+|t|)^{-12} r^{4} d r+\int_{R+|t|}^{\infty} r^{-12} r^{4} d r \lesssim(R+|t|)^{-7}
$$


Hence,

$$
\left\|V_{e, R}\right\|_{L_{t}^{3 / 2} L_{x}^{3}\left(\mathbb{R} \times \mathbb{R}_{*}^{5}\right)}^{3 / 2} \lesssim(R+|t|)^{-7 / 2} d t \lesssim R^{-5 / 2}
$$

Thus,

$$
\left\|V_{e, R} h\right\|_{L_{t}^{1} L_{x}^{2}\left(\mathbb{R} \times \mathbb{R}_{*}^{5}\right)} \lesssim R^{-5 / 3}\|\vec{h}(0)\|_{\mathcal{H}(r \geq 1)} .
$$

Similarly, using (6.20) and (6.22) we conclude that $\left\|F_{e, R}(\cdot, \cdot, h)\right\|_{L_{t}^{1} L_{x}^{2}\left(\mathbb{R} \times \mathbb{R}_{*}^{5}\right)} \lesssim R^{-11 / 6}\|h(0)\|_{\mathcal{H}(r \geq 1)}^{2}$ which proves (6.23).

Proof of Lemma 6.6 We first prove Lemma 6.6 for $t=0$. For $R>\eta$, define the truncated initial data $\vec{u}_{R}(0)=\left(u_{0, R}, u_{1, R}\right) \in \mathcal{H}_{0}(r \geq \eta)$ via

$$
\begin{aligned}
& u_{0, R}= \begin{cases}u_{e}(0, r) & \text { if } r \geq R, \\
\frac{r-\eta}{R-\eta} u_{e}(0, R) & \text { if } r<R,\end{cases} \\
& u_{1, R}= \begin{cases}\partial_{t} u_{e}(0, r) & \text { if } r \geq R, \\
0 & \text { if } r<R .\end{cases}
\end{aligned}
$$

Note that for $R$ large,

$$
\left\|\vec{u}_{R}(0)\right\|_{\mathcal{H}(r \geq \eta)} \lesssim\left\|\vec{u}_{e}(0)\right\|_{\mathcal{H}(r \geq R)}
$$

In particular, by Lemma 6.2, there exists $R_{0}>\eta$ such that for all $R \geq R_{0},\left\|\vec{u}_{R}(0)\right\| \leq \delta_{0}$ where $\delta_{0}$ is from Lemma 6.8. Let $u_{R}(t)$ be the solution to (6.18) with initial data $\left(u_{0, R}, u_{1, R}\right)$, and let $\vec{u}_{R, L}(t) \in \mathcal{H}_{0}(r \geq \eta)$ be the solution to the free wave equation $\partial_{t}^{2} u_{R, L}-\Delta u_{R, L}=0,(t, x) \in \mathbb{R} \times \mathbb{R}_{*}^{5}, \vec{u}_{R, L}(0)=\left(u_{0, R}, u_{1, R}\right)$. By finite speed of propagation

$$
r \geq R+|t| \Longrightarrow \vec{u}_{R}(t, r)=\vec{u}_{e}(t, r) \text {. }
$$

By Proposition 6.5, for all $t \geq 0$ or for all $t \leq 0$,

$$
\left\|\pi_{R}^{\perp} \vec{u}_{R, L}(0)\right\|_{\mathcal{H}(r \geq R)} \lesssim\left\|\vec{u}_{R, L}(t)\right\|_{\mathcal{H}(r \geq R+|t|)} .
$$

Suppose, without loss of generality, that the above bound holds for all $t \geq 0$. By (6.23) we conclude that for all $t \geq 0$

$$
\begin{aligned}
\left\|\vec{u}_{e}(t)\right\|_{\mathcal{H}(r \geq R+|t|)} & \geq\left\|\vec{u}_{R, L}(t)\right\|_{\mathcal{H}(r \geq R+|t|)}-\left\|\vec{u}_{R}(t)-\vec{u}_{R, L}(t)\right\|_{\mathcal{H}(r \geq \eta)} \\
& \geq c\left\|\pi_{R}^{\perp} \vec{u}_{R, L}(0)\right\|_{\mathcal{H}(r \geq R)}-C\left[R^{-5 / 3}\left\|u_{R}(0)\right\|_{\mathcal{H}(r \geq \eta)}+R^{-11 / 6}\left\|\vec{u}_{R}(0)\right\|_{\mathcal{H}(r \geq \eta)}^{2}+\left\|\vec{u}_{R}(0)\right\|_{\mathcal{H}(r \geq \eta)}^{3}\right] .
\end{aligned}
$$

Letting $t \rightarrow \infty$ and using the decay property (6.1) and the definition of $\left(u_{0, R}, u_{1, R}\right)$, we conclude that

$$
\left\|\pi_{R}^{\perp} \vec{u}_{e}(0)\right\|_{\mathcal{H}(r \geq R)} \lesssim R^{-5 / 3}\left\|u_{e}(0)\right\|_{\mathcal{H}(r \geq R)}+R^{-11 / 6}\left\|\vec{u}_{e}(0)\right\|_{\mathcal{H}(r \geq R)}^{2}+\left\|\vec{u}_{e}(0)\right\|_{\mathcal{H}(r \geq R)}^{3} .
$$

Note that $\left\|\vec{u}_{e}(0)\right\|_{\mathcal{H}(r \geq R)}^{2}=\left\|\pi_{R}^{\perp} \vec{u}_{e}(0)\right\|_{\mathcal{H}(r \geq R)}^{2}+\left\|\pi_{R} \vec{u}_{e}(0)\right\|_{\mathcal{H}(r \geq R)}^{2}$. Thus, if we take $R_{0}$ large enough to absorb terms involving $\left\|\pi_{R}^{\perp} \vec{u}_{e}(0)\right\|_{\mathcal{H}(r \geq R)}$ into the left hand side in the previous estimate, we obtain for all $R \geq R_{0}$

$$
\left\|\pi_{R}^{\perp} \vec{u}_{e}(0)\right\|_{\mathcal{H}(r \geq R)} \lesssim R^{-5 / 3}\left\|\pi_{R} u_{e}(0)\right\|_{\mathcal{H}(r \geq R)}+R^{-11 / 6}\left\|\pi_{R} \vec{u}_{e}(0)\right\|_{\mathcal{H}(r \geq R)}^{2}+\left\|\pi_{R} \vec{u}_{e}(0)\right\|_{\mathcal{H}(r \geq R)^{\prime}}^{3}
$$

as desired. This proves Lemma 6.6 for $t=0$.

For general $t=t_{0}$ in (6.18), we first set

$$
\begin{aligned}
& u_{0, R, t_{0}}= \begin{cases}u_{e}\left(t_{0}, r\right) & \text { if } r \geq R, \\
\frac{r-\eta}{R-\eta} u\left(t_{0}, R\right) & \text { if } r<R,\end{cases} \\
& u_{1, R, t_{0}}= \begin{cases}\partial_{t} u_{e}\left(t_{0}, r\right) & \text { if } r \geq R, \\
0 & \text { if } r<R .\end{cases}
\end{aligned}
$$

By (6.7) we can find $R_{0}=R_{0}\left(\delta_{0}, \eta\right)$ independent of $t_{0}$ such that for all $R \geq R_{0}$

$$
\left\|\left(u_{0, R, t_{0}}, u_{1, R, t_{0}}\right)\right\|_{\mathcal{H}(r \geq \eta)} \lesssim\left\|\vec{u}_{e}\left(t_{0}\right)\right\|_{\mathcal{H}(r \geq R)} \leq \delta_{0}
$$

The previous argument for $t_{0}=0$ repeated with obvious modifications yield (6.17) for $t=t_{0}$. 
Before proceeding to the next step, it will be useful to reformulate the conclusion of Lemma 6.6 Define

$$
\begin{aligned}
& \lambda(t, r):=r^{3} u_{e}(t, r), \\
& \mu(t, r):=r \int_{r}^{\infty} \partial_{t} u_{e}(t, \rho) \rho d \rho .
\end{aligned}
$$

We denote $\lambda(r):=\lambda(0, r)$ and $\mu(r):=\mu(0, r)$. By (6.15) and (6.16) the functions $\lambda$ and $\mu$ arise in the explicit computation of $\pi_{R}^{\perp} \vec{u}(t)$ and $\pi_{R} \vec{u}(t)$ as follows:

$$
\begin{aligned}
\left\|\pi_{R}^{\perp} \vec{u}_{e}(t)\right\|_{\mathcal{H}(r \geq R)}^{2} & =\int_{R}^{\infty}\left(\frac{1}{r} \partial_{r} \lambda(t, r)\right)^{2} d r+\int_{R}^{\infty}\left(\partial_{r} \mu(t, r)\right)^{2} d r, \\
\left\|\pi_{R} \vec{u}_{e}(t)\right\|_{\mathcal{H}(r \geq R)}^{2} & =3 R^{-3} \lambda^{2}(t, R)+R^{-1} \mu^{2}(t, R) .
\end{aligned}
$$

Thus, Lemma 6.6 can be restated using $\lambda, \mu$ in the following way.

Lemma 6.9. Let $\mu, \lambda$ be as in (6.27) and (6.28). Then there exists $R_{0} \geq \eta$ such that for all $R>R_{0}$ and for all $t \in \mathbb{R}$

$$
\begin{aligned}
\int_{R}^{\infty}\left(\frac{1}{r} \partial_{r} \lambda(t, r)\right)^{2} d r+\int_{R}^{\infty}\left(\partial_{r} \mu(t, r)\right)^{2} d r & \lesssim R^{-19 / 3} \lambda^{2}(t, R)+R^{-29 / 3} \lambda^{4}(t, R)+R^{-9} \lambda^{6}(t, R) \\
& +R^{-13 / 3} \mu^{2}(t, R)+R^{-17 / 3} \mu^{4}(t, R)+R^{-3} \mu^{6}(t, R) .
\end{aligned}
$$

6.1.2. Step 2: Asymptotics for $\vec{u}_{e}(0)$. In this step, we prove that $\vec{u}_{e}(0)$ has the asymptotic expansions (6.11), (6.12) which we now formulate as a lemma.

Lemma 6.10. Let $u_{e}$ be a solution to (6.2) which satisfies (6.7). Let $\vec{u}_{e}(0)=\left(u_{e, 0}, u_{e, 1}\right)$. Then there exists $\alpha \in \mathbb{R}$ such that

$$
\begin{aligned}
r^{3} u_{e, 0}(r) & =\alpha+O\left(r^{-1}\right), \\
r \int_{r}^{\infty} u_{e, 1}(\rho) \rho d \rho & =O\left(r^{-1}\right),
\end{aligned}
$$

as $r \rightarrow \infty$. Equivalently, with $\lambda$ and $\mu$ defined as in (6.27) and (6.28), there exists $\alpha \in \mathbb{R}$ such that

$$
\begin{aligned}
& \lambda(r)=\alpha+O\left(r^{-1}\right), \\
& \mu(r)=O\left(r^{-1}\right) .
\end{aligned}
$$

The proof of Lemma 6.10 is split up into a few further lemmas. First, we use Lemma 6.9 to prove the following difference estimate for $\lambda$ and $\mu$.

Lemma 6.11. Let $\delta_{1} \leq \delta_{0}$ where $\delta_{0}$ is from Lemma 6.8 Let $R_{1} \geq R_{0}>1$ be large enough so that for all $R \geq R_{1}$ and for all $t \in \mathbb{R}$

$$
\begin{aligned}
\left\|\vec{u}_{e}(t)\right\|_{\mathcal{H}(r \geq R)} & \leq \delta_{1}, \\
R^{-5 / 3} & \leq \delta_{1} .
\end{aligned}
$$

Then for all $r, r^{\prime}$ with $R_{1} \leq r \leq r^{\prime} \leq 2 r$ and for all $t \in \mathbb{R}$

$$
\begin{aligned}
\left|\lambda(t, r)-\lambda\left(t, r^{\prime}\right)\right| & \lesssim r^{-5 / 3}|\lambda(t, r)|+r^{-10 / 3}|\lambda(t, r)|^{2}+r^{-3}|\lambda(t, r)|^{3} \\
& +r^{-2 / 3}|\mu(t, r)|+r^{-4 / 3}|\mu(t, r)|^{2}+|\mu(t, r)|^{3}, \\
\left|\mu(t, r)-\mu\left(t, r^{\prime}\right)\right| & \lesssim r^{-8 / 3}|\lambda(t, r)|+r^{-13 / 3}|\lambda(t, r)|^{2}+r^{-4}|\lambda(t, r)|^{3} \\
& +r^{-5 / 3}|\mu(t, r)|+r^{-7 / 3}|\mu(t, r)|^{2}+r^{-1}|\mu(t, r)|^{3} .
\end{aligned}
$$


Proof. By the fundamental theorem of calculus and Lemma 6.9 we have, for $r, r^{\prime}$ such that $R_{1} \leq r \leq r^{\prime} \leq 2 r$,

$$
\begin{aligned}
\left|\lambda(t, r)-\lambda\left(t, r^{\prime}\right)\right|^{2} & =\left(\int_{r}^{r^{\prime}} \partial_{\rho} \lambda(t, \rho) d \rho\right)^{2} \\
& \leq\left(\int_{r}^{r^{\prime}} \rho^{2} d \rho\right)\left(\int_{r}^{r^{\prime}}\left(\frac{1}{\rho} \partial_{\rho} \lambda(t, \rho)\right)^{2} d \rho\right) \\
& \lesssim r^{3}\left(r^{-19 / 3} \lambda^{2}(t, r)+r^{-29 / 3} \lambda^{4}(t, r)+r^{-9} \lambda^{6}(t, r)\right. \\
& \left.+r^{-13 / 3} \mu^{2}(t, r)+r^{-17 / 3} \mu^{4}(t, r)+r^{-3} \mu^{6}(t, r)\right)
\end{aligned}
$$

which proves (6.33).

Similarly, we have

$$
\begin{aligned}
\left|\mu(t, r)-\mu\left(t, r^{\prime}\right)\right|^{2} & \leq r\left(\int_{r}^{r^{\prime}}(\mu(t, \rho))^{2} d \rho\right) \\
& \lesssim r\left(r^{-19 / 3} \lambda^{2}(t, r)+r^{-29 / 3} \lambda^{4}(t, r)+r^{-9} \lambda^{6}(t, r)\right. \\
& \left.+r^{-13 / 3} \mu^{2}(t, r)+r^{-17 / 3} \mu^{4}(t, r)+r^{-3} \mu^{6}(t, r)\right)
\end{aligned}
$$

which proves (6.34).

A simple consequence of Lemma6.11 is the following.

Corollary 6.12. Let $R_{1}$ be as in Lemma 6.11 Then for all $r, r^{\prime}$ with $R_{1} \leq r \leq r^{\prime} \leq 2 r$ and for all $t \in \mathbb{R}$

$$
\begin{aligned}
& \left|\lambda(t, r)-\lambda\left(t, r^{\prime}\right)\right| \lesssim \delta_{1}|\lambda(t, r)|+r \delta_{1}|\mu(t, r)|, \\
& \left|\mu(t, r)-\mu\left(t, r^{\prime}\right)\right| \lesssim r^{-1} \delta_{1}|\lambda(t, r)|+\delta_{1}|\mu(t, r)| .
\end{aligned}
$$

Next we establish the following improved growth rate for $\lambda$ and $\mu$.

Lemma 6.13. For all $t \in \mathbb{R}$,

$$
\begin{aligned}
& |\lambda(t, r)| \lesssim r^{1 / 6}, \\
& |\mu(t, r)| \lesssim r^{1 / 18} .
\end{aligned}
$$

Proof. As in the proof of Lemma 6.6 we only consider the case $t=0$. Fix $r_{0} \geq R_{1}$. By Corollary 6.12

$$
\begin{aligned}
& \left|\lambda\left(2^{n+1} r\right)\right| \leq\left(1+C_{1} \delta_{1}\right)\left|\lambda\left(2^{n} r_{0}\right)\right|+\left(2^{n} r_{0}\right) C_{1} \delta_{1}\left|\mu\left(2^{n} r_{0}\right)\right|, \\
& \left|\mu\left(2^{n+1} r\right)\right| \leq\left(1+C_{1} \delta_{1}\right)\left|\mu\left(2^{n} r_{0}\right)\right|+\left(2^{n} r_{0}\right)^{-1} C_{1} \delta_{1}\left|\lambda\left(2^{n} r_{0}\right)\right| .
\end{aligned}
$$

If we define $a_{n}:=\left|\mu\left(2^{n} r_{0}\right)\right|$ and $b_{n}:=\left(2^{n} r_{0}\right)^{-1}\left|\lambda\left(2^{n} r_{0}\right)\right|$, then (6.39) and (6.40) imply

$$
a_{n+1}+b_{n+1} \leq\left(1+2 C_{1} \delta_{1}\right)\left(a_{n}+b_{n}\right) \text {. }
$$

By induction

$$
a_{n}+b_{n} \leq\left(1+2 C_{1} \delta_{1}\right)^{n}\left(a_{0}+b_{0}\right) .
$$

Choose $\delta_{1}$ so small so that $1+2 C_{1} \delta_{1}<2^{1 / 18}$. We conclude that

$$
a_{n} \leq C\left(2^{n} r_{0}\right)^{1 / 18}
$$

where $C=C\left(r_{0}\right)$. This proves (6.38) for $r=2^{n} r_{0}$. Define

$$
c_{n}=\left|\lambda\left(2^{n} r_{0}\right)\right|=\left(2^{n} r_{0}\right) b_{n} .
$$

Using (6.41) and (6.33) we have, for some $C=C\left(r_{0}\right)$,

$$
c_{n+1} \leq\left(1+C_{1} \delta_{1}\right) c_{n}+C \delta_{1}\left(2^{n} r_{0}\right)^{1 / 6} .
$$


By induction

$$
\begin{aligned}
c_{n} & \leq\left(1+C_{1} \delta_{1}\right)^{n} c_{0}+C r_{0}^{1 / 6} \sum_{k=1}^{n}\left(1+C_{1} \delta_{1}\right)^{n-k} 2^{(k-1) / 6} \\
& \leq C\left(2^{n} r_{0}\right)^{1 / 6} .
\end{aligned}
$$

This proves (6.37) for $r=2^{n} r_{0}$.

To establish (6.37) and (6.38) for general $r$, let $r \geq r_{0}$ such that for some $n \geq 0,2^{n} r_{0} \leq r \leq 2^{n+1} r_{0}$. Then applying (6.33) to the pair $2^{n} r_{0}, r$, we conclude that

$$
\begin{aligned}
|\lambda(r)| & \leq\left|\lambda\left(2^{n} r_{0}\right)\right|+\left|\lambda\left(2^{n} r_{0}\right)-\lambda(r)\right| \\
& \leq C\left(2^{n} r_{0}\right)^{1 / 6}+\left[\left(2^{n} r_{0}\right)^{-5 / 3}\left(2^{n} r_{0}\right)^{1 / 6}+\left(2^{n} r_{0}\right)^{-10 / 3}\left(2^{n} r_{0}\right)^{1 / 3}+\left(2^{n} r_{0}\right)^{-3}\left(2^{n} r_{0}\right)^{1 / 2}\right] \\
& \leq C\left(2^{n} r_{0}\right)^{1 / 6} \\
& \leq C r^{1 / 6},
\end{aligned}
$$

where $C=C\left(r_{0}\right)$. This proves (6.37). A similar argument also establishes the bound (6.38) for all $r \geq r_{0}$ as well.

We now show that for each $t \in \mathbb{R}, \mu(t, r)$ has a limit $\beta(t)$ as $r \rightarrow \infty$.

Lemma 6.14. For all $t \in \mathbb{R}$, there exists $\beta(t) \in \mathbb{R}$ such that

$$
|\mu(t, r)-\beta(t)| \leq C r^{-1} .
$$

The constant $C>0$ is uniform in time.

Proof. We only consider the case $t=0$. The general case follows, again, by using the decay of the trajectory $\vec{u}_{e}(t)$ on exterior regions. Let $R_{1}>1$ be as in Lemma 6.11, and fix $r_{0} \geq R_{1}$. Then Lemma 6.13 and (6.34) yield the estimate

$$
\begin{aligned}
\left|\mu\left(2^{n+1} r_{0}\right)-\mu\left(2^{n} r_{0}\right)\right| & \lesssim\left(2^{n} r_{0}\right)^{-8 / 3}\left(2^{n} r_{0}\right)^{1 / 6}+\left(2^{n} r_{0}\right)^{-13 / 3}\left(2^{n} r_{0}\right)^{1 / 3}+\left(2^{n} r_{0}\right)^{-4}\left(2^{n} r_{0}\right)^{1 / 2} \\
& +\left(2^{n} r_{0}\right)^{-5 / 3}\left(2^{n} r_{0}\right)^{1 / 18}+\left(2^{n} r_{0}\right)^{-7 / 3}\left(2^{n} r_{0}\right)^{1 / 9}+\left(2^{n} r_{0}\right)^{-1}\left(2^{n} r_{0}\right)^{1 / 6} \\
& \lesssim\left(2^{n} r_{0}\right)^{-5 / 6} \\
& \lesssim 2^{-5 n / 6},
\end{aligned}
$$

where the implied constant is uniform in $r_{0}$. Thus,

$$
\sum_{n \geq 0}\left|\mu\left(2^{n+1} r_{0}\right)-\mu\left(2^{n} r_{0}\right)\right| \lesssim 1
$$

with a constant uniform in $r_{0}$. This implies that there exists $\beta=\beta \in \mathbb{R}$ such that $\lim _{n \rightarrow \infty} \mu\left(2^{n} r_{0}\right)=\beta$. Moreover, the sequence $\left\{\mu\left(2^{n} r_{0}\right)\right\}_{n}$ is bounded by a constant depending only on $r_{0}$ since

$$
\begin{aligned}
\left|\mu\left(2^{n} r_{0}\right)\right| & \leq\left|\mu\left(r_{0}\right)\right|+\left|\mu\left(2^{n} r_{0}\right)-\mu\left(r_{0}\right)\right| \\
& =\left|\mu\left(r_{0}\right)\right|+\left|\sum_{k=0}^{n-1}\left(\mu\left(2^{k+1} r_{0}\right)-\mu\left(2^{k} r_{0}\right)\right)\right| \\
& \leq\left|\mu\left(r_{0}\right)\right|+C_{1} \sum_{n \geq 0} 2^{-5 n / 6} \leq C\left(r_{0}\right) .
\end{aligned}
$$

Inserting this bound into the difference estimate (6.34) improves the previous bound on $\left|\mu\left(2^{n+1} r_{0}\right)-\mu\left(2^{n} r_{0}\right)\right|$ to

$$
\left|\mu\left(2^{n+1} r_{0}\right)-\mu\left(2^{n} r_{0}\right)\right| \leq C\left(2^{n} r_{0}\right)^{-1},
$$


where $C=C\left(r_{0}\right)$. Now let $r \geq r_{0}$ such that $2^{n} r_{0} \leq r \leq 2^{n+1} r_{0}$. By Lemma 6.13, (6.34), and our improved bound (6.43), we have that

$$
\begin{aligned}
|\mu(r)-\beta| & \leq\left|\mu(r)-\mu\left(2^{n} r_{0}\right)\right|+\left|\beta-\mu\left(2^{n} r_{0}\right)\right| \\
& =\left|\mu(r)-\mu\left(2^{n} r_{0}\right)\right|+\left|\sum_{k \geq n}\left(\mu\left(2^{k+1} r_{0}\right)-\mu\left(2^{k} r_{0}\right)\right)\right| \\
& \lesssim\left(2^{n} r_{0}\right)^{-1}+\sum_{k \geq n}\left(2^{k} r_{0}\right)^{-1} \\
& \lesssim\left(2^{n} r_{0}\right)^{-1} \\
& \lesssim r^{-1} .
\end{aligned}
$$

This proves (6.42).

We now conclude the proof of the bound (6.32) in Proposition 6.10

Lemma 6.15. Let $\beta(t)$ be as in Lemma6.14 Then $\beta(t) \equiv 0$.

Proof. The proof follows in two steps.

Step 1. We first show that $\beta(t)$ is constant in time. By Lemma 6.14 and the definition of $\mu$, we have shown that

$$
\beta(t)=r \int_{r}^{\infty} \partial_{t} u_{e}(t, \rho) \rho d \rho+O\left(r^{-1}\right),
$$

where the $O\left(r^{-1}\right)$ is uniform in time. Fix $t_{1}<t_{2}$. Since $u_{e}$ solves (6.2), we have for $R \geq R_{1}$,

$$
\begin{aligned}
\beta\left(t_{2}\right)-\beta\left(t_{1}\right) & =\frac{1}{R} \int_{R}^{2 R} \beta\left(t_{2}\right)-\beta\left(t_{1}\right) d s \\
& =\frac{1}{R} \int_{R}^{2 R} s \int_{s}^{\infty}\left[\partial_{t} u_{e}\left(t_{2}, r\right)-\partial_{t} u_{e}\left(t_{1}, r\right)\right] r d r d s+O\left(R^{-1}\right) \\
& =\frac{1}{R} \int_{R}^{2 R} s \int_{s}^{\infty} \int_{t_{1}}^{t_{2}} \partial_{t}^{2} u_{e}(t, r) d t r d r d s+O\left(R^{-1}\right) \\
& =\frac{1}{R} \int_{R}^{2 R} s \int_{s}^{\infty} \int_{t_{1}}^{t_{2}} r^{-3} \partial_{r}\left(r^{4} \partial_{r} u_{e}(t, r)\right) d t d r d s \\
& +\frac{1}{R} \int_{R}^{2 R} s \int_{s}^{\infty} \int_{t_{1}}^{t_{2}}\left[-r V_{e}(r) u_{e}(t, r)+r N_{e}\left(r, u_{e}(t, r)\right)\right] d t d r d s+O\left(R^{-1}\right) \\
& =: A+B+O\left(R^{-1}\right) .
\end{aligned}
$$

We first estimate $B$. Recall that $\lambda(t, r)=r^{3} u_{e}(t, r)$. By (6.37),

$$
\left|u_{e}(t, r)\right| \lesssim r^{-17 / 6},
$$

uniformly in $t$. This estimate, (6.8), (6.9), and (6.10), imply that

$$
\begin{aligned}
|B| & \lesssim\left(t_{2}-t_{1}\right) \frac{1}{R} \int_{R}^{2 R} s \int_{s}^{\infty}\left[r^{-35 / 6}+r^{-23 / 3}+r^{-15 / 2}\right] d r d s \\
& \lesssim\left(t_{2}-t_{1}\right) \frac{1}{R} \int_{R}^{2 R} s \int_{s}^{\infty} r^{-5} d r d s \\
& \lesssim\left(t_{2}-t_{1}\right) R^{-3}
\end{aligned}
$$


For $A$, we repeatedly use integration by parts and use (6.44) to conclude that

$$
\begin{aligned}
A & =\frac{3}{R} \int_{t_{1}}^{t_{2}} \int_{R}^{2 R} s \int_{s}^{\infty} \partial_{r} u_{e}(t, r) d r d s d t-\frac{1}{R} \int_{t_{1}}^{t_{2}} \int_{R}^{2 R} s^{2} \partial_{s} u_{e}(t, s) d s d t \\
& =-\frac{3}{R} \int_{t_{1}}^{t_{2}} \int_{R}^{2 R} s \partial_{s} u_{e}(t, s) d s d t-\frac{1}{R} \int_{t_{1}}^{t_{2}} \int_{R}^{2 R} s^{2} \partial_{s} u_{e}(t, s) d s d t \\
& =-\frac{1}{R} \int_{t_{1}}^{t_{2}} \int_{R}^{2 R} s \partial_{s} u_{e}(t, s) d s d t+\int_{t_{1}}^{t_{2}}\left[R u_{e}(t, R)-2 R u_{e}(t, 2 R)\right] d t \\
& =O\left(t_{2}-t_{1}\right) O\left(R^{-11 / 6}\right) .
\end{aligned}
$$

In summary, we have that $|A|+|B| \lesssim R^{-1}\left(t_{2}-t_{1}\right)$ so that

$$
\beta\left(t_{2}\right)-\beta\left(t_{1}\right)=O\left(t_{2}-t_{1}\right) O\left(R^{-1}\right)+O\left(R^{-1}\right) .
$$

Letting $R \rightarrow \infty$ implies that $\beta\left(t_{2}\right)=\beta\left(t_{1}\right)$ as desired. This completed Step 1 .

Step 2. By Step 1, we have that $\beta(t)=\beta(0)=: \beta$ for all $t \in \mathbb{R}$. In this step, we show that $\beta=0$ which concludes the proof of Lemma6.15 By Lemma 6.14 and Step 1, for all $R \geq R_{1}$ and for all $t \in \mathbb{R}$ we have

$$
\beta=R \int_{R}^{\infty} \partial_{t} u_{e}(t, r) r d r+O\left(R^{-1}\right),
$$

where the $O\left(R^{-1}\right)$ term is uniform in time. Integrating the previous expression from 0 to $T$, dividing by $T$, and using (6.44) yield for all $T>0$ and $R \geq R_{1}$

$$
\begin{aligned}
\beta & =\frac{R}{T} \int_{R}^{\infty} \int_{0}^{T} \partial_{t} u_{e}(t, r) d t r d r+O\left(R^{-1}\right) \\
& =\frac{R}{T} \int_{R}^{\infty}\left[u_{e}(T, r)-u_{e}(0, r)\right] r d r+O\left(R^{-1}\right) \\
& =O\left(\frac{R^{1 / 6}}{T}\right)+O\left(R^{-1}\right) .
\end{aligned}
$$

If we now choose $R=T$ and let $T \rightarrow \infty$, we conclude that $\beta=0$ as desired. This concludes Step 2 and the proof of Lemma 6.15 .

Lemma 6.16. There exists $\alpha \in \mathbb{R}$ such that

$$
|\lambda(r)-\alpha| \lesssim r^{-1}
$$

Proof. The proof of Lemma 6.16 is very similar to the proof for Lemma 6.14 and we only sketch it. Fix $r_{0} \geq R_{1}$. By Lemma 6.15, the difference estimate 6.33, and the growth estimate 6.37 we have

$$
\begin{aligned}
\left|\lambda\left(2^{n+1} r_{0}\right)-v_{0}\left(2^{n} r_{0}\right)\right| & \lesssim\left(2^{n} r_{0}\right)^{-5 / 3}\left(2^{n} r_{0}\right)^{1 / 6}+\left(2^{n} r_{0}\right)^{-10 / 3}\left(2^{n} r_{0}\right)^{1 / 3}+\left(2^{n} r_{0}\right)^{-3}\left(2^{n} r_{0}\right)^{1 / 2} \\
& +\left(2^{n} r_{0}\right)^{-2 / 3}\left(2^{n} r_{0}\right)^{-1}+\left(2^{n} r_{0}\right)^{-4 / 3}\left(2^{n} r_{0}\right)^{-2}+\left(2^{n} r_{0}\right)^{-3} \\
& \lesssim\left(2^{n} r_{0}\right)^{-3 / 2} .
\end{aligned}
$$

Thus,

$$
\sum_{n \geq 0}\left|\lambda\left(2^{n+1} r_{0}\right)-\lambda\left(2^{n} r_{0}\right)\right|<\infty
$$

so that there exists $\alpha \in \mathbb{R}$ such that $\lim _{n} \lambda\left(2^{n} r_{0}\right)=\alpha$. As in the proof of Lemma 6.14, we then use the fact that the sequence $\left\{\lambda\left(2^{n} r_{0}\right)\right\}_{n}$ is bounded and the difference estimate 6.37 to conclude that for $r \geq r_{0}$

$$
|\lambda(r)-\alpha| \lesssim r^{-1}
$$

as desired. 
We have shown that there exists $\alpha \in \mathbb{R}$ such that

$$
\begin{aligned}
r^{3} u_{e}(0, r) & =\alpha+O\left(r^{-1}\right), \\
r \int_{r}^{\infty} \partial_{t} u_{e}(0, \rho) \rho d \rho & =O\left(r^{-1}\right),
\end{aligned}
$$

as $r \rightarrow \infty$. In the case $\alpha=0$, we conclude that $\vec{u}(0)=(0,0)$ on $r \geq \eta$.

Lemma 6.17. Let $\alpha$ be as in Lemma 6.16 If $\alpha=0$, then $\vec{u}(0, r)=(0,0)$ for $r \geq \eta$.

Proof. The proof of Lemma 6.17 is split into two steps.

Claim 6.18. Let $\alpha$ be as in Lemma 6.16 If $\alpha=0$, then $\vec{u}(0, r)$ is compactly supported in $r$.

Proof of Claim 6.18 Since $\alpha=0$,

$$
\begin{aligned}
& \lambda(r)=O\left(r^{-1}\right), \\
& \mu(r)=O\left(r^{-1}\right) .
\end{aligned}
$$

Then, for $r_{0} \geq R_{1}$, we have

$$
\left|\lambda\left(2^{n} r_{0}\right)\right|+\left|\mu\left(2^{n} r_{0}\right)\right| \lesssim\left(2^{n} r_{0}\right)^{-1} .
$$

By the difference estimate (6.33) and the growth estimates (6.45), (6.46), we conclude that

$$
\begin{aligned}
& \left|\lambda\left(2^{n+1} r_{0}\right)\right| \geq\left(1-C_{1} \delta_{1}\right)\left|\lambda\left(2^{n} r_{0}\right)\right|-C_{1}\left(2^{n} r_{0}\right)^{-2 / 3}\left|\mu\left(2^{n} r_{0}\right)\right|, \\
& \left|\mu\left(2^{n+1} r_{0}\right)\right| \geq\left(1-C_{1} \delta_{1}\right)\left|\mu\left(2^{n} r_{0}\right)\right|-C_{1}\left(2^{n} r_{0}\right)^{-8 / 3}\left|\lambda\left(2^{n} r_{0}\right)\right| .
\end{aligned}
$$

The constant $C_{1}$ is independent of $\delta_{1}$ and $r_{0}$. Thus

$$
\left|\lambda\left(2^{n+1} r_{0}\right)\right|+\left|\mu\left(2^{n+1} r_{0}\right)\right| \geq\left(1-C_{1} \delta_{1}-C_{1} r_{0}^{-2 / 3}\right)\left[\left|\lambda\left(2^{n} r_{0}\right)\right|+\left|\mu\left(2^{n} r_{0}\right)\right|\right] .
$$

Take $r_{0}$ large and $\delta_{1}$ small enough so that $C_{1}\left(\delta_{1}+r_{0}^{-2 / 3}\right)<1 / 4$. Then

$$
\left|\lambda\left(2^{n+1} r_{0}\right)\right|+\left|\mu\left(2^{n+1} r_{0}\right)\right| \geq \frac{3}{4}\left[\left|\lambda\left(2^{n} r_{0}\right)\right|+\left|\mu\left(2^{n} r_{0}\right)\right|\right] .
$$

Proceeding inductively we obtain

$$
\left|\lambda\left(2^{n+1} r_{0}\right)\right|+\left|\mu\left(2^{n+1} r_{0}\right)\right| \geq\left(\frac{3}{4}\right)^{n}\left[\left|\lambda\left(r_{0}\right)\right|+\left|\mu\left(r_{0}\right)\right|\right] .
$$

By (6.47) we conclude that

$$
\left(\frac{3}{4}\right)^{n}\left[\left|\lambda\left(r_{0}\right)\right|+\left|\mu\left(r_{0}\right)\right|\right] \lesssim\left(2^{n} r_{0}\right)^{-1}
$$

which implies

$$
\left(\frac{3}{2}\right)^{n}\left[\left|\lambda\left(r_{0}\right)\right|+\left|\mu\left(r_{0}\right)\right|\right] \lesssim 1,
$$

where the implied constant is uniform in $n$. Hence $\left(\lambda\left(r_{0}\right), \mu\left(r_{0}\right)\right)=(0,0)$. By $(6.30)\left\|\pi_{r_{0}} \vec{u}_{e}(0)\right\|_{\mathcal{H}\left(r \geq r_{0}\right)}=0$. By Lemma $6.9\left\|\pi_{r_{0}}^{\perp} \vec{u}_{e}(0)\right\|_{\mathcal{H}\left(r \geq r_{0}\right)}=0$. Hence $\left\|\vec{u}_{e}(0)\right\|_{\mathcal{H}\left(r \geq r_{0}\right)}=0$. Since $\lim _{r \rightarrow \infty} u_{e, 0}(r)=0$, we conclude that $\left(u_{e, 0}(r), u_{e, 1}(r)\right)=(0,0)$ for $r \geq r_{0}$. Since $u(t, r)=r^{2}\langle r\rangle^{-2} u_{e}(t, r)$, we conclude that $\vec{u}(0, r)=(0,0)$ on $r \geq r_{0}$ as well. This concludes the proof of the claim.

Claim 6.19. If $\vec{u}(0, r)$ is compactly supported in $(\eta, \infty)$, then $\vec{u}(t, r)=(0,0)$ on $(\eta, \infty)$.

Proof of Claim 6.19 Suppose not, i.e. $\vec{u}(0, r)$ is not identically 0 on $(\eta, \infty)$ Then $\left(u_{e, 0}, u_{e, 1}\right)$ is not identically 0 on $(\eta, \infty)$. Define

$$
\rho_{0}=\inf \left\{\rho:\left\|\vec{u}_{e}(0)\right\|_{\mathcal{H}(r \geq \rho)}=0\right\}
$$

By our assumptions we have that $\eta<\rho<\infty$. Let $\rho_{1}=\rho_{1}\left(\delta_{1}\right)$ be so close to $\rho_{0}$ so that $\eta<\rho_{1}<\rho_{0}$ and

$$
0<\left\|\vec{u}_{e}(0)\right\|_{\mathcal{H}\left(r \geq \rho_{1}\right)}^{2} \leq \delta_{1}^{2},
$$


where $\delta_{1}$ is as in Lemma 6.11

By (6.29) and (6.30) and our choice of $\rho_{1}$, we have that

$$
\begin{aligned}
\int_{\rho_{1}}^{\infty} & \left(\frac{1}{r} \partial_{r} \lambda(r)\right)^{2} d r+\int_{\rho_{1}}^{\infty}\left(\partial_{r} \mu(r)\right)^{2} d r \\
& +3 \rho_{1}^{-3} \lambda^{2}\left(\rho_{1}\right)+\rho_{1}^{-1} \mu^{2}\left(\rho_{1}\right)=\left\|\pi_{\rho_{1}}^{\perp} \vec{u}_{e}(0)\right\|_{\mathcal{H}\left(r \geq \rho_{1}\right)}^{2}+\left\|\pi_{\rho_{1}} \vec{u}_{e}(0)\right\|_{\mathcal{H}\left(r \geq \rho_{1}\right)}^{2}<\delta_{1}^{2} .
\end{aligned}
$$

If we define $\left(u_{0, \rho_{1}}, u_{1, \rho_{1}}\right)$ as in (6.24) and (6.25), we have for $\rho_{1}$ close to $\rho_{0}$,

$$
\left\|\left(u_{0, \rho_{1}}, u_{1, \rho_{1}}\right)\right\|_{\mathcal{H}(r \geq \eta)} \leq C\left(\rho_{0}\right)\left\|\vec{u}_{e}(0)\right\|_{\mathcal{H}\left(r \geq \rho_{1}\right)} \leq \delta_{1} .
$$

Thus, by Lemma 6.9 we obtain

$$
\begin{aligned}
\int_{\rho_{1}}^{\infty}\left(\frac{1}{r} \partial_{r} \lambda(r)\right)^{2} d r+\int_{\rho_{1}}^{\infty}\left(\partial_{r} \mu(r)\right)^{2} d r & \lesssim \rho_{1}^{-19 / 3} \lambda^{2}\left(\rho_{1}\right)+\rho_{1}^{-29 / 3} \lambda^{4}\left(\rho_{1}\right)+R^{-\rho_{1}} \lambda^{6}\left(\rho_{1}\right) \\
& +\rho_{1}^{-13 / 3} \mu^{2}\left(\rho_{1}\right)+\rho_{1}^{-17 / 3} \mu^{4}\left(\rho_{1}\right)+\rho_{1}^{-3} \mu^{6}\left(\rho_{1}\right) \\
& \leq C\left(\rho_{0}\right)\left[\left|\lambda\left(\rho_{1}\right)\right|^{2}+\left|\mu\left(\rho_{1}\right)\right|^{2}\right]
\end{aligned}
$$

as long as $\rho_{1}$ is sufficiently close to $\rho_{0}$. Using the previous estimate and the fact that $\lambda\left(\rho_{0}\right)=0$, we argue as in the proof of Lemma 6.11 to obtain

$$
\begin{aligned}
\left|\lambda\left(\rho_{1}\right)\right|^{2} & =\left|\lambda\left(\rho_{1}\right)-\lambda\left(\rho_{0}\right)\right|^{2} \\
& \leq\left(\rho_{0}-\rho_{1}\right)^{3}\left(\int_{\rho_{1}}^{\rho_{0}}\left(\frac{1}{r} \partial_{r} \lambda(r)\right)^{2} d r\right) \\
& \leq C\left(\rho_{0}\right)\left(\rho_{0}-\rho_{1}\right)^{3}\left[\left|\lambda\left(\rho_{1}\right)\right|^{2}+\left|\mu\left(\rho_{1}\right)\right|^{2}\right] .
\end{aligned}
$$

Similarly,

$$
\left|\mu\left(\rho_{1}\right)\right|^{2} \leq C\left(\rho_{0}\right)\left(\rho_{0}-\rho_{1}\right)\left[\left|\lambda\left(\rho_{1}\right)\right|^{2}+\left|\mu\left(\rho_{1}\right)\right|^{2}\right] .
$$

We conclude that for all $\rho_{1}$ close to $\rho_{0}$,

$$
\left|\lambda\left(\rho_{1}\right)\right|^{2}+\left|\mu\left(\rho_{1}\right)\right|^{2} \leq 2 C\left(\rho_{0}\right)\left(\rho_{0}-\rho_{1}\right)\left[\left|\lambda\left(\rho_{1}\right)\right|^{2}+\left|\mu\left(\rho_{1}\right)\right|^{2}\right]
$$

Thus, $\left(\lambda\left(\rho_{1}\right), \mu\left(\rho_{1}\right)\right)=(0,0)$ for $\rho_{1}<\rho_{0}$ close to $\rho_{0}$. By (6.48) and (6.49) we conclude that $\left\|\vec{u}_{e}(0)\right\|_{\mathcal{H}\left(r \geq \rho_{1}\right)}=0$. This contradicts our definition of $\rho_{0}$ and the fact that $\rho_{1}<\rho_{0}$. Thus, $\rho_{0}=\eta$ and $\left\|\vec{u}_{e}(0)\right\|_{\mathcal{H}(r \geq \eta)}=0$ as desired.

Lemma 6.17now follows immediately from Claim 6.18 and Claim 6.19

Using the previous arguments we can, in fact, conclude more in the case $\alpha=0$.

Lemma 6.20. Let $\alpha$ be as in Lemma 6.16 If $\alpha=0$, then

$$
\vec{u}(t, r)=(0,0)
$$

for all $t \in \mathbb{R}$ and $r>0$.

Proof. By Lemma 6.17 we know that if $\alpha=0$ then $\vec{u}(0, r)=(0,0)$ on $\{r \geq \eta\}$. By finite speed of propagation, we conclude that

$$
\vec{u}(t, r)=(0,0) \quad \text { on }\{r \geq|t|+\eta\} .
$$

Let $t_{0} \in \mathbb{R}$ be arbitrary and define $u_{t_{0}}(t, r)=u\left(t+t_{0}, r\right)$. Then ${\overrightarrow{u_{t}}}_{t_{0}}$ inherits the following compactness property from $\vec{u}$ :

$$
\begin{array}{r}
\forall R \geq 0, \quad \lim _{|t| \rightarrow \infty}\left\|\vec{u}_{t_{0}}(t)\right\|_{\mathcal{H}\left(r \geq R+|t| ;\left(r^{2}+1\right)^{2} d r\right)}=0, \\
\lim _{R \rightarrow \infty}\left[\sup _{t \in \mathbb{R}}\left\|\overrightarrow{\mathcal{u}}_{t_{0}}(t)\right\|_{\mathcal{H}\left(r \geq R+|t| ;\left(r^{2}+1\right)^{2} d r\right)}\right]=0,
\end{array}
$$


and by (6.50) $\vec{u}_{t_{0}}(0, r)$ is supported in $\left\{0<r \leq \eta+\left|t_{0}\right|\right\}$. By Claim 6.19 applied to $\vec{u}_{t_{0}}$ we conclude that $\vec{u}_{t_{0}}(0, r)=(0,0)$ on $r \geq \eta$. Since $t_{0}$ was arbitrary, we conclude that

$$
\vec{u}\left(t_{0}, r\right)=(0,0) \text { on }\{r \geq \eta\},
$$

for any $t_{0} \in \mathbb{R}$. Since $\eta>0$ was arbitrarily fixed in the beginning of this subsection, we conclude that

$$
\vec{u}(t, r)=(0,0)
$$

for all $t \in \mathbb{R}$ and $r>0$ as desired.

6.1.3. Step 3: Conclusion of the proof of Proposition 6.4 We now conclude the proof of Proposition 6.4 by proving the following.

Lemma 6.21. Let $\alpha$ be as in Lemma6.16 As before, we denote the unique finite energy harmonic map of degree $n$ by $Q$ and recall that there exists a unique $\alpha_{n}>0$ such that

$$
Q(r)=n \pi-\alpha_{n} r^{-2}+O\left(r^{-4}\right) .
$$

Let $Q_{\alpha-\alpha_{n}}$ denote the unique solution to (2.1) with the property that

$$
Q_{\alpha-\alpha_{n}}(r)=n \pi+\left(\alpha-\alpha_{n}\right) r^{-2}+O\left(r^{-4}\right)
$$

as $r \rightarrow \infty$. Note that $Q_{\alpha-\alpha_{n}}$ exists and is unique by Proposition 2.4 Define a static solution $U_{+}$to (5.1) via

$$
U_{+}(r)=\langle r\rangle^{-1}\left(Q_{\alpha-\alpha_{n}}(r)-Q(r)\right)
$$

Then

$$
\vec{u}(t, r)=\left(U_{+}(r), 0\right)
$$

for all $t \in \mathbb{R}$ and $r>0$.

Proof. Lemma 6.21 follows from the proof of the $\alpha=0$ case and a change of variables. Let $Q_{\alpha-\alpha_{n}}$ be as in the statement of the lemma. We define

$$
\begin{aligned}
u_{\alpha}(t, r) & :=u(t, r)-\langle r\rangle^{-1}\left(Q_{\alpha-\alpha_{n}}(r)-Q(r)\right) \\
& =u(t, r)-U_{+}(r)
\end{aligned}
$$

and observe that $u_{\alpha}$ solves

$$
\partial_{t}^{2} u_{\alpha}-\partial_{r}^{2} u_{\alpha}-\frac{4 r}{r^{2}+1} \partial_{r} u_{\alpha}+V_{\alpha}(r) u_{\alpha}=N_{\alpha}\left(r, u_{\alpha}\right)
$$

where the potential $V_{\alpha}$ is given by

$$
V_{\alpha}(r)=\langle r\rangle^{-4}+2\langle r\rangle^{-2}\left(\cos 2 Q_{\alpha-\alpha_{n}}-1\right),
$$

and $N_{\alpha}(r, u):=F_{\alpha}(r, u)+G_{\alpha}(r, u)$ with

$$
\begin{aligned}
& F_{\alpha}(r, u):=2\langle r\rangle^{-3} \sin ^{2}(\langle r\rangle u) \sin 2 Q_{\alpha-\alpha_{n} \prime} \\
& G_{\alpha}(r, u):=\langle r\rangle^{-3}[2\langle r\rangle u-\sin (2\langle r\rangle u)] \cos 2 Q_{\alpha-\alpha_{n}} .
\end{aligned}
$$

By (6.51), the potential $V_{\alpha}$ is smooth and satisfies

$$
V_{\alpha}(r)=\langle r\rangle^{-4}+O\left(\langle r\rangle^{-6}\right),
$$

as $r \rightarrow \infty$ and the nonlinearities $F_{\alpha}$ and $G_{\alpha}$ satisfy

$$
\begin{aligned}
& \left|F_{\alpha}(r, u)\right| \lesssim\langle r\rangle^{-3}|u|^{2}, \\
& \left|G_{\alpha}(r, u)\right| \lesssim|u|^{3},
\end{aligned}
$$

for $r \geq 0$. Moreover, by (6.52) we see that $\vec{u}_{\alpha}$ inherits the compactness property from $\vec{u}$ :

$$
\begin{array}{r}
\forall R \geq 0, \quad \lim _{|t| \rightarrow \infty}\left\|\vec{u}_{\alpha}(t)\right\|_{\mathcal{H}\left(r \geq R+|t| ;\left(1+r^{2}\right)^{2} d r\right)}=0, \\
\lim _{R \rightarrow \infty}\left[\sup _{t \in \mathbb{R}}\left\|\vec{u}_{\alpha}(t)\right\|_{\mathcal{H}\left(r \geq R+|t| ;\left(1+r^{2}\right)^{2} d r\right)}\right]=0 .
\end{array}
$$


Let $\eta>0$. We now define for $r \geq \eta$,

$$
u_{\alpha, e}(t, r):=\frac{r^{2}+1}{r^{2}} u_{\alpha}(t, r)
$$

and note that $u_{\alpha, e}$ satisfies an equation analogous to $u_{e}$ :

$$
\partial_{t}^{2} u_{\alpha, e}-\partial_{r}^{2} u_{\alpha, e}-\frac{4}{r} \partial_{r} u_{\alpha, e}+V_{\alpha, e}(r) u_{\alpha, e}=N_{\alpha, e}\left(r, u_{\alpha, e}\right), \quad t \in \mathbb{R}, r \geq \eta,
$$

where

$$
V_{\alpha, e}(r)=V_{\alpha}(r)-\frac{2}{r^{2}\left(r^{2}+1\right)},
$$

and $N_{\alpha, e}\left(r, u_{e}\right)=F_{\alpha, e}\left(r, u_{e}\right)+G_{\alpha, e}\left(r, u_{e}\right)$ where

$$
\begin{aligned}
& F_{\alpha, e}\left(r, u_{\alpha, e}\right)=\frac{r^{2}+1}{r^{2}} F_{\alpha}\left(r, \frac{r^{2}}{r^{2}+1} u_{\alpha, e}\right), \\
& G_{\alpha, e}\left(r, u_{\alpha, e}\right)=\frac{r^{2}+1}{r^{2}} G_{\alpha}\left(r, \frac{r^{2}}{r^{2}+1} u_{\alpha, e}\right) .
\end{aligned}
$$

In particular, we have the analogues of (6.8), (6.9), and (6.10): for all $r \geq \eta$,

$$
\begin{aligned}
\left|V_{\alpha, e}(r)\right| & \lesssim r^{-4}, \\
\left|F_{\alpha, e}(r, u)\right| & \lesssim r^{-3}|u|^{2}, \\
\left|G_{\alpha, e}(r, u)\right| & \lesssim|u|^{3} .
\end{aligned}
$$

Moreover, $u_{\alpha, e}$ inherits the following compactness properties from $u_{\alpha}$ :

$$
\begin{array}{r}
\forall R \geq \eta, \quad \lim _{|t| \rightarrow \infty}\left\|\vec{u}_{\alpha, e}(t)\right\|_{\mathcal{H}\left(r \geq R+|t| ; r^{4} d r\right)}=0, \\
\lim _{R \rightarrow \infty}\left[\sup _{t \in \mathbb{R}}\left\|\vec{u}_{\alpha, e}(t)\right\|_{\mathcal{H}\left(r \geq R+|t| ; r^{4} d r\right)}\right]=0 .
\end{array}
$$

Finally, by construction we see that

$$
\begin{aligned}
r^{3} u_{\alpha, e, 0}(r) & =O\left(r^{-1}\right), \\
r \int_{r}^{\infty} u_{\alpha, e, 1}(\rho) \rho d \rho & =O\left(r^{-1}\right) .
\end{aligned}
$$

Using (6.57)-(6.63), we may repeat the previous arguments with $u_{e, \alpha}$ in place of $u_{e}$ to conclude the following analog of Lemma 6.17

Lemma 6.22. $\vec{u}_{\alpha}(0, r)=(0,0)$ for $r \geq \eta$.

Finally, we obtain the following analog of Lemma 6.20

Lemma 6.23. We have

$$
\vec{u}_{\alpha}(t, r)=(0,0)
$$

for all $t \in \mathbb{R}$ and $r>0$.

Equivalently, Lemma 6.23 states that

$$
\vec{u}(t, r)=\left(U_{+}(r), 0\right)
$$

for all $t \in \mathbb{R}$ and $r>0$. This concludes the proof of Lemma 6.21 and Proposition 6.4 
6.2. Proof of Proposition 6.3. Using Proposition 6.4 and its analog for $r<0$, we quickly conclude the proof of Proposition 6.3 Indeed, we know that there exists static solutions $U_{ \pm}$to (5.1) such that

$$
\vec{u}(t, r)=\left(U_{ \pm}(r), 0\right)
$$

for all $\pm r>0$ and $t \in \mathbb{R}$. In particular, $\partial_{t} u(t, r)=0, \partial_{r} u(t, r)=\partial_{r} u(0, r)$ and $u(t, r)=u(0, r)$ for all $t$ and almost every $r$. Let $\psi \in C_{0}^{\infty}(\mathbb{R})$ with $\int \psi d t=1$ and let $\varphi \in C_{0}^{\infty}(\mathbb{R})$. Then since $u$ solves (5.1) in the weak sense, we conclude that

$$
\begin{aligned}
0 & =\iint\left[\psi^{\prime}(t) \varphi(r) \partial_{t} u(t, r)+\psi(t) \varphi^{\prime}(r) \partial_{r} u(t, r)+V(r) \psi(t) \varphi(r) u(t, r)\right. \\
& -\psi(t) \varphi(r) N(r, u(t, r))]\left(r^{2}+1\right)^{2} d r d t \\
= & \iint \psi(t)\left[\varphi^{\prime}(r) \partial_{r} u(0, r)+V(r) \varphi(r) u(0, r)-\varphi(r) N(r, u(0, r))\right]\left(r^{2}+1\right)^{2} d r d t \\
= & \int\left[\varphi^{\prime}(r) \partial_{r} u(0, r)+V(r) \varphi(r) u(0, r)-\varphi(r) N(r, u(0, r))\right]\left(r^{2}+1\right)^{2} d r .
\end{aligned}
$$

Since $\varphi$ was arbitrary, we see that $u(0, r)$ is a weak solution in $H^{1}(\mathbb{R})$ to the static equation $-\partial_{r}^{2} u-\frac{4 r}{r^{2}+1} \partial_{r} u+$ $V(r) u=N(r, u)$ on $\mathbb{R}$. By standard arguments we conclude that $u(0, r)$ is a classical solution. Thus, $\vec{u}(t, r)=$ $(U(r), 0):=(u(0, r), 0)$ for all $t, r \in \mathbb{R}$ as desired.

6.3. Proofs of Proposition 6.1 and Theorem 4.1. We briefly summarize the proofs of Proposition 6.1 and Theorem 4.1. From Proposition 6.3, we obtain Proposition 6.1

Proof of Proposition 6.1 By Proposition 6.3, we have that $\vec{u}=(U, 0)$ for some finite energy static solution $U$ to (5.1). Thus, $\psi=Q+\langle r\rangle U$ is a finite energy static solution to (4.1), i.e. a harmonic map. By Proposition 2.1. the harmonic map $Q$ is the unique finite energy static solution to (4.1) so that $Q=\psi=Q+\langle r\rangle U$ whence $\vec{u}=(0,0)$ as desired.

Using Proposition 5.3 and Proposition 6.1 we conclude the proof of our main result Theorem 4.1 (equivalently Theorem 1.1).

Proof of Theorem 4.1 Suppose that Theorem 4.1 fails. Then by Proposition 5.3, there exists a nonzero solution $u_{*}$ to (4.4) such that the trajectory

$$
K:=\left\{\vec{u}_{*}(t): t \in \mathbb{R}\right\},
$$

is precompact in $\mathcal{H}$. However, by Proposition 6.1, we must have that $\vec{u}_{*}=(0,0)$, which contradicts the fact that $u_{*}$ is nonzero. Thus, Theorem 4.1 holds.

\section{REFERENCES}

[1] Bahouri, Hajer; Gérard, Patrick. High frequency approximation of solutions to critical nonlinear wave equations. Amer. J. Math. 121 (1999), no. 1, 131-175.

[2] Bizon, Piotr; Chmaj, and M. Maliborski. Equivariant wave maps exterior to a ball. Nonlinearity (5) 25 (2012), 1299-1309.

[3] Bizon, Piotr; Kahl; Michal. Wave maps on a wormhole. Preprint, 122014.

[4] Coulhon, Thierry; Russ, Emmanuel; Tardivel-Nachef, Valérie. Sobolev algebras on Lie groups and Riemannian manifolds. Amer. J. Math. 123 (2001), no. 2, 283-342.

[5] Côte, Raphaël; Kenig, Carlos E.; Schlag, Wilhelm. Energy partition for the linear radial wave equation. Math. Ann. 358 (2014), no. 3-4, 573-607.

[6] Duyckaerts, Thomas; Kenig, Carlos; Merle, Frank. Classification of radial solutions of the focusing, energy critical wave equation. Cambridge Journal Mathematics 1 (2013), no. 1, 74-144.

[7] Franklin, Paul; James, Oliver; Thorne, Kip S.; von Tunzelmann, Eugenie. Visualizing Interstellar's Wormhole. Preprint, 042015.

[8] Geba, Dan-Andrei; Rajeev, Sarada G. A continuity argument for a semilinear Skyrme model. Electron. J. Differential Equations 2010, No. 86, 9 pp.

[9] Geba, Dan-Andrei; Rajeev, S. G. Nonconcentration of energy for a semilinear Skyrme model. Ann. Physics 325 (2010), no. 12, 2697-2706.

[10] Gesztesy, Fritz; Zinchenko, Maxim. On spectral theory for Schrödinger operators with strongly singular potentials. Math. Nachr. 279 (2006), no. 9-10, 1041-1082.

[11] Keel, Markus; Tao, Terence. Endpoint Strichartz estimates. Amer. J. Math. 120 (1998), no. 5, 955-980. 
[12] Kenig, Carlos E.; Merle, Frank. Global well-posedness, scattering and blow-up for the energy-critical, focusing, non-linear Schrödinger equation in the radial case. Invent. Math. 166 (2006), no. 3, 645-675.

[13] Kenig, Carlos E.; Merle, Frank. Global well-posedness, scattering and blow-up for the energy-critical focusing non-linear wave equation. Acta Math. 201 (2008), no. 2, 147-212.

[14] Kenig, Carlos E.; Lawrie, Andrew; Schlag, Wilhelm. Relaxation of wave maps exterior to a ball to harmonic maps for all data. Geom. Funct. Anal. 24 (2014), no. 2, 610-647.

[15] Kenig, Carlos; Lawrie, Andrew; Liu, Baoping; Schlag, Wilhelm. Channels of energy for the linear radial wave equation. Adv. Math. 285 (2015), 877-936.

[16] Kenig, Carlos; Lawrie, Andrew; Liu, Baoping; Schlag, Wilhelm. Stable soliton resolution for exterior wave maps in all equivariance classes. Adv. Math. 285 (2015), 235-300.

[17] Lawrie, Andew; Oh, Sung-Jin; Shahshahani, Sohrab. Equivariant wave maps on the hyperbolic plane with large energy. Preprint, 052015.

[18] Lawrie, Andrew; Schlag, Wilhelm. Scattering for wave maps exterior to a ball. Adv. Math. 232 (2013), 57-97.

[19] McLeod, J. B.; Troy, W. C. The Skyrme model for nucleons under spherical symmetry. Proc. Roy. Soc. Edinburgh Sect. A 118 (1991), no. 3-4, 271-288.

[20] Morris, Michael S.; Thorne, Kip S. Wormholes in spacetime and their use for interstellar travel: a tool for teaching general relativity. Amer. J. Phys. 56 (1988), no. 5, 395-412.

[21] Rodriguez, Casey. Soliton resolution for equivariant wave maps on a wormhole: II. Preprint, 2016.

[22] Schlag, Wilhelm. Semilinear wave equations. ICM Proceedings, 2014.

[23] Schlag, Wilhelm; Soffer, Avy; Staubach, Wolfgang. Decay for the wave and Schrödinger evolutions on manifolds with conical ends. I. Trans. Amer. Math. Soc. 362 (2010), no. 1, 19-52.

[24] Schlag, Wilhelm; Soffer, Avy; Staubach, Wolfgang. Decay for the wave and Schrödinger evolutions on manifolds with conical ends. II. Trans. Amer. Math. Soc. 362 (2010), no. 1, 289-318.

[25] Shatah, Jalal; Struwe, Michael. Geometric wave equations. Courant Lecture Notes in Mathematics, 2. New York University, Courant Institute of Mathematical Sciences, New York; American Mathematical Society, Providence, RI, 1998. viii+153 pp.

[26] Sogge, Christopher D. Lectures on non-linear wave equations. Second edition. International Press, Boston, MA, 2008. x+205 pp.

[27] Tao, Terence. Nonlinear dispersive equations. Local and global analysis. CBMS Regional Conference Series in Mathematics, 106. Published for the Conference Board of the Mathematical Sciences, Washington, DC; by the American Mathematical Society, Providence, RI, 2006. xvi+373 pp.

[28] Zhang, Junyong. Strichartz estimates and nonlinear wave equation on nontrapping asymptotically conic manifolds. Adv. Math. 271 (2015), 91-111.

Department of Mathematics, University of Chicago, 5734 S. University Avenue, Chicago, IL, 60637

E-mail address: c-rod216@math.uchicago.edu 